\title{
Radiative corrections to the neutral-current Drell-Yan process in the Standard Model and its minimal supersymmetric extension
}

\author{
Stefan Dittmaier ${ }^{a}$ and Max Huber $^{b}$ \\ ${ }^{a}$ Albert-Ludwigs-Universität Freiburg, Physikalisches Institut, \\ Hermann-Herder-Str. 3, D-79104 Freiburg, Germany \\ ${ }^{b}$ Max-Planck-Institut für Physik (Werner-Heisenberg-Institut), \\ Föhringer Ring 6, D-80805 München, Germany \\ E-mail: stefan.dittmaier@physik.uni-freiburg.de, maxh@mppmu.mpg.de
}

ABSTRACT: An adequate description of the neutral-current Drell-Yan process at the Tevatron and the LHC, in particular, requires the inclusion of electroweak radiative corrections. We extend earlier work in this direction in various ways. First, we define and numerically compare different methods to describe the Z-boson resonance including next-to-leading order electroweak corrections; moreover, we provide explicit analytical expressions for those. Second, we pay particular attention to contributions from $\gamma \gamma$ and $\gamma$-quark collisions, which involve photons in the initial state, and work out how their impact can be enhanced by selection cuts. Third, we supplement the $\mathcal{O}(\alpha)$ corrections by universal electroweak effects of higher order, such as universal two-loop contributions from $\Delta \alpha$ and $\Delta \rho$, and the leading two-loop corrections in the high-energy Sudakov regime as well as multi-photon radiation off muons in the structure-function approach. Finally, we present results on the complete next-to-leading order electroweak and QCD corrections within the minimal supersymmetric extension of the Standard Model.

KEYwORDS: Hadron-Hadron Scattering 


\section{Contents}

1 Introduction 1

2 Conventions and lowest-order cross sections 3

3 Radiative corrections to the partonic cross sections in the SM 8

3.1 Survey of radiative corrections and calculational details 9

\begin{tabular}{ll}
3.2 & Photonic and QCD corrections \\
\hline
\end{tabular}

$\begin{array}{lll}3.3 & \text { Weak corrections } & 17\end{array}$

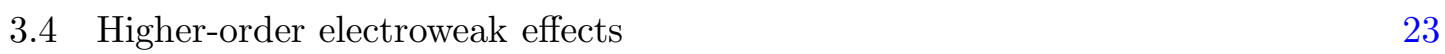

3.4.1 Leading electroweak effects and choice of couplings 23

3.4.2 Leading weak corrections in the Sudakov regime 26

$\begin{array}{lll}3.4 .3 & \text { Multi-photon final-state radiation } & 28\end{array}$

4 Radiative corrections to the partonic cross sections in the MSSM 29

5 Numerical results for the cross section $\mathrm{pp} / \mathrm{p} \overline{\mathbf{p}} \rightarrow \gamma / \mathrm{Z} \rightarrow l^{-} l^{+}+X$

5.1 Input parameters and setup for the SM 31

5.2 Phase-space cuts and event selection 31

5.3 SM predictions for cross sections at the LHC and the Tevatron 33

5.4 SM predictions for distributions at the LHC 36

5.5 Enhancing effects of incoming photons by cuts 40

5.6 Comparison to SM results of other groups 43

5.7 Numerical results on supersymmetric corrections in the MSSM 44

$\begin{array}{lll}6 & \text { Conclusions } & 49\end{array}$

$\begin{array}{ll}\text { A Factorization of the photon PDF } & 50\end{array}$

B Vertex and box corrections

C SPS benchmark scenarios $\quad 55$

\section{Introduction}

The Drell-Yan-like production of $\mathrm{W}$ and $\mathrm{Z}$ bosons both provides a standard candle for hadronic high-energy colliders as the Tevatron and the LHC and offers good possibilities to search for extra gauge bosons $\mathrm{W}^{\prime}$ and $\mathrm{Z}^{\prime}$ in high-energy tails of distributions (see, e.g., refs. $[1,2]$ and references therein). For instance, the investigation of the Z-boson resonance, which is well known from LEP and SLC experiments, is of great importance for 
detector calibration, while the analogous study of Jacobian peaks of the $\mathrm{W}$ boson in appropriate distributions even allow for precision measurements of the W-boson mass. Even the effective leptonic weak mixing angle might be measurable [3] at the LHC with a precision competing with LEP and SLC. On the theoretical side, all these tasks require precise predictions with an inclusion of both strong and electroweak radiative corrections and a careful estimate of the remaining theoretical uncertainties.

The largest corrections are due to strong interactions, mainly described by perturbative QCD. The QCD corrections are known to two loops, i.e. next-to-next-to-leading order (NNLO) for integrated cross sections [4-6] and for differential distributions [7-10]. Including corrections up to $\mathrm{N}^{3} \mathrm{LO}$ in the soft-plus-virtual approximation [11-14] the remaining theoretical error from QCD for inclusive cross sections is at the per-cent level or lower. The next-to-leading-order (NLO) QCD corrections have been matched with parton showers [15] and combined with a summation of soft gluon radiation [16-26].

While QCD corrections to on- or off-shell W- and Z-boson production with leptonic decays are very similar, electroweak corrections to the different gauge-boson production processes differ considerably. At NLO the electroweak corrections are completely known, both for charged-current (CC) [27-32] and neutral-current (NC) [33-37] processes. A tuned comparison of cross sections and differential distributions has shown good agreement between the various calculations $[1,38,39]$. Since collinear singularities from photonic initial-state radiation are absorbed into the parton distribution functions (PDF), similar to the usual QCD factorization, a photon PDF delivers another source of real electroweak corrections. Corrections due to $\gamma q$ and $\gamma \bar{q}$ collisions arise both in the CC case (W production) [40-42] and in the $\mathrm{NC}$ case (dilepton production) [36, 41]. In the $\mathrm{NC}$ case even a leading-order (LO) contribution is induced by $\gamma \gamma$ collisions [36]. Finally, the NLO calculations to the CC Drell-Yan process have been generalized to the supersymmetric extension of the Standard Model (MSSM) in ref. [42].

Beyond NLO electroweak corrections, multi-photon final-state radiation has been considered both for W-boson [42-45] and Z-boson production [46]; more recently even multiphoton radiation off all charged particles has been matched with the $\mathcal{O}(\alpha)$ corrections in the HORACE program in the CC [32] and NC [36] cases. Moreover, the impact of the leading higher-order effects due to $\Delta \alpha$ and $\Delta \rho$ as well as the leading two-loop corrections in the high-energy Sudakov regime have been investigated for the CC case in ref. [42].

A proper combination of QCD and electroweak corrections is in progress by various groups. Different procedures for this combination based on factorization or addition, as implemented in HORACE, are described in ref. [47, 48]. The results discussed there suggest that non-factorizable mixed strong-electroweak corrections, which start at the twoloop level, are required in order to achieve per-cent accuracy in the predictions. For on-shell $\mathrm{Z}$ production part of these $\mathcal{O}\left(\alpha \alpha_{\mathrm{s}}\right)$ effects have been calculated in ref. [49].

In this paper, we complete and extend the existing results on radiative corrections to the NC Drell-Yan process in various respects:

1. We rederive the $\mathcal{O}(\alpha)$ electroweak corrections and document the analytical results for the one-loop corrections explicitly. Moreover, we define and numerically compare 
different treatments of the Z-boson resonance in the presence of weak corrections. Specifically, we discuss the "complex-mass scheme" [50, 51], the "pole scheme" $[52-$ $55]$, and a scheme employing a simple factorization into the LO cross section containing the $\mathrm{Z}$ resonance and a factor for the weak correction.

2. We consistently include dilepton production processes involving photons in the initial state, which proceed via the partonic processes $\gamma \gamma \rightarrow l^{-} l^{+}, q \gamma \rightarrow l^{-} l^{+}+q$, and $\bar{q} \gamma \rightarrow l^{-} l^{+}+\bar{q}$. We even take into account the known NLO electroweak corrections [56] to the process $\gamma \gamma \rightarrow l^{-} l^{+}$, which contributes to the LO signal process.

3. Beyond NLO we consider universal two-loop contributions from $\Delta \alpha$ and $\Delta \rho$, the leading two-loop corrections in the high-energy Sudakov regime, and multi-photon radiation off muons in the structure-function approach [57-62].

4. Finally, we calculate the NLO electroweak and QCD corrections within the MSSM.

For the Standard Model (SM) the presentation in this paper widely follows refs. [31, 42], where the electroweak NLO corrections and the same type of effects beyond NLO are discussed for the CC Drell-Yan process. Similarly our discussion of the NLO corrections in the MSSM, presented here for the NC case, proceeds along the same lines as in ref. [42] for the CC case.

The paper is organized as follows. In section 2 we set up our conventions and give the lowest-order cross sections. Furthermore we describe and discuss the different treatments of the Z-boson resonance and the different input-parameter schemes considered in this paper, as far as it is necessary for the LO process. In section 3 the electroweak radiative corrections of points 1. -3 . given above as well as NLO QCD corrections are discussed. The NLO corrections within the MSSM are described in section 4. Our discussion of numerical results, which is presented in section 5, comprises integrated cross sections as well as differential distributions for the LHC and integrated results for the Tevatron. We also compare our results to results previously given in the literature and discuss how effects of incoming photons can be enhanced. Our conclusions are drawn in section 6 . In the appendix we describe the factorization of QED-like collinear singularities into the photon distribution function, give explicit expressions for the vertex and box corrections in the SM and provide details on the considered SUSY scenarios.

\section{Conventions and lowest-order cross sections}

In this section we set up our conventions for the discussion of the various partonic processes contributing to the production of a charged lepton pair. Apart from the Drell-Yan-like process $q \bar{q} \rightarrow \gamma / \mathrm{Z} \rightarrow l^{-} l^{+}$and its radiative corrections we consider the photon-induced process $\gamma \gamma \rightarrow l^{-} l^{+}$and its radiative corrections. Although the latter does not have a $\mathrm{Z}$ resonance, it is an irreducible background to $q \bar{q} \rightarrow \gamma / \mathrm{Z} \rightarrow l^{-} l^{+}$and therefore should be included. The (electroweak) NLO corrections to $\gamma \gamma \rightarrow l^{-} l^{+}$have been calculated in ref. [56], and we only briefly review some of the results given there. 

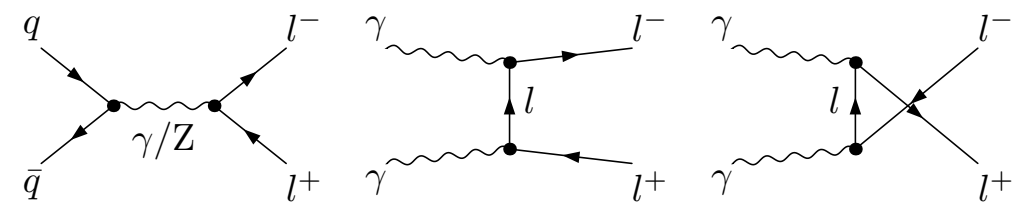

Figure 1. Partonic lowest-order diagrams for $\mathrm{pp} / \mathrm{p} \overline{\mathrm{p}} \rightarrow l^{-} l^{+}+X$.

The momenta of the incoming particles will be denoted with $p_{i}, i=1,2$, and the ones of the outgoing particles with $k_{j}, j=1,2,3$. Explicitly we assign the external momenta and helicities $\left(\sigma_{i}, \tau_{i}, \lambda\right)$ according to

$$
\begin{aligned}
q\left(p_{1}, \sigma_{1}\right)+\bar{q}\left(p_{2}, \sigma_{2}\right) & \rightarrow l^{-}\left(k_{1}, \tau_{1}\right)+l^{+}\left(k_{2}, \tau_{2}\right)\left[+\gamma / \mathrm{g}\left(k_{3}, \lambda\right)\right], \\
\gamma\left(p_{1}, \sigma_{1}\right)+\gamma\left(p_{2}, \sigma_{2}\right) & \rightarrow l^{-}\left(k_{1}, \tau_{1}\right)+l^{+}\left(k_{2}, \tau_{2}\right)\left[+\gamma\left(k_{3}, \lambda\right)\right],
\end{aligned}
$$

where $q$ generically denotes the light up- and down-type quarks, $q=\mathrm{d}, \mathrm{u}, \mathrm{s}, \mathrm{c}, \mathrm{b}$, and $l$ denotes the charged leptons $l=\mathrm{e}, \mu, \tau$. The possible photons or gluons in the final state deliver part of the real radiation contribution to the NLO corrections. The remaining part of the real NLO corrections is induced by the crossed processes $q \gamma / \mathrm{g} \rightarrow l^{-} l^{+}+q$ and $\bar{q} \gamma / \mathrm{g} \rightarrow l^{-} l^{+}+\bar{q}$. The Mandelstam variables are defined by

$$
\hat{s}=\left(p_{1}+p_{2}\right)^{2}, \quad \hat{t}=\left(p_{1}-k_{1}\right)^{2}, \quad \hat{u}=\left(p_{1}-k_{2}\right)^{2}, \quad s_{l l}=\left(k_{1}+k_{2}\right)^{2} .
$$

We neglect the fermion masses of the light quarks, $m_{q}$, and of the leptons, $m_{l}$, whenever possible, i.e. we keep these masses only as regulators in the logarithmic mass singularities originating from collinear photon emission or exchange. Obviously, we have $\hat{s}=s_{l l}$ for the non-radiative processes $q \bar{q} \rightarrow l^{-} l^{+}$and $\gamma \gamma \rightarrow l^{-} l^{+}$. At LO the Feynman diagrams shown in figure 1 contribute to the scattering amplitudes. For $q \bar{q} \rightarrow l^{-} l^{+}$the polarized Born amplitude $\mathcal{M}_{q \bar{q}}^{\mathrm{LO}}$ can be written as

$$
\mathcal{M}_{q \bar{q}}^{\mathrm{LO}, \sigma \tau}=-\frac{e^{2}}{\hat{s}} \sum_{V=\gamma, \mathrm{Z}} g_{q q V}^{\sigma} g_{l l V}^{\tau} \chi_{V}(\hat{s}) \mathcal{A}^{\sigma \tau} \equiv f_{q \bar{q}}^{\mathrm{LO}, \sigma \tau} \mathcal{A}^{\sigma \tau},
$$

where $e$ is the electric unit charge, $g_{f f V}^{\sigma}$ are the chiral couplings of the fermions $f$ to the vector bosons $V$, the functions $\chi_{V}(\hat{s})$ describe the propagation of $V$, and $\mathcal{A}^{\sigma \tau}$ are "standard matrix elements" containing the spin information of the fermions.

The standard matrix element $\mathcal{A}^{\sigma \tau}$ for the quark and lepton chiralities, $\sigma=\sigma_{1}=-\sigma_{2}$ and $\tau=\tau_{1}=-\tau_{2}$, is defined as

$$
\mathcal{A}^{\sigma \tau}=\left[\bar{v}_{q}\left(p_{2}\right) \gamma^{\mu} \omega_{\sigma} u_{q}\left(p_{1}\right)\right]\left[\bar{u}_{l}\left(k_{1}\right) \gamma_{\mu} \omega_{\tau} v_{l}\left(k_{2}\right)\right],
$$

with an obvious notation for the Dirac spinors $\bar{v}_{q}\left(p_{2}\right)$, etc., and the chirality projectors $\omega_{ \pm}=\frac{1}{2}\left(1 \pm \gamma_{5}\right)$. Explicitly the $\mathcal{A}^{\sigma \tau}$ are given by

$$
\mathcal{A}^{ \pm \pm}=2 \hat{u}, \quad \mathcal{A}^{ \pm \mp}=2 \hat{t} .
$$


For a fermion $f$ with charge $Q_{f}$ and third component $I_{\mathrm{w}, f}^{3}$ of its weak isospin the left- and right-handed couplings to $V=\gamma, \mathrm{Z}$ are given by

$$
g_{f f \gamma}^{ \pm}=-Q_{f}, \quad g_{f f Z}^{+}=-\frac{s_{\mathrm{W}}}{c_{\mathrm{W}}} Q_{f}, \quad g_{f f Z}^{-}=\frac{I_{\mathrm{W}, f}^{3}-s_{\mathrm{W}}^{2} Q_{f}}{s_{\mathrm{W}} c_{\mathrm{W}}} .
$$

The sine and cosine, $s_{\mathrm{W}}$ and $c_{\mathrm{W}}$, of the weak mixing angle are fixed by the $\mathrm{W}$ - and Z-boson masses $M_{\mathrm{W}}$ and $M_{\mathrm{Z}}$ as described below in more detail.

The propagator functions are defined by

$$
\chi_{\gamma}(\hat{s})=1, \quad \chi_{\mathrm{Z}}(\hat{s})=\frac{\hat{s}}{\hat{s}-\mu_{\mathrm{Z}}^{2}}
$$

where the complex quantities

$$
\mu_{\mathrm{Z}}^{2}=M_{\mathrm{Z}}^{2}-\mathrm{i} M_{\mathrm{Z}} \Gamma_{\mathrm{Z}}, \quad \mu_{\mathrm{W}}^{2}=M_{\mathrm{W}}^{2}-\mathrm{i} M_{\mathrm{W}} \Gamma_{\mathrm{W}}
$$

denote the locations of the poles of the Z- and W-boson propagators (with momentum transfer $p$ ) in the complex $p^{2}$ plane. The gauge-boson widths $\Gamma_{V}$ enter the propagator denominators only after performing the Dyson summation of all insertions of the (imaginary parts of the) gauge-boson self-energies, i.e. using the above $\chi_{\mathrm{Z}}(\hat{s})$ already goes beyond the lowest perturbative order. It is well known that this unavoidable mixing of perturbative orders jeopardizes the gauge invariance of predictions, in particular in the presence of radiative corrections. ${ }^{1}$ Before describing our solutions to this problem, we recall an important feature of the explicit form of the propagator function. While we have chosen a constant imaginary part in the denominator of $\chi_{\mathrm{Z}}(\hat{s})$, the frequently used on-shell (OS) renormalization scheme, as for instance defined in ref. [66], naturally leads to a running width in the denominator. In the approximation of massless decay products of the boson $V$, the OS version of $\chi_{V}(\hat{s})$ is

$$
\left.\chi_{V}(\hat{s})\right|_{\mathrm{OS}}=\frac{\hat{s}}{\hat{s}-M_{V, \mathrm{OS}}^{2}+\mathrm{i} M_{V, \mathrm{OS}} \Gamma_{V, \mathrm{OS}} \times \hat{s} / M_{V, \mathrm{OS}}^{2} \times \theta(\hat{s})} .
$$

The two versions of $\chi_{V}(\hat{s})$ are formally equivalent in the resonance region if mass and width of $V$ are properly translated $[67,68]$

$$
M_{V}=\left.\frac{M_{V}}{\sqrt{1+\Gamma_{V}^{2} / M_{V}^{2}}}\right|_{\mathrm{OS}}, \quad \Gamma_{V}=\left.\frac{\Gamma_{V}}{\sqrt{1+\Gamma_{V}^{2} / M_{V}^{2}}}\right|_{\mathrm{OS}} .
$$

Since the $\mathrm{W}$ and $\mathrm{Z}$ masses and widths are usually quoted in the OS scheme, we shall perform this translation before our evaluations. For the masses, the impact of this conversion typically is $M_{\mathrm{Z}, \mathrm{OS}}-M_{\mathrm{Z}} \approx 34 \mathrm{MeV}$ and $M_{\mathrm{W}, \mathrm{OS}}-M_{\mathrm{W}} \approx 27 \mathrm{MeV}$. We perform our evaluation in the following schemes for treating the Z-boson resonance, where at this point we describe the various procedures only as far as relevant in LO and give the details for the corrections in the next section:

\footnotetext{
${ }^{1}$ More details on this issue, specific examples as well as proposed solutions can, e.g., be found in refs. [63$65]$ and references therein.
} 
- Complex-mass scheme (CMS): The CMS was introduced in ref. [50] for LO calculations and generalized to NLO in ref. [51]. In this approach the squared W-and Z-boson masses are consistently identified with $\mu_{\mathrm{W}}^{2}$ and $\mu_{\mathrm{Z}}^{2}$, respectively, i.e. with the location of the poles of the propagators in the complex $p^{2}$ plane. This leads to complex couplings and, in particular, a complex weak mixing angle via $c_{\mathrm{W}}^{2}=1-s_{\mathrm{W}}^{2}=\mu_{\mathrm{W}}^{2} / \mu_{\mathrm{Z}}^{2}$. The scheme fully respects all relations that follow from gauge invariance (Ward or Slavnov-Taylor identities, gauge-parameter cancellation), because the gauge-boson masses are modified only by an analytic continuation. Beyond LO the complex masses are introduced directly at the level of the Lagrangian by splitting the real bare masses into complex renormalized masses and complex counterterms, so that the usual perturbative calculus with Feynman rules and counterterms works without modification. In contrast to gauge invariance, unitarity is not respected order by order in perturbation theory. However, spurious terms spoiling unitarity are of (N)NLO in an (N)LO calculation without any unnatural amplification, because unitarity cancellations, which are ruled by gauge invariance, are respected. More details of this scheme can also be found in ref. [63].

In the CMS the LO amplitude (2.4) is, thus, evaluated with complex couplings $g_{f f V}^{ \pm}$ and a complex Z-boson mass.

- Pole scheme (PS): The PS exploits the fact that both the location $\mu_{V}^{2}$ of the $V$ propagator pole and its residue in amplitudes are gauge-independent quantities [52, 69-73]. The idea [52-55] is, thus, to first isolate the residue for the considered resonance and subsequently to introduce a finite decay width only in the gauge-independent resonant part. If done carefully this procedure respects gauge invariance, but it should be kept in mind that the resonant part of an amplitude is not uniquely determined by the propagator structure alone, but depends on a specific phase-space parameterization and in most cases also on the separation of polarization-dependent parts. A "pole approximation" - in contrast to a full PS calculation as performed in this paper - results from a resonant amplitude defined in the PS upon neglecting non-resonant parts.

The LO amplitude (2.4) with real couplings defined via the usual on-shell relation $c_{\mathrm{W}}^{2}=1-s_{\mathrm{W}}^{2}=M_{\mathrm{W}}^{2} / M_{\mathrm{Z}}^{2}$, but with the complex Z-boson mass in $\chi_{\mathrm{Z}}(\hat{s})$, represents the result of a particular PS variant. The PS operation here first splits off the polarization-dependent structure $\mathcal{A}^{\sigma \tau}$ and subsequently introduces the Z-boson width in the resonant part of the form factors via $1 /\left(\hat{s}-M_{\mathrm{Z}}^{2}\right) \rightarrow 1 /\left(\hat{s}-M_{\mathrm{Z}}^{2}+\mathrm{i} M_{\mathrm{Z}} \Gamma_{\mathrm{Z}}\right)=$ $\chi_{\mathrm{Z}}(\hat{s}) / \hat{s}$, while the non-resonant photon part is not changed.

- Factorization scheme (FS): Many variants of factorizing resonant structures from amplitudes have been suggested and used in the literature, but they all share the idea to separate a simple resonant factor from a potentially complicated amplitude that does not involve resonances anymore. In ref. [31], for instance, the virtual electroweak correction to Drell-Yan-like W production was factorized from the resonant LO amplitude, so that the relative correction factor did not involve resonance factors anymore. ${ }^{2}$

\footnotetext{
${ }^{2}$ The relative electroweak correction defined in this way involves the W-boson width $\Gamma_{\mathrm{W}}$ only in loga-
} 
For the present case of NC dilepton production we start from the LO amplitude (2.4) with real couplings, as in the PS, and define the relative correction factor for the weak (i.e. non-photonic) one-loop correction in the strict limit of vanishing gauge-boson widths.

We can, thus, compare two different versions of LO cross sections for $q \bar{q} \rightarrow \gamma / \mathrm{Z} \rightarrow l^{-} l^{+}$: one version delivered by the CMS, another by the PS and FS, which coincide in LO.

The electromagnetic coupling $\alpha=e^{2} /(4 \pi)$ yields an overall factor to the LO predictions. Although the electric charge is always defined (renormalized) in the Thomson limit, the value for $\alpha$ can be fixed in different input-parameter schemes. We support the following three different schemes:

- $\alpha(0)$-scheme: The fine-structure constant $\alpha(0)$ and all particle masses define the complete input. In this scheme, the relative corrections to the $q \bar{q} \rightarrow \gamma / \mathrm{Z} \rightarrow l^{-} l^{+}$ cross sections sensitively depend on the light-quark masses via $\alpha \ln m_{q}$ terms that enter the charge renormalization.

- $\alpha\left(M_{\mathrm{Z}}\right)$-scheme: The effective electromagnetic coupling $\alpha\left(M_{\mathrm{Z}}\right)$ and all particle masses define the basic input. Tree-level couplings are derived from $\alpha\left(M_{\mathrm{Z}}\right)$, and the relative corrections receive contributions from the quantity $\Delta \alpha\left(M_{\mathrm{Z}}\right)$, which accounts for the running of the electromagnetic coupling from scale $Q=0$ to $Q=M_{\mathrm{Z}}$ (induced by light fermions) and cancels the corresponding $\alpha \ln m_{q}$ terms that appear in the corrections to the $q \bar{q}$ channels in the $\alpha(0)$-scheme.

- $G_{\mu}$-scheme: The Fermi constant $G_{\mu}$ and all particle masses define the basic input. Tree-level couplings are derived from the effective coupling $\alpha_{G_{\mu}}=\sqrt{2} G_{\mu} M_{\mathrm{W}}^{2}(1-$ $\left.M_{\mathrm{W}}^{2} / M_{\mathrm{Z}}^{2}\right) / \pi$, and the relative corrections receive contributions from the quantity $\Delta r$ [74-76], which describes the radiative corrections to muon decay. Since $\Delta \alpha\left(M_{\mathrm{Z}}\right)$ is contained in $\Delta r$, there is no large effect on the $q \bar{q}$ channels induced by the running of the electromagnetic coupling in the $G_{\mu}$-scheme either.

Since light-quark masses are perturbatively ill-defined and can only play the role of phenomenological fit parameters, the $\alpha\left(M_{\mathrm{Z}}\right)$ - and $G_{\mu}$-schemes are preferable over the $\alpha(0)$ scheme for the $q \bar{q}$ annihilation processes. More details on the difference of the three schemes are provided in the next section, where we deal with electroweak radiative corrections (see also ref. [31]).

The differential LO cross section $\mathrm{d} \hat{\sigma}_{q \bar{q}}^{\mathrm{LO}} / \mathrm{d} \hat{\Omega}$ is easily obtained by squaring the LO matrix element $\mathcal{M}_{q \bar{q}}^{\mathrm{LO}}$,

$$
\begin{aligned}
& \left(\frac{\mathrm{d} \hat{\sigma}_{q \overline{\hat{q}}}^{\mathrm{LO}}}{\mathrm{d} \hat{\Omega}}\right)=\frac{1}{12} \frac{1}{64 \pi^{2} \hat{s}} \sum_{\mathrm{pol}}\left|\mathcal{M}_{q \bar{q}}^{\mathrm{LO}}\right|^{2}=\frac{\alpha^{2}}{12 \hat{s}^{3}}\left\{2 Q_{q}^{2} Q_{l}^{2}\left(\hat{t}^{2}+\hat{u}^{2}\right)\right. \\
& \quad+2 Q_{q} Q_{l} \operatorname{Re}\left[\left[\left(g_{q q Z}^{+} g_{l l Z}^{+}+g_{q q Z}^{-} g_{l l Z}^{-}\right) \hat{u}^{2}+\left(g_{q q Z}^{+} g_{l l Z}^{-}+g_{q q Z}^{-} g_{l l Z}^{+}\right) \hat{t}^{2}\right] \chi_{\mathrm{Z}}(\hat{s})\right]
\end{aligned}
$$

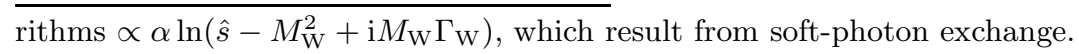



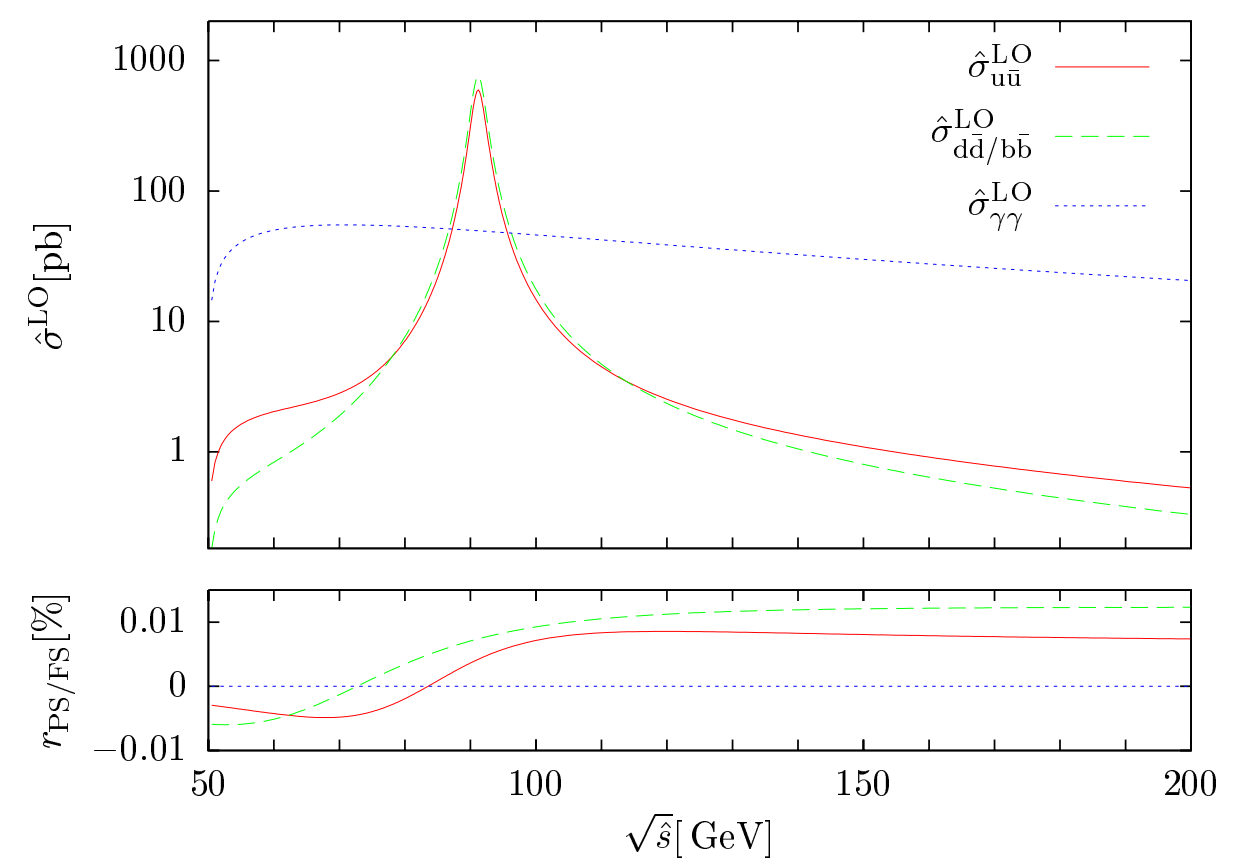

Figure 2. LO cross sections for $u \bar{u} / d \bar{d} \rightarrow \gamma / \mathrm{Z} \rightarrow l^{-} l^{+}$in the vicinity of the $\mathrm{Z}$ resonance using the different schemes (CMS and PS/FS) for treating finite-width effects, employing the $G_{\mu}$-scheme, and the LO cross section for $\gamma \gamma \rightarrow l^{-} l^{+}$.

$$
\left.+\left[\left(\left|g_{q q Z}^{+}\right|^{2}\left|g_{l l Z}^{+}\right|^{2}+\left|g_{q q Z}^{-}\right|^{2}\left|g_{l l Z}^{-}\right|^{2}\right) \hat{u}^{2}+\left(\left|g_{q q Z}^{+}\right|^{2}\left|g_{l l Z}^{-}\right|^{2}+\left|g_{q q Z}^{-}\right|^{2}\left|g_{l l Z}^{+}\right|^{2}\right) \hat{t}^{2}\right]\left|\chi_{Z}(\hat{s})\right|^{2}\right\}
$$

The explicit factor $1 / 12$ results from the average over the quark spins and colours, and $\hat{\Omega}$ is the solid angle of the outgoing $l^{-}$in the partonic centre-of-mass frame. In figure 2 we show the integrated partonic LO cross sections $\hat{\sigma}_{q \bar{q}}^{\mathrm{LO}}(\hat{s})$ for the different schemes (CMS and PS/FS) to treat the finite $\mathrm{Z}$ width, as obtained in the $G_{\mu}$-scheme. We also show the relative difference $r_{\mathrm{PS} / \mathrm{FS}}=\left.\hat{\sigma}^{\mathrm{LO}}\right|_{\mathrm{PS} / \mathrm{FS}} /\left.\hat{\sigma}^{\mathrm{LO}}\right|_{\mathrm{CMS}}-1$ of the results obtained in the different schemes, which turns out to be at the 0.01 per-cent level.

For completeness we state the contribution of $\gamma \gamma \rightarrow l^{-} l^{+}$, d $\hat{\sigma}_{\gamma \gamma}^{\mathrm{LO}} / \mathrm{d} \hat{\Omega}$, to the LO differential cross section,

$$
\left(\frac{\mathrm{d} \hat{\sigma}_{\gamma \gamma}^{\mathrm{LO}}}{\mathrm{d} \hat{\Omega}}\right)=\frac{1}{4} \frac{1}{64 \pi^{2} \hat{s}} \sum_{\text {pol }}\left|\mathcal{M}_{\gamma \gamma}^{\mathrm{LO}}\right|^{2}=\frac{\alpha^{2}}{2 \hat{s}}\left(\frac{\hat{t}}{\hat{u}}+\frac{\hat{u}}{\hat{t}}\right) .
$$

For details we refer to ref. [56]. Here we just mention that we consider $\gamma \gamma \rightarrow l^{-} l^{+}$cross sections always in the $\alpha(0)$-scheme, because the natural scale for the coupling of the external photons is $Q=0$. In fact, using the $\alpha\left(M_{\mathrm{Z}}\right)$ - or $G_{\mu}$-scheme here would result in large corrections containing $\alpha \ln m_{q}$ terms, which should be avoided.

\section{Radiative corrections to the partonic cross sections in the SM}

In this section we discuss the NLO radiative corrections to the partonic subprocesses contributing to the hadronic process $\mathrm{pp} / \mathrm{p} \overline{\mathrm{p}} \rightarrow l^{-} l^{+}+X$. For the main contribution of $q \bar{q}$ 
annihilation, many issues discussed here are very similar to the case of $\mathrm{e}^{+} \mathrm{e}^{-} \rightarrow \gamma / \mathrm{Z} \rightarrow f \bar{f}$ as measured in the LEP and SLD experiments, for which precision calculations have been performed in the last two decades (see, e.g., refs. [77, 78] and references therein).

\subsection{Survey of radiative corrections and calculational details}

The electroweak radiative NLO corrections can be divided into photonic and weak corrections. The photonic corrections consist of real and virtual corrections that are induced by the emission and exchange of an additional photon. Since only electrically neutral gauge bosons are involved at LO, the photonic subset of the complete $\mathcal{O}(\alpha)$ electroweak corrections is separately invariant under $\mathrm{U}(1)_{\text {elmg }}$ gauge transformations. For the $q \bar{q}$ channel this classification is, e.g., discussed in ref. [65] in more detail, for the $\gamma \gamma$ channel this separation was introduced in ref. [56]. For $q \bar{q}$ annihilation the photonic corrections can be further classified into separately U(1)elmg gauge-invariant parts. Specifically, the photonic contributions can be split into initial-state corrections, final-state corrections, and interference terms, according to their charge proportionality to $Q_{q}^{2}, Q_{l}^{2}$, and $Q_{q} Q_{l}$, respectively. In this sense the photonic corrections to the $\gamma \gamma$ channel are final-state corrections proportional to $Q_{l}^{2}$. The virtual photonic corrections to the $q \bar{q}$ channel are composed of the one-loop photon exchange diagrams shown in figure 3a) and the corresponding counterterm contributions; the counterparts for $\gamma \gamma$ scattering can be found in ref. [56]. The real photonic corrections consist of processes with single-photon emission, $q \bar{q} \rightarrow \gamma / \mathrm{Z} \rightarrow l^{-} l^{+}+\gamma$ and $\gamma \gamma \rightarrow l^{-} l^{+}+\gamma$, and of the processes $q / \bar{q} \gamma \rightarrow \gamma / \mathrm{Z} \rightarrow l^{-} l^{+}+q / \bar{q}$, which deliver a correction to both LO processes $q \bar{q} \rightarrow l^{-} l^{+}$and $\gamma \gamma \rightarrow l^{-} l^{+}$. On the hadronic level the photon-induced processes are, of course, suppressed due to the smallness of the photon PDF, but on the partonic level all processes are of the same order $\mathcal{O}(\alpha)$ compared to the LO processes. Since real photons effectively couple with $\alpha(0)$ and since virtual and real photonic corrections are intrinsically linked to each other, it is natural to identify the relative coupling of the whole photonic correction with $\alpha(0)$, independent of the choice of the input-parameter scheme chosen in LO. This means in $q \bar{q}$ annihilation (and in the crossing-related $q / \bar{q} \gamma$ scattering) and in the $\gamma \gamma$ channel we scale the cross section contributions of the photonic corrections with $\alpha(0) \alpha^{2}$ and $\alpha(0)^{3}$, respectively, where $\alpha$ depends on the input-parameter scheme as discussed in section 2 .

The weak $\mathcal{O}(\alpha)$ corrections to the $q \bar{q}$ channel comprise contributions of the transverse parts of the photon, the $\mathrm{Z}$, and the $\gamma \mathrm{Z}$ mixing self-energies $\left(\Sigma_{\mathrm{T}}^{\gamma \gamma}, \Sigma_{\mathrm{T}}^{\mathrm{ZZ}}\right.$, and $\left.\Sigma_{\mathrm{T}}^{\gamma \mathrm{Z}}\right)$, of weak corrections to the $\gamma / \mathrm{Z} \bar{q} q$ and $\gamma / \mathrm{Z} l^{-} l^{+}$vertices, the $\mathrm{ZZ}$ and WW box diagrams, and counterterms. The diagrams for the vertex and box corrections are shown in figure $3 \mathrm{~b}$ ) for incoming quarks other than b's. For incoming b-quarks, the same diagrams as for incoming $\mathrm{d}$ - or s-quarks exist, but in diagrams with internal $\mathrm{W}$ bosons the b-quark turns into its massive iso-spin partner, the top-quark. For this reason, in 't Hooft-Feynman gauge there are additional versions of those diagrams in which one or two $\mathrm{W}$ bosons are replaced by would-be Goldstone bosons; these diagrams are shown in figure 3c). Details and explicit results on the weak corrections to the $\gamma \gamma$ channel can be found in ref. [56]. In our explicit evaluation we scale the relative weak correction with the coupling $\alpha$ as defined 
a)
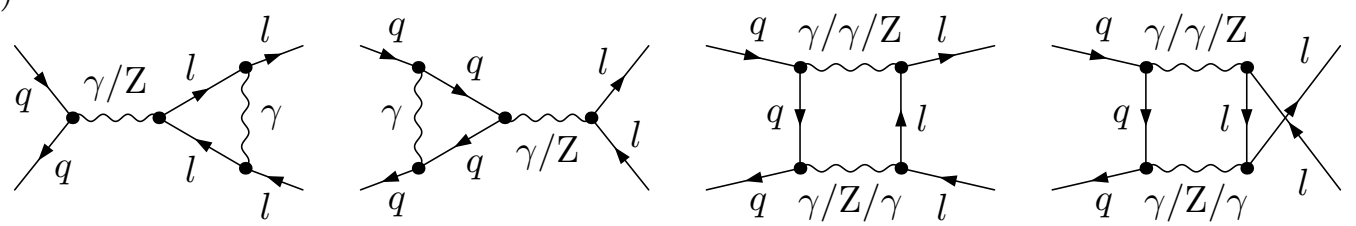

b)
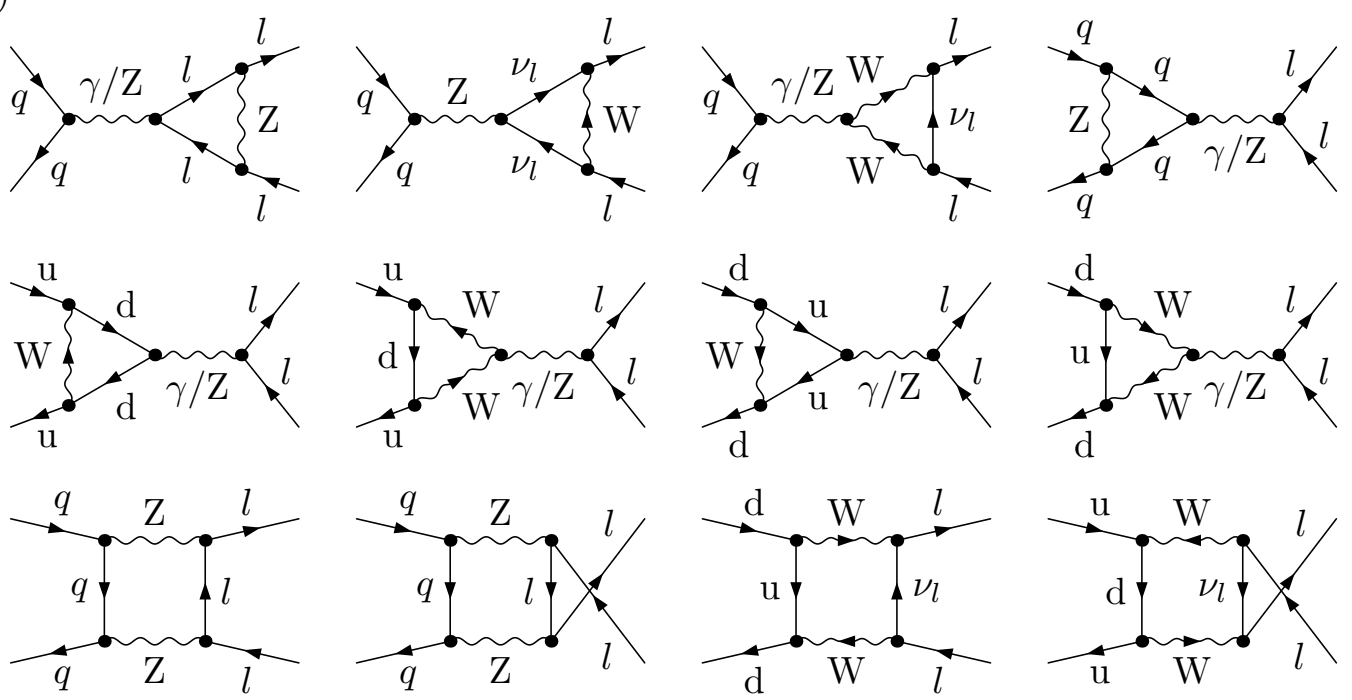

c)
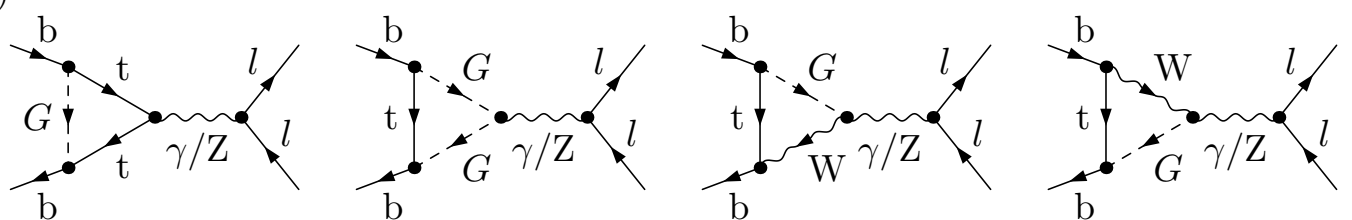

Figure 3. Vertex and box diagrams for the electroweak virtual corrections to $q \bar{q} \rightarrow l^{-} l^{+}$: a) photonic corrections, b) weak corrections with light incoming quarks $q=\mathrm{u}, \mathrm{d}, \mathrm{c}, \mathrm{s}, \mathrm{b}$, and c) additional diagrams for incoming b-quarks, where $G$ stands for would-be Goldstone boson fields.

in the respective input-parameter scheme, i.e. the cross section contributions of the weak corrections scale like $\alpha^{3}$ and $\alpha \alpha(0)^{2}$ in the $q \bar{q}$ and $\gamma \gamma$ channels, respectively.

The NLO QCD corrections to $q \bar{q} \rightarrow l^{-} l^{+}$are easily obtained from the photonic initialstate corrections, i.e. by setting the lepton charge $Q_{l}$ to zero within the photonic corrections, and replacing $\alpha(0) Q_{q}^{2} \rightarrow \alpha_{\mathrm{s}}\left(\mu_{\mathrm{R}}\right) C_{\mathrm{F}}$, with $C_{\mathrm{F}}=4 / 3$ and $\alpha_{\mathrm{s}}\left(\mu_{\mathrm{R}}\right)$ representing the strong coupling constant at renormalization scale $\mu_{\mathrm{R}}$. For squared amplitudes with an incoming gluon, $q / \bar{q} \mathrm{~g} \rightarrow \gamma / \mathrm{Z} \rightarrow l^{-} l^{+}+q / \bar{q}$, we omit diagrams with the external photon coupling to $l$ and replace $\alpha(0) Q_{q}^{2} \rightarrow \alpha_{\mathrm{s}}\left(\mu_{\mathrm{R}}\right) T_{\mathrm{F}} / 3$ with $T_{\mathrm{F}}=1 / 2$ in the respective squared amplitudes with an incoming photon instead of a gluon.

For this work we have rederived the NLO corrections to the $q \bar{q}$ channel with stan- 
dard methods. More precisely, we performed two independent calculations, with results in mutual agreement. The one-loop diagrams and amplitudes are generated with FEYNARTS versions $1.0[79,80]$ and 3.2 [81]. The subsequent algebraic reductions to standard forms are done with inhouse MATHEMATICA routines in one version and with the help of FORMCALC $[82,83]$ and FEYNCALC $[84,85]$ in the other. In this reduction the appearing tensor integrals are reduced to scalar integrals with the Passarino-Veltman algorithm [86]. The scalar integrals are evaluated using the methods and results of ref. [87-89], where UV divergences are treated in dimensional regularization and the soft and collinear singularities are regularized by small fermion masses and an infinitesimal photon or gluon mass $m_{\gamma / g}$. Since the application of the CMS requires complex gauge-boson masses, the results of refs. [87-89] on the loop integrals had to be generalized accordingly. ${ }^{3}$ The amplitude of the virtual correction, $\mathcal{M}_{q \bar{q}}^{\text {virt, } \sigma \tau}$, can be expressed in terms of a "form factor" $f_{q \bar{q}}^{\mathrm{virt}, \sigma \tau}=f_{q \bar{q}, \text { phot }}^{\mathrm{virt}, \sigma \tau}+f_{q \bar{q} \text {, weak }}^{\mathrm{virt}, \sigma \tau}+f_{q \bar{q}, \mathrm{QCD}}^{\mathrm{virt}, \sigma \tau}$ times the LO Dirac structure $\mathcal{A}^{\sigma \tau}$,

$$
\mathcal{M}_{q \bar{q}}^{\mathrm{virt}, \sigma \tau}=\left(f_{q \bar{q}, \mathrm{phot}}^{\mathrm{virt}, \sigma \tau}+f_{q \bar{q}, \text { weak }}^{\mathrm{virt}, \sigma \tau}+f_{q \bar{q}, \mathrm{QCD}}^{\mathrm{virt}, \sigma \tau}\right) \mathcal{A}^{\sigma \tau} .
$$

For self-energy and vertex corrections this is obviously possible, since all external fermions are considered in the massless limit. In $D \neq 4$ space-time dimensions the calculation of box diagrams actually leads to combinations of Dirac chains that are not present at LO. However, since the box diagrams are UV finite the four-dimensionality of space-time can be used to reduce all Dirac structures to the one of $\mathcal{M}_{q \bar{q}}^{\mathrm{LO}}$, as explained in appendix B in more detail. Finally, we have rederived the photonic and QCD corrections for massless external fermions, photons, and gluons within dimensional regularization by making use of the results of ref. [92] for translating the IR-divergent scalar integrals from mass into dimensional regularization and by employing the statements made in the appendix of ref. [93] on the structure of rational terms of IR origin. The results of mass and dimensional regularization for IR divergences are in perfect agreement.

Details of our calculation of real photonic (or gluonic) corrections are provided in the following section, where we present our results on the photonic and QCD corrections. The contributions resulting from the factorization of mass-singular initial-state photonic or gluonic corrections are also reviewed there.

We conclude this overview by summarizing the structure of the hadronic cross section including the full NLO corrections,

$$
\begin{gathered}
\sigma_{h_{1} h_{2}}^{\mathrm{NLO}}\left(P_{1}, P_{2}\right)=\int_{0}^{1} \mathrm{~d} x_{1} \int_{0}^{1} \mathrm{~d} x_{2}\left\{\sum_{q=\mathrm{u}, \mathrm{d}, \mathrm{c}, \mathrm{s}, \mathrm{b}} f_{q}^{\left(h_{1}\right)}\left(x_{1}, \mu_{\mathrm{F}}^{2}\right) f_{\bar{q}}^{\left(h_{2}\right)}\left(x_{2}, \mu_{\mathrm{F}}^{2}\right)\right. \\
\times\left[\int \mathrm{d} \hat{\sigma}_{q \bar{q}}^{\mathrm{LO}}\left(x_{1} P_{1}, x_{2} P_{2}\right)\left(1+\delta_{q \bar{q}, \mathrm{QCD}}^{\mathrm{virt}}+\delta_{q \bar{q}, \mathrm{phot}}^{\mathrm{virt}}+\delta_{q \bar{q}, \text { weak }}^{\mathrm{virt}}\right)\right. \\
+\int \mathrm{d} \hat{\sigma}_{q \bar{q}, \mathrm{QCD}}^{\mathrm{real}}\left(x_{1} P_{1}, x_{2} P_{2}\right)+\int \mathrm{d} \hat{\sigma}_{q \bar{q}, \mathrm{QCD}}^{\mathrm{fact}}\left(x_{1} P_{1}, x_{2} P_{2}\right)
\end{gathered}
$$

\footnotetext{
${ }^{3}$ In detail, the scalar two- and three-point functions with complex masses can be explicitly found in refs. [90] and [87], respectively. The IR-divergent four-point integral of ref. [88] is also valid for an internal complex mass, so that only the regular four-point functions, as e.g. given in ref. [89] for real mass parameters, had to be analytically continued to complex masses. General results on singular and regular four-point integrals with complex masses will be published elsewhere [91].
} 


$$
\begin{aligned}
& \left.+\int \mathrm{d} \hat{\sigma}_{q \bar{q}, \mathrm{phot}}^{\mathrm{real}}\left(x_{1} P_{1}, x_{2} P_{2}\right)+\int \mathrm{d} \hat{\sigma}_{q \bar{q}, \mathrm{phot}}^{\mathrm{fact}}\left(x_{1} P_{1}, x_{2} P_{2}\right)\right] \\
& +\sum_{q=\mathrm{u}, \mathrm{d}, \mathrm{c}, \mathrm{s}, \mathrm{b}} f_{\mathrm{g}}^{\left(h_{1}\right)}\left(x_{1}, \mu_{\mathrm{F}}^{2}\right)\left[f_{q}^{\left(h_{2}\right)}\left(x_{2}, \mu_{\mathrm{F}}^{2}\right)\left(\int \mathrm{d} \hat{\sigma}_{\mathrm{gq}}^{\mathrm{LO}}\left(x_{1} P_{1}, x_{2} P_{2}\right)+\int \mathrm{d} \hat{\sigma}_{\mathrm{gq}}^{\mathrm{fact}}\left(x_{1} P_{1}, x_{2} P_{2}\right)\right)\right. \\
& \quad+(q \rightarrow \bar{q})] \\
& +\sum_{q=\mathrm{u}, \mathrm{d}, \mathrm{c}, \mathrm{s}, \mathrm{b}} f_{\gamma}^{\left(h_{1}\right)}\left(x_{1}, \mu_{\mathrm{F}}^{2}\right)\left[f_{q}^{\left(h_{2}\right)}\left(x_{2}, \mu_{\mathrm{F}}^{2}\right)\left(\int \mathrm{d} \hat{\sigma}_{\gamma q}^{\mathrm{LO}}\left(x_{1} P_{1}, x_{2} P_{2}\right)+\int \mathrm{d} \hat{\sigma}_{\gamma q}^{\text {fact }}\left(x_{1} P_{1}, x_{2} P_{2}\right)\right)\right. \\
& \quad+(q \rightarrow \bar{q})] \\
& +\frac{1}{2} f_{\gamma}^{\left(h_{1}\right)}\left(x_{1}, \mu_{\mathrm{F}}^{2}\right) f_{\gamma}^{\left(h_{2}\right)}\left(x_{2}, \mu_{\mathrm{F}}^{2}\right)\left[\int \mathrm{d} \hat{\sigma}_{\gamma \gamma}^{\mathrm{LO}}\left(x_{1} P_{1}, x_{2} P_{2}\right)\left(1+\delta_{\gamma \gamma, \mathrm{phot}}^{\text {virt }}+\delta_{\gamma \gamma, \text { weak }}^{\text {virt }}\right)\right. \\
& \left.\left.\quad+\int \mathrm{d} \hat{\sigma}_{\gamma \gamma, \mathrm{phot}}^{\mathrm{real}}\left(x_{1} P_{1}, x_{2} P_{2}\right)+\int \mathrm{d} \hat{\sigma}_{\gamma \gamma, \mathrm{phot}}^{\mathrm{fact}}\left(x_{1} P_{1}, x_{2} P_{2}\right)\right]\right\} \\
& +\left(h_{1} \leftrightarrow h_{2}\right) .
\end{aligned}
$$

Here $f_{a}^{\left(h_{i}\right)}\left(x, \mu_{\mathrm{F}}^{2}\right)$ are the NLO PDF for finding the parton $a$ with momentum fraction $x$ in the hadron $h_{i}$ with momentum $P_{i}$ at the factorization scale $\mu_{\mathrm{F}}$. The contributions $\hat{\sigma}_{a b, \mathrm{QCD} / \mathrm{phot}}^{\mathrm{fact}}$ result from the PDF redefinitions that describe the absorption of collinear initial-state singularities of gluonic or photonic origin (see next section). The factors $\delta_{a b, X}^{\mathrm{virt}}$ represent the virtual corrections to the squared LO matrix elements for the $a b$ initial state,

$$
2 \operatorname{Re}\left\{\mathcal{M}_{a b, X}^{\mathrm{virt}}\left(\mathcal{M}_{a b, X}^{\mathrm{LO}}\right)^{*}\right\} \equiv \delta_{a b, X}^{\mathrm{virt}}\left|\mathcal{M}_{a b, X}^{\mathrm{LO}}\right|^{2} .
$$

\subsection{Photonic and QCD corrections}

The issue of a gauge-invariant treatment of the photonic and QCD corrections has been discussed in ref. [65] in detail (including even massive fermions). From the arguments given there and the discussion above, it is clear that a consistent way of evaluating the photonic and QCD corrections is to use the complex Z-boson mass $\mu_{\mathrm{Z}}$ wherever it appears. Since the weak mixing angle is derived from the ratio of the $\mathrm{W}$ and $\mathrm{Z}$ masses, and $M_{\mathrm{W}}$ does not appear elsewhere in these corrections, the quantity $c_{\mathrm{W}}$ can be treated as free parameter in the context of photonic and QCD corrections, and $s_{\mathrm{W}}$ as well as the couplings $g_{f f Z}^{ \pm}$are derived from $c_{\mathrm{W}}$. Specifically, we set $c_{\mathrm{W}}$ to $\mu_{\mathrm{W}} / \mu_{\mathrm{Z}}$ in the CMS and to $M_{\mathrm{W}} / M_{\mathrm{Z}}$ in the PS and FS; the numerical difference is, however, marginal, as expected.

The virtual photonic corrections can be decomposed into vertex and box contributions,

$$
f_{q \bar{q}, \text { phot }}^{\mathrm{virt}, \sigma \tau}=f_{q \bar{q}, \text { phot }}^{\mathrm{vert}, \sigma \tau}(\hat{s})+f_{q \bar{q}, \text { phot }}^{\mathrm{box}, \sigma \tau}(\hat{s}, \hat{t})
$$

where the vertex part contains also the photonic contributions to the fermionic wavefunction corrections. The vertex correction $f_{\text {phot }}^{\text {vert }}(\hat{s})$ consists of an initial- and a final-state part and reads

$$
f_{q \bar{q}, \text { phot }}^{\mathrm{vert}, \sigma \tau}(\hat{s})=-\frac{e^{2}}{\hat{s}}\left[\hat{F}_{q q V, \text { phot }}(\hat{s})+\hat{F}_{l l V, \text { phot }}(\hat{s})\right]\left[Q_{q} Q_{l}+g_{q q Z}^{\sigma} g_{l l Z}^{\tau} \chi_{\mathrm{Z}}(\hat{s})\right]
$$



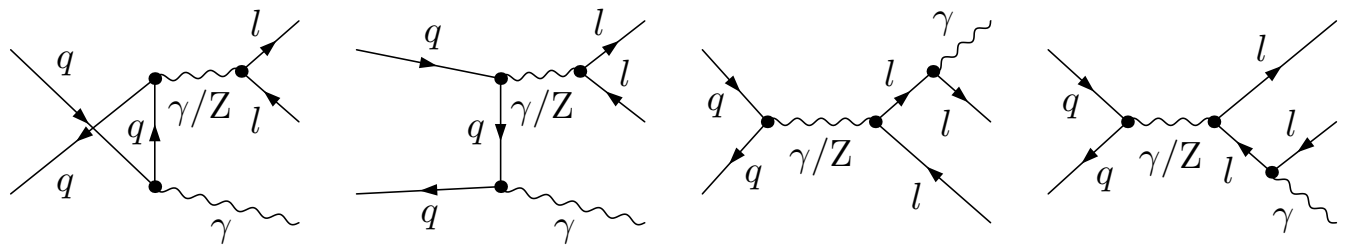

Figure 4. Diagrams for real-photon emission.
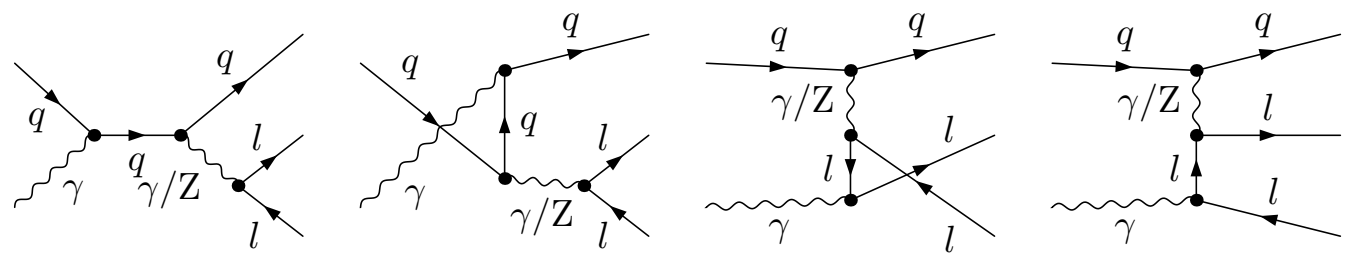

Figure 5. Diagrams for photon-induced processes with incoming quarks.

with the renormalized vertex form factor

$\hat{F}_{f f V, \operatorname{phot}}(\hat{s})=-\frac{Q_{f}^{2} \alpha}{2 \pi}\left[\ln \left(\frac{m_{\gamma}^{2}}{\hat{s}}\right) \ln \left(\frac{m_{f}^{2}}{\hat{s}}\right)+\ln \left(\frac{m_{\gamma}^{2}}{\hat{s}}\right)+\frac{1}{2} \ln \left(\frac{m_{f}^{2}}{\hat{s}}\right)-\frac{1}{2} \ln ^{2}\left(\frac{m_{f}^{2}}{\hat{s}}\right)-\frac{2 \pi^{2}}{3}+2\right]$,

where irrelevant imaginary parts have been discarded. The interference terms of the virtual photonic corrections are due to the photonic box diagrams and can be written as

$$
f_{q \bar{q}, \text { phot }}^{\text {box, } \sigma \tau}(\hat{s}, \hat{t})=f_{q \bar{q}}^{\gamma \gamma, \sigma \tau}(\hat{s}, \hat{t})+f_{q \bar{q}}^{\mathrm{Z} \gamma, \sigma \tau}(\hat{s}, \hat{t}) \text {. }
$$

The correction factors $f_{q \bar{q}}^{V V^{\prime}, \sigma \tau}$ are given in appendix B.

The real photonic bremsstrahlung corrections to $q \bar{q} \rightarrow \gamma / \mathrm{Z} \rightarrow l^{-} l^{+}$, whose diagrams are shown in figures 4 and 5, are calculated using the Weyl-van-der-Waerden spinor formalism adopting the conventions of ref. [94]. This results in very compact expressions for the helicity amplitudes $\mathcal{M}_{a b}^{\sigma_{1}, \sigma_{2}, \tau_{1}, \tau_{2}}(\lambda)=\sum_{V=\gamma, \mathrm{Z}} \mathcal{M}_{a b, V}^{\sigma_{1}, \sigma_{2}, \tau_{1}, \tau_{2}}(\lambda)$, where $a b=q \bar{q}, q \gamma, \bar{q} \gamma$ refers to the partonic initial states and $V$ to the exchanged bosons in the respective diagrams. For real-photon emission we get

$$
\begin{aligned}
& \mathcal{M}_{q \bar{q}, V}^{-++-}(+)=2 \sqrt{2} e^{3} g_{q q V}^{-} g_{l l V}^{+}\left\langle p_{2} k_{2}\right\rangle^{2}\left[\frac{Q_{q}}{s_{l l}-\mu_{V}^{2}} \frac{\left\langle k_{1} k_{2}\right\rangle^{*}}{\left\langle p_{1} k_{3}\right\rangle\left\langle p_{2} k_{3}\right\rangle}-\frac{Q_{l}}{\hat{s}-\mu_{V}^{2}} \frac{\left\langle p_{1} p_{2}\right\rangle^{*}}{\left\langle k_{1} k_{3}\right\rangle\left\langle k_{2} k_{3}\right\rangle}\right], \\
& \mathcal{M}_{q \bar{q}, V}^{-+++}(+)=-2 \sqrt{2} e^{3} g_{q q V}^{-} g_{l l V}^{-}\left\langle p_{2} k_{1}\right\rangle^{2}\left[\frac{Q_{q}}{s_{l l}-\mu_{V}^{2}} \frac{\left\langle k_{1} k_{2}\right\rangle^{*}}{\left\langle p_{1} k_{3}\right\rangle\left\langle p_{2} k_{3}\right\rangle}-\frac{Q_{l}}{\hat{s}-\mu_{V}^{2}} \frac{\left\langle p_{1} p_{2}\right\rangle^{*}}{\left\langle k_{1} k_{3}\right\rangle\left\langle k_{2} k_{3}\right\rangle}\right]
\end{aligned}
$$

in the limit of massless fermions, and we have defined $\mu_{\gamma}=0$. The spinor products are defined by

$$
\langle p q\rangle=\epsilon^{A B} p_{A} q_{B}=2 \sqrt{p_{0} q_{0}}\left[\mathrm{e}^{-\mathrm{i} \phi_{p}} \cos \frac{\theta_{p}}{2} \sin \frac{\theta_{q}}{2}-\mathrm{e}^{-\mathrm{i} \phi_{q}} \cos \frac{\theta_{q}}{2} \sin \frac{\theta_{p}}{2}\right],
$$


where $p_{A}, q_{A}$ are the associated momentum spinors for the light-like momenta

$$
\begin{aligned}
p^{\mu} & =p_{0}\left(1, \sin \theta_{p} \cos \phi_{p}, \sin \theta_{p} \sin \phi_{p}, \cos \theta_{p}\right), \\
q^{\mu} & =q_{0}\left(1, \sin \theta_{q} \cos \phi_{q}, \sin \theta_{q} \sin \phi_{q}, \cos \theta_{q}\right) .
\end{aligned}
$$

Owing to helicity conservation in the case of massless fermions all amplitudes with $\sigma_{1}=\sigma_{2}$ or $\tau_{1}=\tau_{2}$ vanish. The remaining six non-zero helicity amplitudes are obtained from the amplitudes (3.8) via a parity $(\mathrm{P})$ transformation

$$
\mathcal{M}_{a b, V}^{-\sigma_{1},-\sigma_{2},-\tau_{1},-\tau_{2}}(-\lambda)=\left.\operatorname{sgn}\left(\sigma_{1} \sigma_{2} \tau_{1} \tau_{2}\right)\left(\mathcal{M}_{a b, V}^{\sigma_{1}, \sigma_{2}, \tau_{1}, \tau_{2}}(\lambda)\right)^{*}\right|_{g_{f f V}^{ \pm} \leftrightarrow g_{f f V}^{\mp}},
$$

and a CP transformation

$$
\mathcal{M}_{a b, V}^{-\sigma_{2},-\sigma_{1},-\tau_{2},-\tau_{1}}(-\lambda)=-\left.\operatorname{sgn}\left(\sigma_{1} \sigma_{2} \tau_{1} \tau_{2}\right)\left(\mathcal{M}_{a b, V}^{\sigma_{1}, \sigma_{2}, \tau_{1}, \tau_{2}}(\lambda)\right)^{*}\right|_{\substack{p_{1} \leftrightarrow p_{2} \\ k_{1} \leftrightarrow k_{2}}}
$$

Note that in the above formulas the complex masses in the propagators and the couplings are not complex conjugated, since $\mathrm{P}$ and $\mathrm{CP}$ transformations only act on the wave functions and momenta entering the amplitudes.

Apart from the partonic channels with a $q \bar{q}$ pair in the initial state, we also include the photon-induced processes whose diagrams are shown in figure 5 for incoming quarks. Of course, there are also the corresponding channels for incoming anti-quarks. The amplitudes for the photon-induced processes

$$
\begin{aligned}
& q\left(p_{1}, \sigma_{1}\right)+\gamma\left(p_{2}, \lambda\right) \rightarrow l^{-}\left(k_{1}, \tau_{1}\right)+l^{+}\left(k_{2}, \tau_{2}\right)+q\left(k_{3}, \sigma_{1}\right), \\
& \gamma\left(p_{1}, \lambda\right)+\bar{q}\left(p_{2}, \sigma_{2}\right) \rightarrow l^{-}\left(k_{1}, \tau_{1}\right)+l^{+}\left(k_{2}, \tau_{2}\right)+\bar{q}\left(k_{3}, \sigma_{2}\right)
\end{aligned}
$$

are related to the ones for real-photon emission by crossing symmetry,

$$
\begin{aligned}
& \mathcal{M}_{q \gamma}^{\sigma_{1},-\sigma_{2}, \tau_{1}, \tau_{2}}(-\lambda)=-\left.\operatorname{sgn}\left(\sigma_{2}\right) \mathcal{M}_{q \bar{q}}^{\sigma_{1}, \sigma_{2}, \tau_{1}, \tau_{2}}(\lambda)\right|_{p_{2} \leftrightarrow-k_{3}}, \\
& \mathcal{M}_{\gamma \bar{q}}^{-\sigma_{1}, \sigma_{2}, \tau_{1}, \tau_{2}}(-\lambda)=-\left.\operatorname{sgn}\left(\sigma_{1}\right) \mathcal{M}_{q \bar{q}}^{\sigma_{1}, \sigma_{2}, \tau_{1}, \tau_{2}}(\lambda)\right|_{p_{1} \leftrightarrow-k_{3}} .
\end{aligned}
$$

In terms of Weyl-van-der-Waerden spinors the crossing transformation $p \rightarrow-p$ of a fourmomentum $p$ is obtained by inverting the conjugated parts only,

$$
p_{\dot{A}} \rightarrow-p_{\dot{A}}, \quad p_{A} \rightarrow p_{A} .
$$

The contributions $\hat{\sigma}_{q \bar{q}}^{\text {real }}$ and $\hat{\sigma}_{q / \bar{q} \gamma}^{\text {real }}$ to the partonic cross section are given by

$$
\int \mathrm{d} \hat{\sigma}_{a b}^{\text {real }}=\frac{N_{\mathrm{c}, a b}}{4} \frac{1}{2 \hat{s}} \int \mathrm{d} \Phi_{\gamma} \sum_{\mathrm{pol}}\left|\mathcal{M}_{a b}^{\sigma_{1}, \sigma_{2}, \tau_{1}, \tau_{2}}(\lambda)\right|^{2},
$$

where the colour factors for the different initial states are $N_{\mathrm{c}, q \bar{q}}=1 / 3$ and $N_{\mathrm{c}, q \gamma}=N_{\mathrm{c}, \bar{q} \gamma}=$ 1. The phase-space integral is defined by

$$
\int \mathrm{d} \Phi_{\gamma}=\int \frac{\mathrm{d}^{3} \mathbf{k}_{1}}{(2 \pi)^{3} 2 k_{1,0}} \int \frac{\mathrm{d}^{3} \mathbf{k}_{2}}{(2 \pi)^{3} 2 k_{2,0}} \int \frac{\mathrm{d}^{3} \mathbf{k}_{3}}{(2 \pi)^{3} 2 k_{3,0}}(2 \pi)^{4} \delta\left(p_{1}+p_{2}-k_{1}-k_{2}-k_{3}\right) .
$$


The phase-space integrals in the real corrections $\hat{\sigma}_{q \bar{q}}^{\text {real }}$ and $\hat{\sigma}_{q / \bar{q} \gamma}^{\text {real contain logarithmic }}$ collinear divergences in the limit of massless fermions. Moreover, the real-photon emission integral contains a logarithmic soft singularity because of the masslessness of the photon. To regularize the soft and collinear singularities we introduce small fermion masses and an infinitesimal photon mass according to the generally known factorization properties of the squared amplitudes in the singular phase-space regions. This step is usually performed via phase-space slicing, which isolates singular regions in phase space, or via a subtraction formalism, which employs an auxiliary function in the whole phase space in order to cancel all singularities. In our calculation we proceed as in the treatment of hadronic $\mathrm{W}$ production as described in refs. [31, 42], i.e. we employ three different methods:

(i) soft phase-space slicing with effective collinear factors [31],

(ii) two-cutoff phase-space slicing [27] for soft and collinear singularities, and

(iii) dipole subtraction $[95,96]$.

Since the detailed formulas for the CC case can be transferred to the present NC case in a straightforward way, we do not go into formal details here, but restrict ourselves to the most important features of the singularity structure in the final result.

The analytical results on the photonic corrections to $\gamma \gamma \rightarrow l^{-} l^{+}$can be found in ref. [56]. The following discussion of final- and initial-state singularities includes both $q \bar{q}$ and $\gamma \gamma$ scattering.

Two types of final-state collinear singularities arise. First, there is a collinear singularity if the $l^{-} l^{+}$system in the final state receives a small invariant mass $M_{l l}$, e.g., via a collinear $\gamma^{*} \rightarrow l^{-} l^{+}$splitting. Since we, however, set a lower limit on $M_{l l}$, this singular configuration is excluded from our region of interest. Second, collinear photon radiation off the final-state charged leptons is enhanced by the mass-singular factor $\alpha \ln \left(Q / m_{l}\right)$ (with $Q$ denoting a typical hard scale). The nature of this singularity is discussed in more detail in section 3.4.3, where an effective treatment of collinear multi-photon emission is described.

Singularities connected to collinear splittings in the initial state result from $q \rightarrow \mathrm{g} / \gamma q^{*}$, $\bar{q} \rightarrow \mathrm{g} / \gamma \bar{q}^{*}$ (gluon/photon bremsstrahlung in $q \bar{q}$ annihilation), $\mathrm{g} / \gamma \rightarrow q \bar{q}^{*}, \mathrm{~g} / \gamma \rightarrow \bar{q} q^{*}$ (gluon/photon splittings into $q \bar{q}$ pairs in $\mathrm{g} / \gamma q$ and $\mathrm{g} / \gamma \bar{q}$ scattering), $q \rightarrow q \gamma^{*}, \bar{q} \rightarrow \bar{q} \gamma^{*}$ (forward scattering of $q$ or $\bar{q}$ in $\gamma q / \bar{q}$ scattering), and $\gamma \rightarrow l^{ \pm} l^{\mp *}$ (photon splitting into $l^{+} l^{-}$ pairs in $\gamma q / \bar{q}$ and $\gamma \gamma$ scattering). The last splitting corresponds to configurations with a charged lepton $l^{ \pm}$lost in the beam direction (proton remnant), i.e. it only contributes if not both charged leptons are required in the event signature; the contribution of this configuration is enhanced by the factor $\alpha \ln \left(Q / m_{l}\right)$ (again with $Q$ denoting a typical hard scale). The other splittings lead to $l^{+} l^{-}$pairs in the final state with a gluon, (anti-)quark, or photon lost in the proton remnant; the corresponding contributions are enhanced by factors $\alpha_{\mathrm{s}} \ln \left(Q / m_{q}\right)$ and $\alpha Q_{q}^{2} \ln \left(Q / m_{q}\right)$ for gluonic and photonic splittings, respectively. These (non-perturbative) singular contributions are absorbed into the PDF via factorization, where finite contributions to this PDF redefinition define the factorization scheme. In detail the LO PDF $f_{a}^{(h)}(x)$, describing the emission of parton $a$ out of the hadron $h$ with 
longitudinal momentum fraction $x$, are split according to (see, e.g., ref. [27, 97])

$$
\begin{aligned}
f_{q / \bar{q}}^{(h)}(x) \rightarrow f_{q / \bar{q}}^{(h)}\left(x, \mu_{\mathrm{F}}^{2}\right)- & \frac{\alpha_{\mathrm{s}} C_{\mathrm{F}}}{2 \pi} \int_{x}^{1} \frac{\mathrm{d} z}{z} f_{q / \bar{q}}^{(h)}\left(\frac{x}{z}, \mu_{\mathrm{F}}^{2}\right) \\
& \quad \times\left\{\ln \left(\frac{\mu_{\mathrm{F}}^{2}}{m_{q}^{2}}\right)\left[P_{f f}(z)\right]_{+}-\left[P_{f f}(z)(2 \ln (1-z)+1)\right]_{+}+C_{f f}^{\overline{\mathrm{MS}}}(z)\right\} \\
- & \frac{\alpha_{\mathrm{s}} T_{\mathrm{F}}}{2 \pi} \int_{x}^{1} \frac{\mathrm{d} z}{z} f_{g}^{(h)}\left(\frac{x}{z}, \mu_{\mathrm{F}}^{2}\right)\left\{\ln \left(\frac{\mu_{\mathrm{F}}^{2}}{m_{q}^{2}}\right) P_{f \gamma}(z)+C_{f \gamma}^{\overline{\mathrm{MS}}}(z)\right\} \\
- & \frac{\alpha Q_{q}^{2}}{2 \pi} \int_{x}^{1} \frac{\mathrm{d} z}{z} f_{q / \bar{q}}^{(h)}\left(\frac{x}{z}, \mu_{\mathrm{F}}^{2}\right) \\
& \times\left\{\ln \left(\frac{\mu_{\mathrm{F}}^{2}}{m_{q}^{2}}\right)\left[P_{f f}(z)\right]_{+}-\left[P_{f f}(z)(2 \ln (1-z)+1)\right]_{+}+C_{f f}^{\mathrm{DIS}}(z)\right\} \\
- & 3 \frac{\alpha Q_{q}^{2}}{2 \pi} \int_{x}^{1} \frac{\mathrm{d} z}{z} f_{\gamma}^{(h)}\left(\frac{x}{z}, \mu_{\mathrm{F}}^{2}\right)\left\{\ln \left(\frac{\mu_{\mathrm{F}}^{2}}{m_{q}^{2}}\right) P_{f \gamma}(z)+C_{f \gamma}^{\mathrm{DIS}}(z)\right\}, \\
f_{\gamma}^{(h)}(x) \rightarrow f_{\gamma}^{(h)}\left(x, \mu_{\mathrm{F}}^{2}\right)- & \frac{\alpha Q_{q}^{2}}{2 \pi} \sum_{a=q, \bar{q}} \int_{x}^{1} \frac{\mathrm{d} z}{z} f_{a}^{(h)}\left(\frac{x}{z}, \mu_{\mathrm{F}}^{2}\right) \\
& \times\left\{\ln \left(\frac{\mu_{\mathrm{F}}^{2}}{m_{q}^{2}}\right) P_{\gamma f}(z)-P_{\gamma f}(z)(2 \ln z+1)+C_{\gamma f}^{\mathrm{DIS}}(z)\right\}
\end{aligned}
$$

into NLO PDF $f_{a}^{(h)}\left(x, \mu_{\mathrm{F}}^{2}\right)$, which now include parton emission up to a scale of the order of the factorization scale $\mu_{\mathrm{F}}$. The splitting functions are given by

$$
P_{f f}(z)=\frac{1+z^{2}}{1-z}, \quad P_{f \gamma}(z)=z^{2}+(1-z)^{2}, \quad P_{\gamma f}(z)=\frac{1+(1-z)^{2}}{z}
$$

The coefficient functions $C_{i j}(z)$, defining the finite parts, coincide with the usual definition in $D$-dimensional regularization for exactly massless partons where the $\ln m_{q}$ terms appear as $1 /(D-4)$ poles. Details about the photon PDF redefinition are given in appendix A. Following standard definitions of QCD, we distinguish the $\overline{\mathrm{MS}}$ and DIS-like schemes which are formally defined by the coefficient functions

$$
\begin{aligned}
C_{f f}^{\overline{\mathrm{MS}}} & =C_{f \gamma}^{\overline{\mathrm{MS}}}=C_{\gamma f}^{\overline{\mathrm{MS}}}=0, \\
C_{f f}^{\mathrm{DIS}}(z) & =\left[P_{f f}(z)\left(\ln \left(\frac{1-z}{z}\right)-\frac{3}{4}\right)+\frac{9+5 z}{4}\right]_{+}, \\
C_{f \gamma}^{\mathrm{DIS}}(z) & =P_{f \gamma}(z) \ln \left(\frac{1-z}{z}\right)-8 z^{2}+8 z-1, \\
C_{\gamma f}^{\mathrm{DIS}}(z) & =-C_{f f}^{\mathrm{DIS}}(z) .
\end{aligned}
$$

We use the MRST2004qed PDF [98] which consistently include $\mathcal{O}\left(\alpha_{\mathrm{s}}\right)$ QCD and $\mathcal{O}(\alpha)$ photonic corrections. These PDF include a photon distribution function for the proton and thus allow to take into account photon-induced partonic processes. As explained 
in ref. [97], the consistent use of these PDF requires the $\overline{\mathrm{MS}}$ factorization scheme for the QCD, but the DIS scheme for the photonic corrections. ${ }^{4}$

The contributions $\hat{\sigma}_{a b, \mathrm{QCD}}^{\mathrm{fact}}$ phot appearing in (3.2) result from the corrections in the PDF replacements (3.19) and (3.20) after these substitutions are made in the LO prediction for the hadronic cross section. More precisely, $\hat{\sigma}_{a b, \mathrm{QCD} / \text { phot }}^{\mathrm{fact}}$ corresponds to the NLO $\mathrm{QCD} /$ photonic contribution (i.e. linearized in $\alpha_{\mathrm{s}}$ or $\alpha$ ) proportional to the PDF combination $f_{a}^{\left(h_{1}\right)}\left(x_{1}, \mu_{\mathrm{F}}^{2}\right) f_{b}^{\left(h_{2}\right)}\left(x_{2}, \mu_{\mathrm{F}}^{2}\right)$ of the partonic $a b$ initial state.

\subsection{Weak corrections}

In the following we sketch the structure of the weak corrections and emphasize those points that are relevant for the treatment of the resonance and for the change from one input-parameter scheme to another. The correction factor $f_{q \bar{q}, \text { weak }}^{\mathrm{virt}}$, which is introduced in eq. (3.1), is decomposed according to the splitting into self-energy, vertex, and box diagrams,

$$
f_{q \bar{q}, \text { weak }}^{\text {virt, } \sigma \tau}=f_{q \bar{q}, \text { weak }}^{\text {self }, \sigma \tau}(\hat{s})+f_{q \bar{q}, \text { weak }}^{\text {vert, } \sigma \tau}(\hat{s})+f_{q \bar{q}, \text { weak }}^{\text {box }, \sigma \tau}(\hat{s}, \hat{t}) .
$$

The self-energy corrections comprise contributions from the $\gamma \gamma, \gamma \mathrm{Z}$, and ZZ self-energies, the results of which can be found in ref. [66] in 't Hooft-Feynman gauge. Self-energy corrections to the external fermion states are absorbed into vertex counterterms, as usually done in on-shell renormalization schemes. The one-loop diagrams for the weak vertex and box corrections are shown in figures $3 \mathrm{~b}$ ) and c), their complete expressions are provided in appendix B.

(i) Complex-mass scheme.

We first describe the calculation in the CMS. The self-energy corrections explicitly read

$$
f_{q \bar{q}, \text { weak }}^{\text {self }, \sigma \tau}(\hat{s})=e^{2} \frac{Q_{q} Q_{l}}{\hat{s}^{2}} \hat{\Sigma}_{\mathrm{T}}^{\gamma \gamma}(\hat{s})+e^{2} \frac{g_{q q Z}^{\sigma} g_{l l Z}^{\tau}}{\left(\hat{s}-\mu_{\mathrm{Z}}^{2}\right)^{2}} \hat{\Sigma}_{\mathrm{T}}^{\mathrm{ZZ}}(\hat{s})-e^{2} \frac{Q_{l} g_{q q Z}^{\sigma}+Q_{q} g_{l l Z}^{\tau}}{\hat{s}\left(\hat{s}-\mu_{\mathrm{Z}}^{2}\right)} \hat{\Sigma}_{\mathrm{T}}^{\gamma \mathrm{Z}}(\hat{s}),
$$

where $\hat{\Sigma}_{\mathrm{T}}^{V V^{\prime}}$ denote the renormalized (transverse) gauge-boson self-energies,

$$
\begin{aligned}
& \Sigma_{\mathrm{T}}^{\gamma \gamma}(\hat{s})=\Sigma_{\mathrm{T}}^{\gamma \gamma}(\hat{s})+\delta \mathcal{Z}_{\gamma \gamma} \hat{s}, \\
& \Sigma_{\mathrm{T}}^{\mathrm{ZZ}}(\hat{s})=\Sigma_{\mathrm{T}}^{\mathrm{ZZ}}(\hat{s})-\delta M_{\mathrm{Z}}^{2}+\delta \mathcal{Z}_{\mathrm{ZZ}}\left(\hat{s}-\mu_{\mathrm{Z}}^{2}\right), \\
& \Sigma_{\mathrm{T}}^{\gamma \mathrm{Z}}(\hat{s})=\Sigma_{\mathrm{T}}^{\gamma \mathrm{Z}}(\hat{s})+\frac{1}{2} \delta \mathcal{Z}_{\gamma \mathrm{Z}} \hat{s}+\frac{1}{2} \delta \mathcal{Z}_{\mathrm{Z} \gamma}\left(\hat{s}-\mu_{\mathrm{Z}}^{2}\right) .
\end{aligned}
$$

As mentioned above, the explicit results of ref. [66] on the unrenormalized self-energies $\Sigma_{\mathrm{T}}^{V V^{\prime}}$ can be used, however, we stress that complex gauge-boson masses and couplings

\footnotetext{
${ }^{4}$ Note that our choice of the factorization scheme and coefficient functions for incoming photons differs from the previously presented results [36, 41]. In ref. [41] the $\overline{\mathrm{MS}}$ scheme was employed, and the coefficient function $C_{\gamma f}^{\text {DIS }}(z)$ of ref. [36] was fixed somewhat ad hoc. Our redefined photon PDF is fixed in such a way that the momentum sum rule for the total proton momentum is respected, i.e. our fixation of the photon PDF follows the same logic as the gluon PDF redefinition in the DIS scheme for QCD factorization. In the first preprint version of this paper we employed a different factorization prescription that also respected the proton momentum sum rule, but was not in line with the standard NLO QCD conventions for factorization schemes. Therefore, we switched to the scheme described here (see comments at the end of appendix A).
} 
have to be inserted everywhere. The renormalization constants $\delta M_{\mathrm{Z}}^{2}$ and $\delta \mathcal{Z}_{V V^{\prime}}$ are defined in eqs. (4.9) and (4.10) of ref. [51] for the CMS. They are expressed in terms of gauge-boson self-energies and consistently evaluated with complex parameters (but real-valued momenta); in particular, no real part is taken in their definition, in contrast to the usual on-shell renormalization scheme, as, e.g., defined in ref. [66].

The vertex corrections can be written as

$$
\begin{aligned}
f_{q \bar{q}, \text { weak }}^{\mathrm{vert}, \sigma \tau}(\hat{s})= & -e^{2} \frac{Q_{q} Q_{l}}{\hat{s}}\left[\hat{F}_{q q \gamma, \text { weak }}^{\sigma}(\hat{s})+\hat{F}_{l l \gamma, \text { weak }}^{\tau}(\hat{s})\right] \\
& -e^{2} \frac{g_{q q Z}^{\sigma} g_{l l Z}^{\tau}}{\hat{s}-\mu_{\mathrm{Z}}^{2}}\left[\hat{F}_{q q Z, \text { weak }}^{\sigma}(\hat{s})+\hat{F}_{l l Z, \text { weak }}^{\tau}(\hat{s})\right],
\end{aligned}
$$

with the renormalized vertex form factors

$$
\hat{F}_{f f V \text {,weak }}^{\sigma}(\hat{s})=F_{f f V \text {,weak }}^{\sigma}(\hat{s})+\delta_{f f V \text {,weak }}^{\text {ct, } \sigma} .
$$

The explicit expressions for the unrenormalized form factors $F_{f f V \text {,weak }}(\hat{s})$ are given in appendix B. The subscript "weak" indicates that the contributions from photon-exchange diagrams are omitted both in the form factors and in the vertex counterterms $\delta_{f f V \text {, weak }}^{\text {ct, } \sigma}$. In the $\alpha(0)$-scheme, the counterterms are given by

$$
\begin{aligned}
& \delta_{f f \gamma, \text { weak }}^{\mathrm{ct}, \sigma}=\frac{\delta e}{e}+\frac{1}{2} \delta \mathcal{Z}_{\gamma \gamma}+\delta \mathcal{Z}_{f, \text { weak }}^{\sigma}-\frac{1}{2} \frac{g_{f f Z}^{\sigma}}{Q_{f}} \delta \mathcal{Z}_{\mathrm{Z} \gamma}, \\
& \delta_{f f Z, \text { weak }}^{\mathrm{ct}, \sigma}=\frac{\delta g_{f f Z}^{\sigma}}{g_{f f Z}^{\sigma}}+\frac{1}{2} \delta \mathcal{Z}_{\mathrm{ZZ}}+\delta \mathcal{Z}_{f, \text { weak }}^{\sigma}-\frac{1}{2} \frac{Q_{f}}{g_{f f Z}^{\sigma}} \delta \mathcal{Z}_{\gamma \mathrm{Z}},
\end{aligned}
$$

with

$$
\begin{aligned}
& \delta g_{f f Z}^{+}=-\frac{s_{\mathrm{W}}}{c_{\mathrm{W}}} Q_{f}\left(\frac{\delta e}{e}+\frac{1}{c_{\mathrm{W}}^{2}} \frac{\delta s_{\mathrm{W}}}{s_{\mathrm{W}}}\right), \\
& \delta g_{f f Z}^{-}=\frac{I_{\mathrm{w}, f}^{3}}{s_{\mathrm{W}} c_{\mathrm{W}}}\left(\frac{\delta e}{e}+\frac{s_{\mathrm{W}}^{2}-c_{\mathrm{W}}^{2}}{c_{\mathrm{W}}^{2}} \frac{\delta s_{\mathrm{W}}}{s_{\mathrm{W}}}\right)+\delta g_{f f Z}^{+} .
\end{aligned}
$$

Note that the subscript "weak" appears only on the fermionic wave-function renormalization constants $\delta \mathcal{Z}_{f}^{\sigma}$, obtained from the the fermion self-energies, because only those receive a photonic contribution. We again emphasize the difference between the renormalization constants in the CMS [51] and the usual on-shell scheme [66]. In the CMS, all quantities are derived from complex masses and couplings, and no real parts are taken from the self-energies that enter the renormalization constants. Explicit results can be found in ref. [51]. In particular, the renormalization constant of the weak mixing angle, $\delta s_{\mathrm{W}}$, is connected to the mass renormalization of the complex gauge-boson masses.

The charge renormalization constant $\delta e / e$ contains logarithms of the light-fermion masses, inducing large corrections proportional to $\alpha \ln m_{f}$, which are related to the running of the electromagnetic coupling $\alpha(Q)$ from $Q=0$ to a high-energy scale. In order to render these quark-mass logarithms meaningful, it is necessary to adjust 
these masses to the asymptotic tail of the hadronic contribution to the vacuum polarization $\Pi^{\gamma \gamma}\left(Q^{2}\right)=\Sigma_{\mathrm{T}}^{\gamma \gamma}\left(Q^{2}\right) / Q^{2}$ of the photon. Using $\alpha\left(M_{\mathrm{Z}}\right)$, as defined in ref. [99], as input this adjustment is implicitly incorporated, and the counterterm reads

$$
\left.\delta_{f f V}^{\mathrm{ct}, \sigma}\right|_{\alpha\left(M_{\mathrm{Z}}\right)}=\left.\delta_{f f V}^{\mathrm{ct}, \sigma}\right|_{\alpha(0)}-\frac{1}{2} \Delta \alpha\left(M_{\mathrm{Z}}\right)
$$

where

$$
\Delta \alpha\left(M_{\mathrm{Z}}\right)=\Pi_{f \neq \mathrm{t}}^{\gamma \gamma}(0)-\operatorname{Re}\left\{\Pi_{f \neq \mathrm{t}}^{\gamma \gamma}\left(M_{\mathrm{Z}}^{2}\right)\right\} \approx \frac{\alpha(0)}{3 \pi} \sum_{f \neq \mathrm{t}} N_{f}^{\mathrm{c}} Q_{f}^{2}\left[\ln \left(\frac{M_{\mathrm{Z}}^{2}}{m_{f}^{2}}\right)-\frac{5}{3}\right],
$$

with $\Pi_{f \neq t}^{\gamma \gamma}$ denoting the photonic vacuum polarization induced by all fermions other than the top quark (see also ref. [66]), and $N_{l}^{\mathrm{c}}=1$ and $N_{q}^{\mathrm{c}}=3$ are the colour factors for leptons and quarks, respectively. In contrast to the $\alpha(0)$-scheme the counterterm $\left.\delta_{f f V}^{\text {ct, } \sigma}\right|_{\alpha\left(M_{\mathrm{z}}\right)}$ does not involve light-quark masses, since all corrections of the form $\alpha^{n} \ln ^{n}\left(m_{f}^{2} / M_{\mathrm{Z}}^{2}\right)$ are absorbed in the LO cross section parametrized by $\alpha\left(M_{\mathrm{Z}}\right)=\alpha(0) /\left[1-\Delta \alpha\left(M_{\mathrm{Z}}\right)\right]$. In the $G_{\mu}$-scheme, the transition from $\alpha(0)$ to $G_{\mu}$ is ruled by the quantity $\Delta r^{(1)}$, which is deduced from muon decay,

$$
\alpha_{G_{\mu}} \equiv \frac{\sqrt{2} G_{\mu} M_{\mathrm{W}}^{2}\left(M_{\mathrm{Z}}^{2}-M_{\mathrm{W}}^{2}\right)}{\pi M_{\mathrm{Z}}^{2}}=\alpha(0)\left(1+\Delta r^{(1)}\right)+\mathcal{O}\left(\alpha^{3}\right) .
$$

The counterterm $\delta_{f f V}^{\mathrm{ct}, \sigma}$ in the $G_{\mu}$-scheme reads

$$
\left.\delta_{f f V}^{\mathrm{ct}, \sigma}\right|_{G_{\mu}}=\left.\delta_{f f V}^{\mathrm{ct}, \sigma}\right|_{\alpha(0)}-\frac{1}{2} \Delta r^{(1)}
$$

where the one-loop correction $\Delta r^{(1)}$ is evaluated with complex masses and couplings in the CMS. This translation of $\Delta r^{(1)}$ into the CMS is easily obtained upon analytical continuation of the result given in ref. [66] in the on-shell scheme. Note that $\Delta r^{(1)}$ implicitly contains large contributions from $\Delta \alpha\left(M_{\mathrm{Z}}\right) \sim 6 \%$ and the (one-loop) correction $\left(c_{\mathrm{W}}^{2} / s_{\mathrm{W}}^{2}\right) \Delta \rho^{(1)} \sim 3 \%$ induced by the $\rho$-parameter, where $\Delta \rho^{(1)} \propto G_{\mu} m_{\mathrm{t}}^{2}$. Thus, the large fermion-mass logarithms are also resummed in the $G_{\mu}$-scheme, and the LO cross section in $G_{\mu}$-parametrization absorbs large universal corrections induced by the $\rho$-parameter. In section 3.4.1 we further elaborate on higher-order effects induced by $\Delta \alpha$ and $\Delta \rho$.

The box correction $f_{q \bar{q}}^{\text {box, } \sigma \tau}(\hat{s}, \hat{t})$ is the only virtual correction that depends also on the scattering angle, i.e. on the variables $\hat{t}$ and $\hat{u}=-\hat{s}-\hat{t}$. The boxes are decomposed into the contributions of the $\mathrm{ZZ}$ and $\mathrm{WW}$ box diagrams,

$$
f_{q \bar{q}, \text { weak }}^{\mathrm{box}, \sigma \tau}(\hat{s}, \hat{t})=f_{q \bar{q}}^{\mathrm{ZZ}, \sigma \tau}(\hat{s}, \hat{t})+f_{q \bar{q}}^{\mathrm{WW}, \sigma \tau}(\hat{s}, \hat{t}) .
$$

The individual correction factors are given in appendix B. In appendix B we also give the explicit expressions for the vertex and box corrections for incoming b-quarks, where due to the large mass of the top quark additional diagrams [see figure 3c)] have to be taken into account. 
(ii) Pole scheme.

As explained in section 2, the application of the pole scheme [52-55] starts from a fixed-order calculation without any special treatment of the resonance. Specifically we calculate the weak corrections in the on-shell renormalization scheme of ref. [66], i.e. in our PS calculation all masses and couplings are real quantities and the $\mathrm{Z}$ decay width $\Gamma_{Z}$ only appears where it is made explicit in the following formulas. The input-parameter schemes are defined as in the previous section, with $\Delta \alpha$ and $\Delta r^{(1)}$ derived from real input parameters.

In a second step, the resonance pole is isolated from the non-resonant remainder and dressed by a properly Dyson-summed Breit-Wigner propagator. The definition of a gauge-independent residue on resonance, in general, involves some freedom in the more-dimensional phase space, because the resonance location fixes only a single invariant. In our case, for instance, two different definitions of the residue result if we write the resonant contribution to the LO amplitude either as $r_{1}(\hat{s}, \hat{t}) /\left(\hat{s}-M_{\mathrm{Z}}^{2}\right)$ or as $r_{2}(\hat{s}, \hat{u}) /\left(\hat{s}-M_{\mathrm{Z}}^{2}\right)$ and simply set $\hat{s} \rightarrow M_{\mathrm{Z}}^{2}$ in the numerators. Off resonance $\left(\hat{s} \neq M_{\mathrm{Z}}^{2}\right)$ the two versions for the residue, $r_{1}\left(M_{\mathrm{Z}}^{2}, \hat{t}\right)$ and $r_{2}\left(M_{\mathrm{Z}}^{2}, \hat{u}\right)$, obviously are not the same due to $\hat{s}+\hat{t}+\hat{u}=0$. We apply the pole scheme to the form factors $f_{q \bar{q} \text {,weak }}^{\mathrm{virt}, \sigma \tau}$ as defined in (3.1), i.e. we single out the resonance pole after splitting off the spin-dependent standard matrix elements $\mathcal{A}^{\sigma \tau}$ from the amplitude. Note that the resonant part of $f_{q \bar{q}, \text { weak }}^{\text {virt }, \tau \tau}$ comprises self-energy and vertex corrections only, which merely depend on $\hat{s}$, but not on the variables $\hat{t}$ and $\hat{u}$.

For the vertex corrections this procedure is very simple. The contributions involving Z-boson exchange, $f_{q \bar{q}}^{\text {vert,Z, } \sigma \tau}$, are modified as follows,

$$
\begin{aligned}
f_{q \bar{q}, \text { weak }}^{\mathrm{vert}, \sigma \tau}(\hat{s})= & -e^{2} \frac{g_{q q Z}^{\sigma} g_{l l Z}^{\tau}}{\hat{s}-M_{\mathrm{Z}}^{2}}\left[\hat{F}_{q q Z, \text { weak }}^{\sigma}(\hat{s})+\hat{F}_{l l Z, \text { weak }}^{\tau}(\hat{s})\right] \\
\rightarrow & -e^{2} g_{q q Z}^{\sigma} g_{l l Z}^{\tau}\left[\frac{\hat{F}_{q q Z \text {,weak }}^{\sigma}\left(M_{\mathrm{Z}}^{2}\right)+\hat{F}_{l l Z, \text { weak }}^{\tau}\left(M_{\mathrm{Z}}^{2}\right)}{\hat{s}-M_{\mathrm{Z}}^{2}+\mathrm{i} M_{\mathrm{Z}} \Gamma_{\mathrm{Z}}}\right. \\
& \left.+\frac{\hat{F}_{q q Z, \text { weak }}^{\sigma}(\hat{s})-\hat{F}_{q q Z, \text { weak }}^{\sigma}\left(M_{\mathrm{Z}}^{2}\right)+\hat{F}_{l l Z, \text { weak }}^{\tau}(\hat{s})-\hat{F}_{l l Z, \text { weak }}^{\tau}\left(M_{\mathrm{Z}}^{2}\right)}{\hat{s}-M_{\mathrm{Z}}^{2}}\right],
\end{aligned}
$$

while the non-resonant contributions involving photon exchange are kept unchanged. Off resonance the introduction of the finite Z-decay width $\Gamma_{Z}$ in the denominator of the vertex corrections changes the amplitude only in $\mathcal{O}\left(\alpha^{2}\right)$ relative to LO, i.e. the effect is beyond NLO.

The treatment of the self-energy corrections is somewhat more involved and requires the inclusion of the LO amplitude. The sum of the LO and self-energy contributions is modified as follows,

$$
\begin{aligned}
f_{q \bar{q}}^{\mathrm{LO}, \sigma \tau}+f_{q \bar{q}, \text { weak }}^{\text {self } \sigma \tau}=- & e^{2}\left\{\frac{Q_{q} Q_{l}}{\hat{s}}\left[1-\frac{\hat{\Sigma}_{\mathrm{T}}^{\gamma \gamma}(\hat{s})}{\hat{s}}\right]+\frac{g_{q q Z}^{\sigma} g_{l l Z}^{\tau}}{\hat{s}-M_{\mathrm{Z}}^{2}}\left[1-\frac{\hat{\Sigma}_{\mathrm{T}}^{\mathrm{ZZ}}(\hat{s})}{\hat{s}-M_{\mathrm{Z}}^{2}}\right]\right. \\
& \left.+\frac{Q_{l} g_{q q Z}^{\sigma}+Q_{q} g_{l l Z}^{\tau}}{\hat{s}} \frac{\hat{\Sigma}_{\mathrm{T}}^{\gamma \mathrm{Z}}(\hat{s})}{\hat{s}-M_{\mathrm{Z}}^{2}}\right\}
\end{aligned}
$$




$$
\begin{aligned}
&=-e^{2}\left\{\frac{Q_{q} Q_{l}}{\hat{s}}\left[1-\frac{\hat{\Sigma}_{\mathrm{T}}^{\gamma \gamma}(\hat{s})}{\hat{s}}\right]+\frac{g_{q q Z}^{\sigma} g_{l l Z}^{\tau}}{\hat{s}-M_{\mathrm{Z}}^{2}}\left[1-\frac{\hat{\Sigma}_{\mathrm{T}}^{\mathrm{ZZ}}\left(M_{\mathrm{Z}}^{2}\right)}{\hat{s}-M_{\mathrm{Z}}^{2}}\right.\right. \\
&\left.-\hat{\Sigma}_{\mathrm{T}}^{\prime \mathrm{ZZ}}\left(M_{\mathrm{Z}}^{2}\right)-\frac{\hat{\Sigma}_{\mathrm{T}}^{\mathrm{ZZ}}(\hat{s})-\hat{\Sigma}_{\mathrm{T}}^{\mathrm{ZZ}}\left(M_{\mathrm{Z}}^{2}\right)-\left(\hat{s}-M_{\mathrm{Z}}^{2}\right) \hat{\Sigma}_{\mathrm{T}}^{\prime \mathrm{ZZ}}\left(M_{\mathrm{Z}}^{2}\right)}{\hat{s}-M_{\mathrm{Z}}^{2}}\right] \\
&+\left.\frac{Q_{l} g_{q q Z}^{\sigma}+Q_{q} g_{l l Z}^{\tau}}{\hat{s}}\left[\frac{\hat{\Sigma}_{\mathrm{T}}^{\gamma \mathrm{Z}}\left(M_{\mathrm{Z}}^{2}\right)}{\hat{s}-M_{\mathrm{Z}}^{2}}+\frac{\hat{\Sigma}_{\mathrm{T}}^{\gamma \mathrm{Z}}(\hat{s})-\hat{\Sigma}_{\mathrm{T}}^{\gamma \mathrm{Z}}\left(M_{\mathrm{Z}}^{2}\right)}{\hat{s}-M_{\mathrm{Z}}^{2}}\right]\right\} \\
& \rightarrow-e^{2}\left\{\frac{Q_{q} Q_{l}}{\hat{s}}\left[1-\frac{\hat{\Sigma}_{\mathrm{T}}^{\gamma \gamma}(\hat{s})}{\hat{s}}\right]+g_{q q Z}^{\sigma} g_{l l Z}^{\tau}\left[\frac{1-\hat{\Sigma}_{\mathrm{T}}^{\prime Z Z}\left(M_{\mathrm{Z}}^{2}\right)}{\hat{s}-M_{\mathrm{Z}}^{2}+\mathrm{i} M_{\mathrm{Z}} \Gamma_{\mathrm{Z}}}\right.\right. \\
&\left.+\frac{\hat{\Sigma}_{\mathrm{T}}^{\mathrm{ZZ}}(\hat{s})-\hat{\Sigma}_{\mathrm{T}}^{\mathrm{ZZ}}\left(M_{\mathrm{Z}}^{2}\right)-\left(\hat{s}-M_{\mathrm{Z}}^{2}\right) \hat{\Sigma}_{\mathrm{T}}^{\prime \mathrm{ZZ}}\left(M_{\mathrm{Z}}^{2}\right)}{\left(\hat{s}-M_{\mathrm{Z}}^{2}\right)^{2}}\right] \\
&+\left(Q_{l} g_{q q Z}^{\sigma}+Q_{q} g_{l l Z}^{\tau}\right)\left[\frac{\hat{\Sigma}_{\mathrm{T}}^{\gamma \mathrm{Z}}\left(M_{\mathrm{Z}}^{2}\right)}{M_{\mathrm{Z}}^{2}-M_{\mathrm{Z}}^{2}+\mathrm{i} M_{\mathrm{Z}} \Gamma_{\mathrm{Z}}}\right. \\
&+\left.\left.\frac{1}{\hat{s}-M_{\mathrm{Z}}^{2}}\left(\frac{\hat{\Sigma}_{\mathrm{T}}^{\gamma \mathrm{Z}}(\hat{s})}{\hat{s}}-\frac{\hat{\Sigma}_{\mathrm{T}}^{\gamma \mathrm{Z}}\left(M_{\mathrm{Z}}^{2}\right)}{M_{\mathrm{Z}}^{2}}\right)\right]\right\}
\end{aligned}
$$

with $\hat{\Sigma}_{\mathrm{T}}^{\prime Z Z}(\hat{s})=\partial \hat{\Sigma}_{\mathrm{T}}^{\mathrm{ZZ}}(\hat{s}) / \partial \hat{s}$. Here we have used the fact that in the on-shell renormalization scheme the renormalized Z-boson self-energy fulfills $\operatorname{Re} \hat{\Sigma}_{\mathrm{T}}^{Z Z}\left(M_{\mathrm{Z}}^{2}\right)=0$ and that the resummed terms account for some imaginary parts via $\operatorname{Im} \hat{\Sigma}_{\mathrm{T}}^{Z Z}\left(M_{\mathrm{Z}}^{2}\right)=M_{\mathrm{Z}} \Gamma_{\mathrm{Z}}$, which holds in $\mathcal{O}(\alpha)$. Off resonance the modification changes the amplitude only in $\mathcal{O}\left(\alpha^{2}\right)$, i.e. beyond NLO. In the resonance region $\left(\hat{s} \approx M_{\mathrm{Z}}^{2}\right)$ the terms involving $\Gamma_{\mathrm{Z}}$ in the denominators do not count as $\mathcal{O}(\alpha)$ corrections, but as LO terms. Thus, in order to achieve NLO accuracy there, $\Gamma_{\mathrm{Z}}$ has to be inserted with NLO precision (or better), or the experimental value should be used. Since the residue of the propagator is a gauge-independent quantity, this modification can be done in the resonant parts without spoiling gauge invariance. In our numerical evaluation we use the experimental value. We finally note that the result (3.39) of the PS substitution can also be obtained upon considering the resonance region of an amplitude that results from the full Dyson summation of the matrix propagator of the $\gamma / \mathrm{Z}$ system (see, e.g., refs. [77, 78]).

The weak box corrections do not become resonant, so that they are not modified in the pole scheme.

(iii) Factorization scheme.

As a third option to define the weak corrections, we make use of the fact that the relative weak corrections $\delta_{q \bar{q} \text {,weak }}^{\text {virt }}$ to the differential partonic cross sections are regular functions of $\hat{s}$, even in the resonance region $\left(\hat{s} \rightarrow M_{\mathrm{Z}}^{2}\right)$ without introducing a finite $\mathrm{Z}$ width. For the virtual photonic corrections this is not the case because of the appearance of corrections proportional to $\alpha \ln \left(\hat{s}-M_{\mathrm{Z}}^{2}\right)$. We, thus, can define the weak NLO correction to the differential partonic cross section in the FS scheme by

$$
\left.\mathrm{d} \hat{\sigma}_{q \bar{q}, \text { weak }}\right|_{\mathrm{FS}}=\left.\delta_{q \bar{q}, \text { weak }}^{\mathrm{virt}}\right|_{\Gamma_{\mathrm{Z}}=0, \delta M_{\mathrm{Z}}=\Sigma_{\mathrm{T}}^{\mathrm{ZZ}}\left(M_{\mathrm{Z}}^{2}\right)} \times \mathrm{d} \hat{\sigma}_{q \bar{q}}^{\mathrm{LO}},
$$



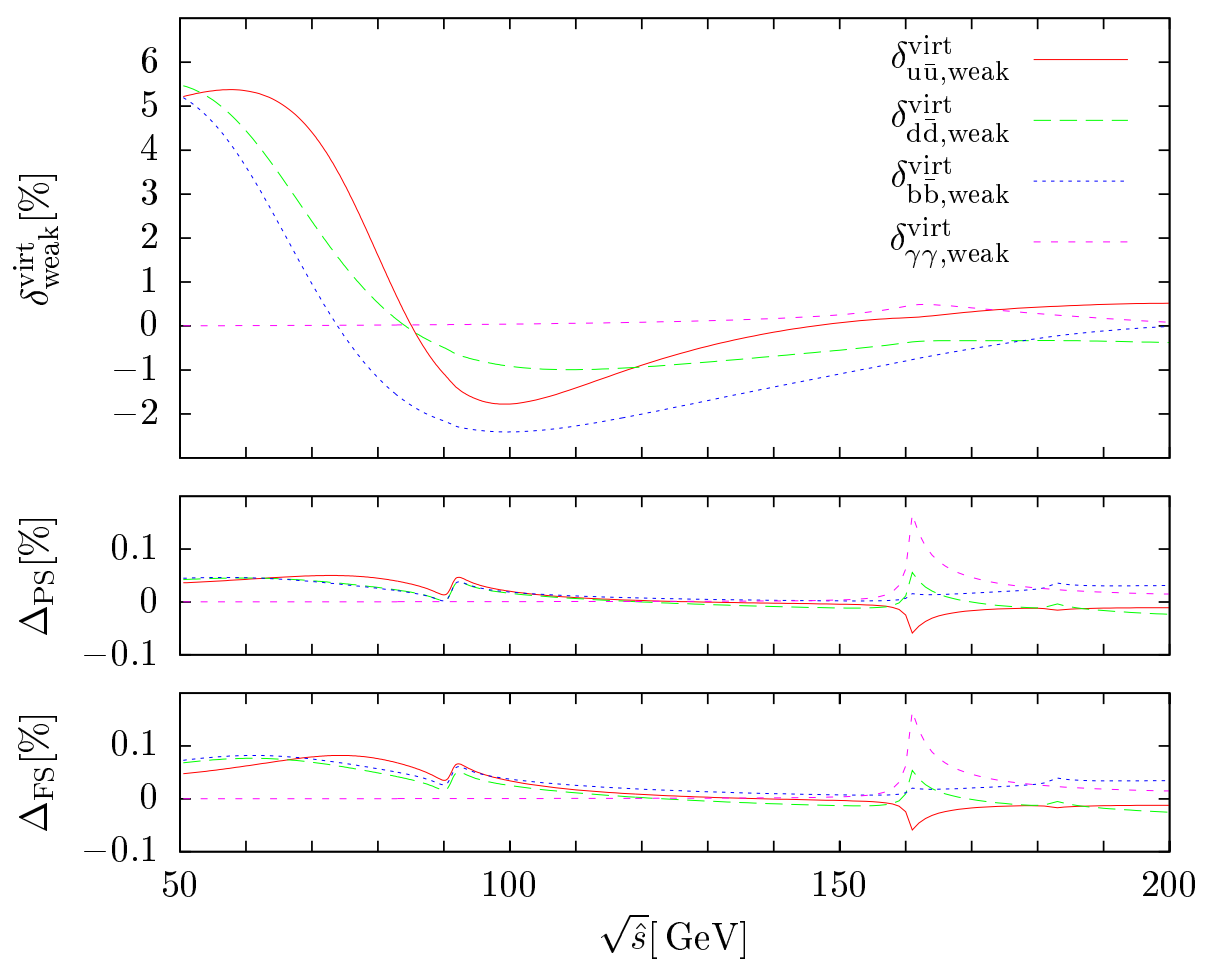

Figure 6. Weak corrections $\delta_{q \bar{q} \text {,weak }}^{\text {virt }}$ and $\delta_{\gamma \gamma \text {,weak }}^{\text {virt }}$ to the total partonic cross sections for the different initial states and the differences $\Delta_{\mathrm{X}}$ between scheme $\mathrm{X}$ and the CMS for treating the $\mathrm{Z}$ resonance.

where the LO cross section $\mathrm{d} \hat{\sigma}_{q \bar{q}}^{\mathrm{LO}}$, as given in (2.12), contains the $\mathrm{Z}$ resonance structure. The subscripts on $\delta_{q \bar{q}, \text { weak }}^{\text {virt }}$ indicate that the $\mathrm{Z}$ width is set to zero everywhere and that the Z-mass counterterm is derived from the full on-shell Z-boson self-energy (i.e. including both real and imaginary parts), in order to avoid double counting of the width effect already present in the LO cross section. This simple scheme respects gauge invariance, because the LO contribution does (see section 2) and the relative correction is derived from the ratio of two gauge-invariant quantities, viz. the weak correction and the LO contribution without any Dyson summation.

As in the PS, the FS calculation only employs real masses and couplings; the width $\Gamma_{Z}$ merely enters the LO cross section. The input-parameter schemes are defined in complete analogy to the PS.

In figure 6 we show the relative weak corrections $\delta_{q \bar{q} \text {,weak }}^{\mathrm{virt}}$ to the total partonic $q \bar{q}$ cross sections for incoming up-type, down-type, and b-quarks, and $\delta_{\gamma \gamma \text {,weak }}^{\text {virt }}$, the weak corrections to $\gamma \gamma \rightarrow l^{-} l^{+}$. The difference between $\delta_{\mathrm{d} d \text {,weak }}^{\text {virt }}$ and $\delta_{\mathrm{b} \overline{\mathrm{b}} \text {,weak }}^{\text {virt }}$ is due to diagrams involving $\mathrm{W}$ bosons and top quarks. It turns out that the differences

$$
\Delta_{\mathrm{X}}=\left.\delta_{q \bar{q}, \text { weak }}^{\mathrm{virt}}\right|_{\mathrm{X}}-\left.\delta_{q \bar{q}, \text { weak }}^{\mathrm{virt}}\right|_{\mathrm{CMS}}
$$

between the different schemes for treating the resonance are below one per mille (apart from the W-pair threshold at $\sqrt{\hat{s}}=2 M_{\mathrm{W}}$ where only the CMS delivers smooth results). 
We, therefore, conclude that all three schemes are equally good in describing the weak corrections to the $\mathrm{Z}$ resonance.

\subsection{Higher-order electroweak effects}

In this section we describe the inclusion of leading higher-order electroweak corrections to the parton processes $q \bar{q} \rightarrow \gamma / \mathrm{Z} \rightarrow l^{-} l^{+}$. First, we discuss the inclusion of the leading universal higher-order corrections originating from the renormalization of the electroweak couplings in the various input-parameter schemes. Second, we consider the leading electroweak corrections in the Sudakov regime which are enhanced by large logarithms $\ln ^{2}\left(\hat{s} / M_{\mathrm{W}}^{2}\right)$. Last we discuss the inclusion of multi-photon final-state radiation, which is treated using the structure-function approach.

\subsubsection{Leading electroweak effects and choice of couplings}

At moderate scales the leading electroweak non-photonic corrections in the SM are due to the running of the electromagnetic coupling $e^{2}=4 \pi \alpha$ from zero-momentum transfer to the electroweak scale, and the large mass splitting between the bottom and the top quark and the associated breaking of the weak isospin symmetry. These leading effects are usually quantified by $\Delta \alpha$ and $\Delta \rho$, respectively, and their two-loop effects can be included in a straightforward way, as described in refs. [66, 97, 101, 102]. Starting from the calculation within the on-shell renormalization scheme with the electromagnetic coupling fixed by $\alpha(0)$, i.e. within the $\alpha(0)$ input parameter scheme defined above, the corrections associated with the running of $\alpha$ are included by the resummation of $\Delta \alpha$ via the substitution

$$
\alpha(0) \rightarrow \alpha\left(M_{\mathrm{Z}}\right)=\frac{\alpha(0)}{1-\Delta \alpha\left(M_{\mathrm{Z}}\right)}
$$

in the LO prediction, where $\Delta \alpha$ is defined in (3.34). For $\Delta \rho$ the leading effects are taken into account via the replacements

$$
s_{\mathrm{W}}^{2} \rightarrow \bar{s}_{\mathrm{W}}^{2} \equiv s_{\mathrm{W}}^{2}+\Delta \rho c_{\mathrm{W}}^{2}, \quad c_{\mathrm{W}}^{2} \rightarrow \bar{c}_{\mathrm{W}}^{2} \equiv 1-\bar{s}_{\mathrm{W}}^{2}=(1-\Delta \rho) c_{\mathrm{W}}^{2} .
$$

This recipe is correct up to $\mathcal{O}\left(\Delta \rho^{2}\right)$ and also reproduces correctly terms of $\mathcal{O}\left(\Delta \alpha\left(M_{\mathrm{Z}}\right) \Delta \rho\right)[101,102]$ in processes with four light external fermions. Note that in $\mathcal{O}\left(\Delta \rho^{2}\right)$ both one- and two-loop corrections to $\Delta \rho$ become relevant; explicitly we use the result

$$
\Delta \rho=3 x_{\mathrm{t}}\left[1+\rho^{(2)}\left(M_{\mathrm{H}}^{2} / m_{\mathrm{t}}^{2}\right) x_{\mathrm{t}}\right]\left[1-\frac{2 \alpha_{\mathrm{s}}}{9 \pi}\left(\pi^{2}+3\right)\right], \quad 3 x_{\mathrm{t}}=\frac{3 \sqrt{2} G_{\mu} m_{\mathrm{t}}^{2}}{16 \pi^{2}}=\left.\Delta \rho^{(1)}\right|^{G_{\mu}},
$$

with the function $\rho^{(2)}$ given in eq. (12) of ref. [103]. In the following we isolate the genuine two-loop effects induced by $\Delta \alpha$ and $\Delta \rho$ after properly subtracting the corresponding oneloop contributions $\Delta \alpha$ and $\Delta \rho^{(1)}$ already contained in the full NLO electroweak corrections.

The leading one- and two-loop effects of $\Delta \alpha$ and $\Delta \rho$ in the $\alpha(0)$ scheme are included in the LO cross section (2.12) upon performing the substitutions (3.42) and (3.43). In this context, the basic ingredients in $(2.12)$ are the products $\alpha(0)^{2} Q_{q}^{2} Q_{l}^{2}, \alpha(0)^{2} Q_{q} Q_{l} g_{q q Z}^{\sigma} g_{l l Z}^{\tau}$, 
and $\alpha(0)^{2}\left(g_{q q Z}^{\sigma} g_{l l Z}^{\tau}\right)^{2}$ of the electroweak couplings defined in (2.7). In the following we define $\bar{g}_{f f Z}^{\sigma}$ to result from $g_{f f Z}^{\sigma}$ upon applying (3.43). Carrying out the above substitutions and keeping terms up to two-loop order, the results for these products can be written as

$$
\begin{aligned}
\alpha(0)^{2} Q_{q}^{2} Q_{l}^{2} \rightarrow & \alpha\left(M_{\mathrm{Z}}\right)^{2} Q_{q}^{2} Q_{l}^{2}=\alpha(0)^{2} Q_{q}^{2} Q_{l}^{2}\left[1+2 \Delta \alpha\left(M_{\mathrm{Z}}\right)+3 \Delta \alpha\left(M_{\mathrm{Z}}\right)^{2}+\ldots\right], \\
\alpha(0)^{2} Q_{q} Q_{l} g_{q q Z}^{\sigma} g_{l l Z}^{\tau} \rightarrow & \alpha\left(M_{\mathrm{Z}}\right)^{2} Q_{q} Q_{l} \bar{g}_{q q Z}^{\sigma} \bar{g}_{l l Z}^{\tau} \\
= & \alpha(0)^{2} Q_{q} Q_{l}\left\{g_{q q Z}^{\sigma} g_{l l Z}^{\tau}\left[1+2 \Delta \alpha\left(M_{\mathrm{Z}}\right)+3 \Delta \alpha\left(M_{\mathrm{Z}}\right)^{2}\right]\right. \\
& \left.+\Delta \rho a_{q l}^{\sigma \tau}+\Delta \rho^{2} b_{q l}^{\sigma \tau}+2 \Delta \alpha\left(M_{\mathrm{Z}}\right) \Delta \rho a_{q l}^{\sigma \tau}+\ldots\right\}, \\
\alpha(0)^{2}\left(g_{q q Z}^{\sigma} g_{l l Z}^{\tau}\right)^{2} \rightarrow \alpha\left(M_{\mathrm{Z}}\right)^{2}\left(\bar{g}_{q q Z}^{\sigma} \bar{g}_{l l Z}^{\tau}\right)^{2} & \alpha(0)^{2}\left\{\left(g_{q q Z}^{\sigma} g_{l l Z}^{\tau}\right)^{2}\left[1+2 \Delta \alpha\left(M_{\mathrm{Z}}\right)+3 \Delta \alpha\left(M_{\mathrm{Z}}\right)^{2}\right]\right. \\
& \quad+2 \Delta \rho a_{q l}^{\sigma \tau} g_{q q Z}^{\sigma} g_{l l Z}^{\tau}+\Delta \rho^{2}\left(\left(a_{q l}^{\sigma \tau}\right)^{2}+2 b_{q l}^{\sigma \tau} g_{q q Z}^{\sigma} g_{l l Z}^{\tau}\right) \\
& \left.+4 \Delta \alpha\left(M_{\mathrm{Z}}\right) \Delta \rho a_{q l}^{\sigma \tau} g_{q q Z}^{\sigma} g_{l l Z}^{\tau}+\ldots\right\}
\end{aligned}
$$

where we have introduced the shorthands

$$
a_{q l}^{\sigma \tau}=\frac{Y_{q^{\sigma}} Y_{l^{\tau}}}{4 c_{\mathrm{w}}^{2}}-\frac{c_{\mathrm{W}}^{2} I_{\mathrm{w}, q^{\sigma}}^{3} I_{\mathrm{w}, l^{\tau}}^{3}}{s_{\mathrm{W}}^{4}}, \quad b_{q l}^{\sigma \tau}=\frac{Y_{q^{\sigma}} Y_{l^{\tau}}}{4 c_{\mathrm{w}}^{2}}+\frac{c_{\mathrm{W}}^{4} I_{\mathrm{w}, q^{\sigma}}^{3} I_{\mathrm{w}, l^{\tau}}^{3}}{s_{\mathrm{W}}^{6}},
$$

with $Y_{f^{\sigma}}=2\left(Q_{f}-I_{\mathrm{w}, f^{\sigma}}^{3}\right)$ denoting the weak hypercharge of fermion $f^{\sigma}$ with chirality $\sigma= \pm$. Dropping the LO contribution and subtracting the relevant one-loop terms, which are proportional to $\Delta \alpha\left(M_{\mathrm{Z}}\right)$ and $\Delta \rho^{(1)}$, the leading two-loop contributions to the coupling combinations read

$$
\begin{aligned}
\left.\alpha^{2} Q_{q}^{2} Q_{l}^{2}\right|_{\mathrm{LL}^{2}} ^{\alpha(0)}= & 3 \alpha(0)^{2} Q_{q}^{2} Q_{l}^{2} \Delta \alpha\left(M_{\mathrm{Z}}\right)^{2} \\
\left.\alpha^{2} Q_{q} Q_{l} g_{q q Z}^{\sigma} g_{l l Z}^{\tau}\right|_{\mathrm{LL}^{2}} ^{\alpha(0)}=\alpha(0)^{2} Q_{q} Q_{l} & \left\{3 g_{q q Z}^{\sigma} g_{l l Z}^{\tau} \Delta \alpha\left(M_{\mathrm{Z}}\right)^{2}+\left(\Delta \rho-\left.\Delta \rho^{(1)}\right|^{\alpha(0)}\right) a_{q l}^{\sigma \tau}\right. \\
& \left.+\Delta \rho^{2} b_{q l}^{\sigma \tau}+2 \Delta \alpha\left(M_{\mathrm{Z}}\right) \Delta \rho a_{q l}^{\sigma \tau}\right\} \\
\left.\alpha^{2}\left(g_{q q Z}^{\sigma} g_{l l Z}^{\tau}\right)^{2}\right|_{\mathrm{LL}^{2}} ^{\alpha(0)}=\alpha(0)^{2}\{ & 3\left(g_{q q Z}^{\sigma} g_{l l Z}^{\tau}\right)^{2} \Delta \alpha\left(M_{\mathrm{Z}}\right)^{2} \\
& +2\left(\Delta \rho-\left.\Delta \rho^{(1)}\right|^{\alpha(0)}\right) a_{q l}^{\sigma \tau} g_{q q Z}^{\sigma} g_{l l Z}^{\tau} \\
& +\Delta \rho^{2}\left(\left(a_{q l}^{\sigma \tau}\right)^{2}+2 b_{q l}^{\sigma \tau} g_{q q Z}^{\sigma} g_{l l Z}^{\tau}\right) \\
& \left.+4 \Delta \alpha\left(M_{\mathrm{Z}}\right) \Delta \rho a_{q l}^{\sigma \tau} g_{q q Z}^{\sigma} g_{l l Z}^{\tau}\right\}
\end{aligned}
$$

where we have indicated the $\alpha(0)$ input parameter scheme by superscripts. In $\Delta \rho^{(1)}$ the superscript means which value of $\alpha$ is used in its evaluation. 
The transition from the $\alpha(0)$ to the $\alpha\left(M_{\mathrm{Z}}\right)$ scheme is rather easy. Since $\alpha\left(M_{\mathrm{Z}}\right)$ is already used as LO coupling, only the replacement (3.43) applies, but not (3.42). Thus, starting from the formulas in the $\alpha(0)$ scheme given above, the terms involving $\Delta \alpha\left(M_{\mathrm{Z}}\right)$ should be dropped, and we obtain for the leading two-loop terms

$$
\begin{aligned}
\left.\alpha^{2} Q_{q}^{2} Q_{l}^{2}\right|_{\mathrm{LL}^{2}} ^{\alpha\left(M_{\mathrm{Z}}\right)}= & 0, \\
\left.\alpha^{2} Q_{q} Q_{l} g_{q q Z}^{\sigma} g_{l l Z}^{\tau}\right|_{\mathrm{LL}^{2}} ^{\alpha\left(M_{\mathrm{Z}}\right)}= & \alpha\left(M_{\mathrm{Z}}\right)^{2} Q_{q} Q_{l}\left\{\left(\Delta \rho-\left.\Delta \rho^{(1)}\right|^{\alpha\left(M_{\mathrm{Z}}\right)}\right) a_{q l}^{\sigma \tau}+\Delta \rho^{2} b_{q l}^{\sigma \tau}\right\}, \\
\left.\alpha^{2}\left(g_{q q Z}^{\sigma} g_{l l Z}^{\tau}\right)^{2}\right|_{\mathrm{LL}^{2}} ^{\alpha\left(M_{\mathrm{Z}}\right)}= & \alpha\left(M_{\mathrm{Z}}\right)^{2}\left\{2\left(\Delta \rho-\left.\Delta \rho^{(1)}\right|^{\alpha\left(M_{\mathrm{Z}}\right)}\right) a_{q l}^{\sigma \tau} g_{q q Z}^{\sigma} g_{l l Z}^{\tau}\right. \\
& \left.+\Delta \rho^{2}\left(\left(a_{q l}^{\sigma \tau}\right)^{2}+2 b_{q l}^{\sigma \tau} g_{q q Z}^{\sigma} g_{l l Z}^{\tau}\right)\right\},
\end{aligned}
$$

In the $G_{\mu}$-scheme, $\alpha_{G_{\mu}}$ effectively involves a factor $\alpha\left(M_{\mathrm{Z}}\right) s_{\mathrm{W}}^{2}$, so that the basic replacements read $\alpha_{G_{\mu}} \rightarrow \alpha_{G_{\mu}} \bar{s}_{\mathrm{W}}^{2} / s_{\mathrm{W}}^{2}$ and $g_{f f Z}^{\sigma} \rightarrow \bar{g}_{f f Z}^{\sigma}$. This procedure leads to the following leading two-loop terms,

$$
\begin{aligned}
\left.\alpha^{2} Q_{q}^{2} Q_{l}^{2}\right|_{\mathrm{LL}^{2}} ^{G_{\mu}}= & \alpha_{G_{\mu}}^{2} Q_{q}^{2} Q_{l}^{2}\{ \\
\left.\alpha^{2} Q_{q} Q_{l} g_{q q Z}^{\sigma} g_{l l Z}^{\tau}\right|_{\mathrm{LL}^{2}} ^{G_{\mu}}=\alpha_{G_{\mu}}^{2} Q_{q} Q_{l}\{ & \left.\left(\Delta \rho-\left.\Delta \rho^{(1)}\right|^{G_{\mu}}\right) \frac{c_{\mathrm{W}}^{2}}{s_{\mathrm{W}}^{2}}+\Delta \rho^{2} \frac{c_{\mathrm{W}}^{4}}{s_{\mathrm{W}}^{4}}\right\},\left(a_{q l}^{\sigma \tau}+\frac{2 c_{\mathrm{W}}^{2}}{s_{\mathrm{W}}^{2}} g_{q q Z}^{\sigma} g_{l l Z}^{\tau}\right) \\
& \left.+\Delta \rho^{2}\left(b_{q l}^{\sigma \tau}+\frac{2 c_{\mathrm{W}}^{2}}{s_{\mathrm{W}}^{2}} a_{q l}^{\sigma \tau}+\frac{c_{\mathrm{W}}^{4}}{s_{\mathrm{W}}^{4}} g_{q q Z}^{\sigma} g_{l l Z}^{\tau}\right)\right\}, \\
\left.\alpha^{2}\left(g_{q q Z}^{\sigma} g_{l l Z}^{\tau}\right)^{2}\right|_{\mathrm{LL}^{2}} ^{G_{\mu}}=\alpha_{G_{\mu}}^{2}\left\{2\left(\Delta \rho-\left.\Delta \rho^{(1)}\right|^{G_{\mu}}\right) g_{q q Z}^{\sigma} g_{l l Z}^{\tau}\left(a_{q l}^{\sigma \tau}+\frac{c_{\mathrm{W}}^{2}}{s_{\mathrm{W}}^{2}} g_{q q Z}^{\sigma} g_{l l Z}^{\tau}\right)\right. & +\Delta \rho^{2}\left[\left(a_{q l}^{\sigma \tau}\right)^{2}+2 g_{q q Z}^{\sigma} g_{l l Z}^{\tau}\left(b_{q l}^{\sigma \tau}+\frac{2 c_{\mathrm{W}}^{2}}{s_{\mathrm{W}}^{2}} a_{q l}^{\sigma \tau}\right)\right. \\
& \left.\left.+\frac{c_{\mathrm{W}}^{4}}{s_{\mathrm{W}}^{4}}\left(g_{q q Z}^{\sigma} g_{l l Z}^{\tau}\right)^{2}\right]\right\} .
\end{aligned}
$$

We recall that in the CC case [42] the $G_{\mu}$ scheme absorbs the full $\Delta \alpha$ and $\Delta \rho$ terms into the LO prediction (at least up to two loops), because the CC coupling factor $\alpha_{G_{\mu}} / s_{\mathrm{W}}^{2}$ does not receive such universal corrections. In the present $\mathrm{NC}$ case this absorption is not complete, and only a numerical analysis can assess the size of the remaining explicit universal two-loop corrections.

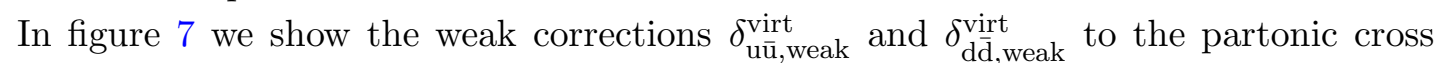
sections for the different input parameter schemes, including the corresponding higher-order corrections due to $\Delta \alpha$ and $\Delta \rho$. It is clearly visible that the impact of the universal twoloop corrections is largest in the $\alpha(0)$ scheme and smallest in the $G_{\mu}$ scheme, as expected. We, therefore, conclude that the $G_{\mu}$ scheme should be the most stable w.r.t. higher-order electroweak effects among the discussed input parameter schemes. From the above formulas it is also clear that none of the schemes is fully optimized to absorb the effects of $\Delta \alpha$ and 

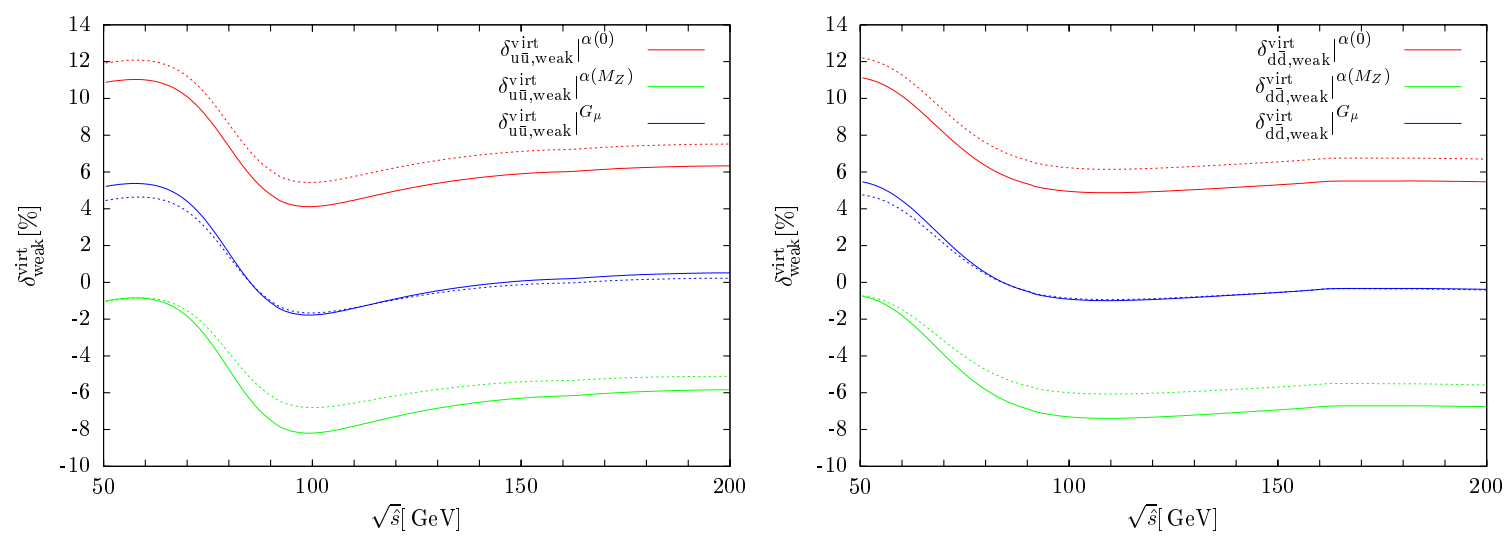

Figure 7. Weak corrections $\delta_{\mathrm{u} u \text {,weak }}^{\mathrm{virt}}$ and $\delta_{\mathrm{d} \overline{\mathrm{d}} \text {,weak }}^{\mathrm{virt}}$ to the partonic cross sections for the different input parameter schemes, with (dashed lines) and without leading higher-order corrections due to $\Delta \alpha$ and $\Delta \rho$.

$\Delta \rho$ into the LO prediction as much as possible. While the $\alpha\left(M_{\mathrm{Z}}\right)$ scheme is more suited for photon exchange, where no leading $\Delta \rho$ corrections arise, the $G_{\mu}$ scheme describes Zexchange diagrams better, because the generic $\mathrm{NC}$ coupling $e /\left(s_{\mathrm{W}} c_{\mathrm{W}}\right)$ is closer to the weak gauge coupling $e / s_{\mathrm{W}}$ than to $e$. In view of the accuracy required for hadron collider physics we do not see, however, the necessity to switch to a mixed optimized scheme and take the $G_{\mu}$ scheme as default in the following.

\subsubsection{Leading weak corrections in the Sudakov regime}

For dilepton production at large lepton transverse momenta, the parton kinematics is restricted to the Sudakov regime, characterized by large Mandelstam parameters $\hat{s}$, $|\hat{t}|$, $|\hat{u}| \gg M_{\mathrm{W}}^{2}$. The structure of electroweak corrections beyond $\mathcal{O}(\alpha)$ in this high-energy regime has been investigated in detail by several groups in recent years (see e.g. refs. [104$112]$ and references therein).

As described for example in refs. [109, 112], the leading electroweak logarithmic corrections, which are enhanced by large factors $L=\ln \left(\hat{s} / M_{\mathrm{W}}^{2}\right)$, can be divided into an $\mathrm{SU}(2) \times \mathrm{U}(1)$-symmetric part, an electromagnetic part, and a subleading part induced by the mass difference between $\mathrm{W}$ and $\mathrm{Z}$ bosons. The last part does not contribute to corrections $\propto\left(\alpha L^{2}\right)^{n}$ and is neglected in the following. The leading (Sudakov) logarithms $\propto\left(\alpha L^{2}\right)^{n}$ of electromagnetic origin cancel between virtual and real (soft) bremsstrahlung corrections; for the subleading logarithms such cancellations should strongly depend on the observable under consideration. The only source of leading logarithms is, thus, the symmetric electroweak (sew) part, which can be characterized by comprising $\mathrm{W}$ bosons, $\mathrm{Z}$ bosons, and photons of a common mass $M_{\mathrm{W}}$. In the following we consider this type of corrections to the $q \bar{q}$ annihilation channels of the light quarks, i.e. $q=\mathrm{u}, \mathrm{d}, \mathrm{c}, \mathrm{s}$, which deliver the dominating contribution to the dilepton cross section.

The one-loop correction $\delta_{q \bar{q}, \text { sew }}^{(1), \sigma \tau}$ to the squared amplitude, with chiralities $\sigma$ and $\tau$ as defined above, can be obtained by expanding the full result for the virtual correction $\delta_{q \bar{q}}^{\text {virt }, \sigma \tau}$ 
(given in the appendix) for large $\hat{s},|\hat{t}|,|\hat{u}| \gg M_{\mathrm{W}}^{2}$. The explicit result can be written as

$$
\delta_{q \bar{q}, \text { sew }}^{(1), \sigma \tau}=\frac{\alpha}{2 \pi}\left\{-L^{2} C_{1, \mathrm{NC}}^{\mathrm{sew}, \sigma \tau}+L C_{1, \mathrm{NC}}^{\mathrm{ad}, \sigma \tau} / C_{0, \mathrm{NC}}^{\sigma \tau}\right\}
$$

with factors

$$
\begin{aligned}
C_{0, \mathrm{NC}}^{\sigma \tau} & =e^{2}\left(g_{q q Z}^{\sigma} g_{l l Z}^{\tau}+Q_{q} Q_{l}\right), \\
C_{1, \mathrm{NC}}^{\mathrm{sew}, \sigma \tau} & =\left(g_{q q Z}^{\sigma}\right)^{2}+Q_{q}^{2}+\left(g_{l l Z}^{\tau}\right)^{2}+Q_{l}^{2}+\frac{\delta_{\sigma-}+\delta_{\tau-}}{2 s_{\mathrm{W}}^{2}}, \\
C_{1, \mathrm{NC}}^{\mathrm{ad}, \sigma \tau} & =\frac{4}{e^{2}}\left(C_{0, \mathrm{NC}}^{\sigma \tau}\right)^{2} \ln \left(\frac{\hat{u}}{\hat{t}}\right)+\frac{e^{2}}{s_{\mathrm{W}}^{4}} \delta_{\sigma-} \delta_{\tau-} \ln \left(\frac{-\hat{r}}{\hat{s}}\right) \\
\text { with } \hat{r} & =\left\{\begin{array}{l}
\hat{t} \text { for } I_{\mathrm{W}, q}^{3} I_{\mathrm{W}, l}^{3}>0, \\
\hat{u} \text { for } I_{\mathrm{W}, q}^{3} I_{\mathrm{w}, l}^{3}<0,
\end{array}\right.
\end{aligned}
$$

which have been introduced in section 8.4 .1 of ref. [112]. In eq. (3.50) we did not only include the leading Sudakov logarithms $\propto \alpha L^{2}$, but also the related "angular-dependent" contributions $\alpha \alpha L \ln (-\hat{t} / \hat{s})$ or $\alpha L \ln (-\hat{u} / \hat{s})$. Our explicit $\mathcal{O}(\alpha)$ result is in agreement with the general results presented in refs. $[109,112]$, where the corresponding corrections are also given at the two-loop level. These $\mathcal{O}\left(\alpha^{2}\right)$ corrections can be obtained from the $\mathcal{O}(\alpha)$ result by an appropriate exponentiation [107]. For the leading "sew" corrections (including $\alpha^{2} L^{4}, \alpha^{2} L^{3} \ln (-\hat{t} / \hat{s})$, and $\alpha^{2} L^{3} \ln (-\hat{u} / \hat{s})$ terms $)$ this exponentiation simply reads [112]

$$
\left|\mathcal{M}_{q \bar{q}}\right|^{2} \sim\left|\mathcal{M}_{q \bar{q}}^{\mathrm{LO}}\right|^{2} \exp \left\{\delta_{q \bar{q}, \text { sew }}^{(1)}\right\}=\left|\mathcal{M}_{q \bar{q}}^{\mathrm{LO}}\right|^{2}\left(1+\delta_{q \bar{q}, \text { sew }}^{(1)}+\delta_{q \bar{q}, \text { sew }}^{(2)}+\ldots\right)
$$

with

$$
\delta_{q \bar{q}, \text { sew }}^{(2)}=\left(\frac{\alpha}{2 \pi}\right)^{2}\left\{\frac{1}{2} L^{4}\left(C_{1, \mathrm{NC}}^{\mathrm{sew}}\right)^{2}-L^{3} C_{1, \mathrm{NC}}^{\mathrm{sew}} C_{1, \mathrm{NC}}^{\mathrm{ad}} / C_{0, \mathrm{NC}}\right\}
$$

where we have suppressed the chirality indices $\sigma, \tau$ in the notation.

Particularly in the case of NC fermion-antifermion scattering processes it was observed $[110,111]$ that large cancellations take place between leading and subleading logarithms. In view of this uncertainty, we do not include the two-loop high-energy logarithms in our full predictions. Instead, we evaluate the leading two-loop part $\delta_{q \bar{q}, \text { sew }}^{(2)}$ as a measure of missing electroweak corrections beyond $\mathcal{O}(\alpha)$ in the high-energy Sudakov regime.

Moreover, since the electroweak high-energy logarithmic corrections are associated with virtual soft and/or collinear weak-boson or photon exchange, they all have counterparts in real weak-boson or photon emission processes which can partially cancel (but not completely, see ref. [105]) the large negative corrections. To which extent the cancellation occurs depends on the experimental possibilities to separate final states with or without weak bosons or photons. This issue is discussed for example in refs. [113, 114]. The numerical analysis presented in ref. [114] demonstrates the effect of real weak-boson emission in the distributions in the transverse lepton momentum $p_{\mathrm{T}, l}$ and in the invariant mass $M_{l l}$ of the lepton pair. At the LHC, at $M_{l l}=2 \mathrm{TeV}$ the electroweak corrections are reduced from about $-11 \%$ to $-8 \%$ by weak-boson emission. At $p_{\mathrm{T}, l}=1 \mathrm{TeV}$ the corresponding reduction from about $-10 \%$ to $-3 \%$ is somewhat larger. This illustrates the sensitivity of weak-boson emission effects to the details of experimental event selection, in particular, how dilepton production is separated from diboson production. 


\subsubsection{Multi-photon final-state radiation}

The emission of photons collinear to the outgoing charged lepton leads to corrections that are enhanced by large logarithms of the form $\alpha \ln \left(m_{l}^{2} / Q^{2}\right)$ with $Q$ denoting a characteristic scale of the process. The Kinoshita-Lee-Nauenberg (KLN) theorem [115, 116] guarantees that these logarithms cancel if photons collinear to the lepton are treated fully inclusively. However, since we apply a phase-space cut on the momentum of the outgoing lepton, contributions enhanced by these logarithms survive if the momentum of the bare lepton is considered, i.e. if no photon recombination is performed. While the concept of a bare lepton is not realistic for electrons, it is phenomenologically relevant for muon final states.

The first-order logarithm $\alpha \ln \left(m_{l}^{2} / Q^{2}\right)$ is, of course, contained in the full $\mathcal{O}(\alpha)$ correction, so that $Q$ is unambiguously fixed in this order. However, it is desirable to control the logarithmically enhanced corrections beyond $\mathcal{O}(\alpha)$. This can be done in the so-called structure-function approach [57-62], where these logarithms are derived from the universal factorization of the related mass singularity. The incorporation of the mass-singular logarithms takes the form of a convolution integral over the LO cross section $\sigma^{\mathrm{LO}}$,

$$
\begin{aligned}
\sigma_{\text {LLFSR }}= & \int \mathrm{d} \sigma^{\mathrm{LO}}\left(p_{1}, p_{2} ; k_{1}, k_{2}\right) \int_{0}^{1} \mathrm{~d} z_{1} \Gamma_{l l}^{\mathrm{LL}}\left(z_{1}, Q^{2}\right) \Theta_{\text {cut }}\left(z_{1} k_{1}\right) \\
& \times \int_{0}^{1} \mathrm{~d} z_{2} \Gamma_{l l}^{\mathrm{LL}}\left(z_{2}, Q^{2}\right) \Theta_{\text {cut }}\left(z_{2} k_{2}\right)
\end{aligned}
$$

where the step function $\Theta_{\text {cut }}$ is equal to 1 if the event passes the cut on the rescaled lepton momentum $z_{i} k_{i}$ and 0 otherwise. The variables $z_{i}$ are the momentum fractions describing the respective lepton energy loss by collinear photon emission. Note that in contrast to the parton-shower approaches to photon radiation (see e.g. refs. $[43,46]$ ), the structure-function approach neglects the photon momenta transverse to the lepton momentum.

For the structure function $\Gamma_{l l}^{\mathrm{LL}}\left(z, Q^{2}\right)$ we take into account terms up to $\mathcal{O}\left(\alpha^{3}\right)$ improved by the well-known exponentiation of the soft-photonic parts [57-62]; our precise formula can also be found in eq. (2.21) of ref. [42]. Technically, we add the cross section (3.54) to the one-loop result and subtract the LO and one-loop contributions

$$
\begin{aligned}
\sigma_{\mathrm{LL}^{1} \mathrm{FSR}}=\int & \mathrm{d} \sigma^{\mathrm{LO}}\left(p_{1}, p_{2} ; k_{1}, k_{2}\right) \int_{0}^{1} \mathrm{~d} z_{1} \int_{0}^{1} \mathrm{~d} z_{2}\left[\delta\left(1-z_{1}\right) \delta\left(1-z_{2}\right)\right. \\
& \left.+\Gamma_{l l}^{\mathrm{LL}, 1}\left(z_{1}, Q^{2}\right) \delta\left(1-z_{2}\right)+\delta\left(1-z_{1}\right) \Gamma_{l l}^{\mathrm{LL}, 1}\left(z_{2}, Q^{2}\right)\right] \Theta_{\text {cut }}\left(z_{1} k_{1}\right) \Theta_{\text {cut }}\left(z_{2} k_{2}\right)
\end{aligned}
$$

contained in (3.54) in order to avoid double counting. The one-loop contribution to the structure function reads

$$
\Gamma_{l l}^{\mathrm{LL}, 1}\left(z, Q^{2}\right)=\frac{\beta_{l}}{4}\left(\frac{1+z^{2}}{1-z}\right)_{+}
$$

with the variable

$$
\beta_{l}=\frac{2 \alpha(0)}{\pi}\left[\ln \left(\frac{Q^{2}}{m_{l}^{2}}\right)-1\right],
$$


which quantifies the large logarithm. In this context it should be noted that both the full photonic one-loop corrections (see section 3.2) and the multi-photon effects discussed in this section are evaluated with $\alpha(0)$ as the photonic coupling in the corrections. Thus, when subtracting the one-loop part of eq. (3.55) from the full one-loop result, the logarithmic terms $\propto \alpha(0) \ln m_{l}$ cancel exactly in all our considered input parameter schemes.

The uncertainty that is connected with the choice of $Q^{2}$ enters in $\mathcal{O}\left(\alpha^{2}\right)$, since all $\mathcal{O}(\alpha)$ corrections, including constant terms, are taken into account. As default we choose the value

$$
Q=\xi \sqrt{\hat{s}}
$$

with $\xi=1$. In order to quantify the scale uncertainty, we vary $\xi$ between $1 / 3$ and 3 .

\section{Radiative corrections to the partonic cross sections in the MSSM}

In this section we examine the effect of corrections to $\mathrm{pp} / \mathrm{p} \overline{\mathrm{p}} \rightarrow \gamma / \mathrm{Z} \rightarrow l^{-} l^{+}+X$ within the supersymmetric extension of the SM. A similar study for the case of W-boson production was performed in ref. [42]. Even though Drell-Yan processes do not represent discovery channels for supersymmetry it is important to study the influence of SUSY on Drell-Yan processes since they will be used at the LHC to calibrate detectors, to monitor luminosity, and to extract information on PDF. Measurements on Drell-Yan processes will also allow for precision tests of the SM and its extensions through radiative corrections. If there were large corrections due to SUSY particles all this information would be biased and therefore not very useful to extract information about the underlying physics.

As an estimate of the impact of supersymmetric extensions of the SM we calculate the SUSY corrections to $\mathrm{pp} / \mathrm{p} \overline{\mathrm{p}} \rightarrow \gamma / \mathrm{Z} \rightarrow l^{-} l^{+}+X$ within the MSSM. As in ref. [42] we calculate the full MSSM corrections and subtract the SM corrections, so that the MSSM corrections can be added to the SM corrections without double counting,

$$
\delta_{q \bar{q}, \mathrm{SUSY}} \equiv \delta_{q \bar{q}, \mathrm{MSSM}}-\delta_{q \bar{q}, \mathrm{SM}}\left(M_{\mathrm{H}}=M_{\mathrm{h}^{0}}\right) .
$$

Note that we identify the mass $M_{\mathrm{H}}$ of the SM Higgs boson with the mass $M_{\mathrm{h}^{0}}$ of the lightest Higgs boson $\mathrm{h}^{0}$ of the MSSM for the subtraction of the SM corrections.

We divide the SUSY corrections into the SUSY-QCD and the SUSY electroweak (SUSY-EW) corrections. The SUSY-QCD corrections are due to corrections to the $q \bar{q} \gamma / Z$ vertices as shown in figure 8 and the quark wave-function renormalization via squark-gluino loops.

To obtain the SUSY-EW corrections, we proceed as in ref. [42] and calculate the complete electroweak $\mathcal{O}(\alpha)$ corrections in the MSSM and subtract the SM corrections. The SUSY-EW corrections can be further divided into Two-Higgs-Doublet-Model (THDM) and pure SUSY corrections. The THDM corrections are due to the extension of the Higgs sector to two Higgs doublets. It is this part of the corrections where we subtract the SM corrections, since in the decoupling limit where the mass $M_{\mathrm{A}^{0}}$ of the pseudoscalar Higgs boson $\mathrm{A}^{0}$ becomes large, both the THDM sector of the MSSM and the SM Higgs sector coincide if we identify the light neutral Higgs boson of the MSSM with the SM Higgs boson. The pure SUSY corrections consist of sfermion, neutralino, and chargino loops (see figures 9 and 10). 


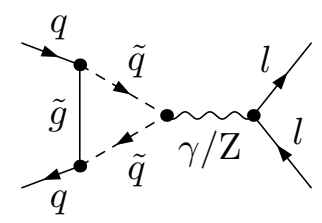

Figure 8. Example diagram for SUSY-QCD corrections, which are due to squark $(\tilde{q})$ and gluino $(\tilde{g})$ exchange.
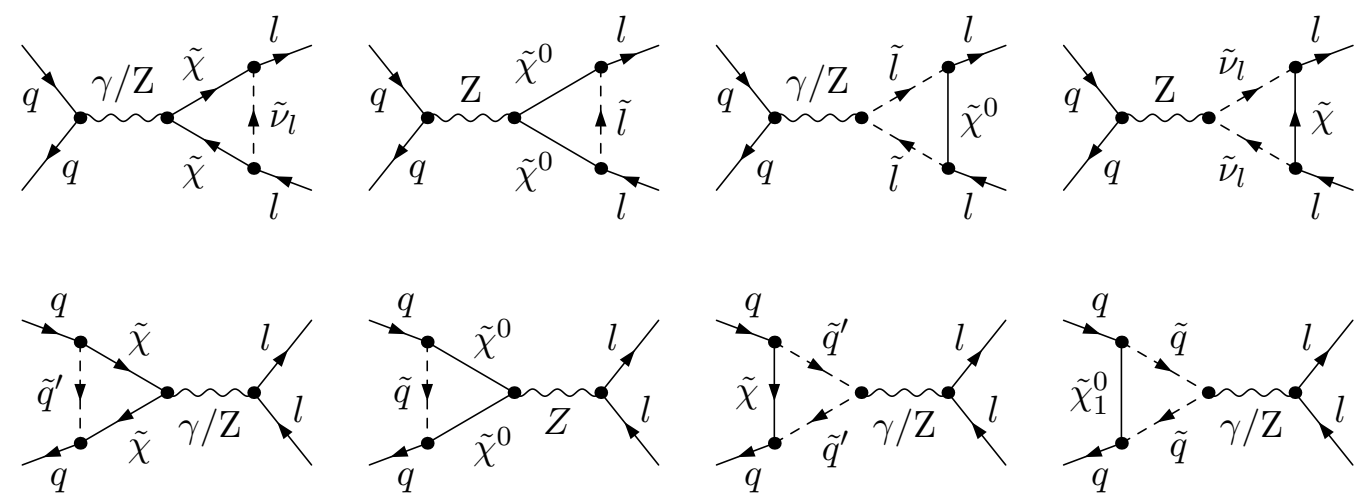

Figure 9. Example diagrams for pure SUSY vertex corrections, which involve squark $(\tilde{q})$, slepton $(\tilde{l})$, sneutrino $\left(\tilde{\nu}_{l}\right)$, chargino $(\tilde{\chi})$, and neutralino $\left(\tilde{\chi}^{0}\right)$ exchange.
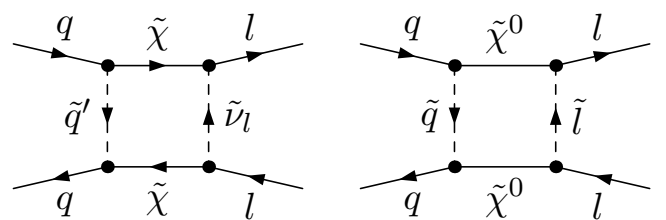

Figure 10. Example diagrams for pure SUSY box corrections, which involve squark $(\tilde{q})$, slepton $(\tilde{l})$, sneutrino $\left(\tilde{\nu}_{l}\right)$, chargino $(\tilde{\chi})$, and neutralino $\left(\tilde{\chi}^{0}\right)$ exchange.

For the computation of the SUSY corrections we have again performed two independent calculations, one using the FEYnARTs/FormCALC/LOOPTOOLS [81-83] framework and the other one using FeynARTs and inhouse Mathematica routines. The calculation is done using the on-shell-scheme as defined in ref. [66]. Since the LO process is a pure SM process the renormalization of ref. [66] can be applied without modification. To treat the resonance at the Z-boson peak we use the LO cross section evaluated in the FS and multiply 
with a correction factor,

$$
\hat{\sigma}_{q \bar{q}, \mathrm{SUSY}}=\left.\delta_{q \bar{q}, \mathrm{SUSY}}\right|_{\Gamma_{\mathrm{Z}}=0} \times\left.\hat{\sigma}_{q \bar{q}}^{\mathrm{LO}}\right|_{\mathrm{FS}},
$$

where the relative SUSY correction $\delta_{q \bar{q}, \mathrm{SUSY}}$, as defined in (4.1), can be evaluated without any special treatment of the Z-boson resonance, i.e. with a zero Z-boson decay width. We find that for the SPS benchmark scenarios [119] (see appendix C) both the SUSY-QCD and the SUSY-EW corrections stay below $2 \%$ for partonic centre-of-mass energies up to $2 \mathrm{TeV}$. As an example we show in figure 11 the partonic LO cross section and radiative corrections for $\mathrm{d} \overline{\mathrm{d}}$ initial-states for the different MSSM scenarios.

\section{$5 \quad$ Numerical results for the cross section $\mathrm{pp} / \mathrm{p} \overline{\mathrm{p}} \rightarrow \gamma / \mathrm{Z} \rightarrow l^{-} l^{+}+X$}

In this section we describe our numerical setup and discuss the numerical results for the proton-(anti-)proton cross section $\sigma$ of the processes $\mathrm{pp} / \mathrm{p} \overline{\mathrm{p}} \rightarrow \gamma / \mathrm{Z} \rightarrow l^{-} l^{+}+X$ in the SM and the MSSM.

\subsection{Input parameters and setup for the SM}

The relevant SM input parameters are

$$
\begin{aligned}
& G_{\mu} \quad=1.16637 \times 10^{-5} \mathrm{GeV}^{-2} \\
& \alpha(0)=1 / 137.03599911, \quad \alpha\left(M_{\mathrm{Z}}\right)=1 / 128.93, \quad \alpha_{\mathrm{s}}\left(M_{\mathrm{Z}}\right)=0.1189, \\
& M_{\mathrm{W}, \mathrm{OS}}=80.403 \mathrm{GeV}, \quad M_{\mathrm{Z}, \mathrm{OS}}=91.1876 \mathrm{GeV}, \quad M_{\mathrm{H}}=115 \mathrm{GeV}, \\
& \Gamma_{\mathrm{W}, \mathrm{OS}}=2.141 \mathrm{GeV}, \quad \Gamma_{\mathrm{Z}, \mathrm{OS}}=2.4952 \mathrm{GeV}, \\
& m_{\mathrm{e}}=0.51099892 \mathrm{MeV}, \quad m_{\mu}=105.658369 \mathrm{MeV}, \quad m_{\tau} \quad=1.77699 \mathrm{GeV}, \\
& m_{\mathrm{u}}=66 \mathrm{MeV}, \quad m_{\mathrm{c}}=1.2 \mathrm{GeV}, \quad m_{\mathrm{t}}=174.2 \mathrm{GeV}, \\
& m_{\mathrm{d}}=66 \mathrm{MeV}, \quad m_{\mathrm{s}}=150 \mathrm{MeV}, \quad m_{\mathrm{b}}=4.6 \mathrm{GeV},
\end{aligned}
$$

which essentially follow ref. [117]. The masses of the light quarks are adjusted to reproduce the hadronic contribution to the photonic vacuum polarization of ref. [118]. The CKM matrix is set to unity. We keep finite light-quark masses in closed fermion loops, their numerical impact is, however, extremely small in the $\alpha\left(M_{\mathrm{Z}}\right)$ - and $G_{\mu}$-schemes. The $\mathcal{O}(\alpha)$ improved MRST2004qed set of PDF [98] is used throughout. If not stated otherwise, the QCD and QED factorization scales are identified and set to the Z-boson mass $M_{\mathrm{Z}}$.

\subsection{Phase-space cuts and event selection}

For the experimental identification of the processes $\mathrm{pp} / \mathrm{p} \overline{\mathrm{p}} \rightarrow \gamma / \mathrm{Z} \rightarrow l^{-} l^{+}+X$ we impose the set of phase-space cuts

$$
M_{l l}>50 \mathrm{GeV}, \quad p_{\mathrm{T}, l^{ \pm}}>25 \mathrm{GeV}, \quad\left|y_{l^{ \pm}}\right|<2.5,
$$

where $M_{l l}$ is the invariant mass of the dilepton system, $p_{\mathrm{T}, l^{ \pm}}$are the transverse momenta and $y_{l^{ \pm}}$the rapidities of the respective charged leptons. The cuts are not collinear safe with respect to the lepton momenta, so that observables in general receive corrections that 

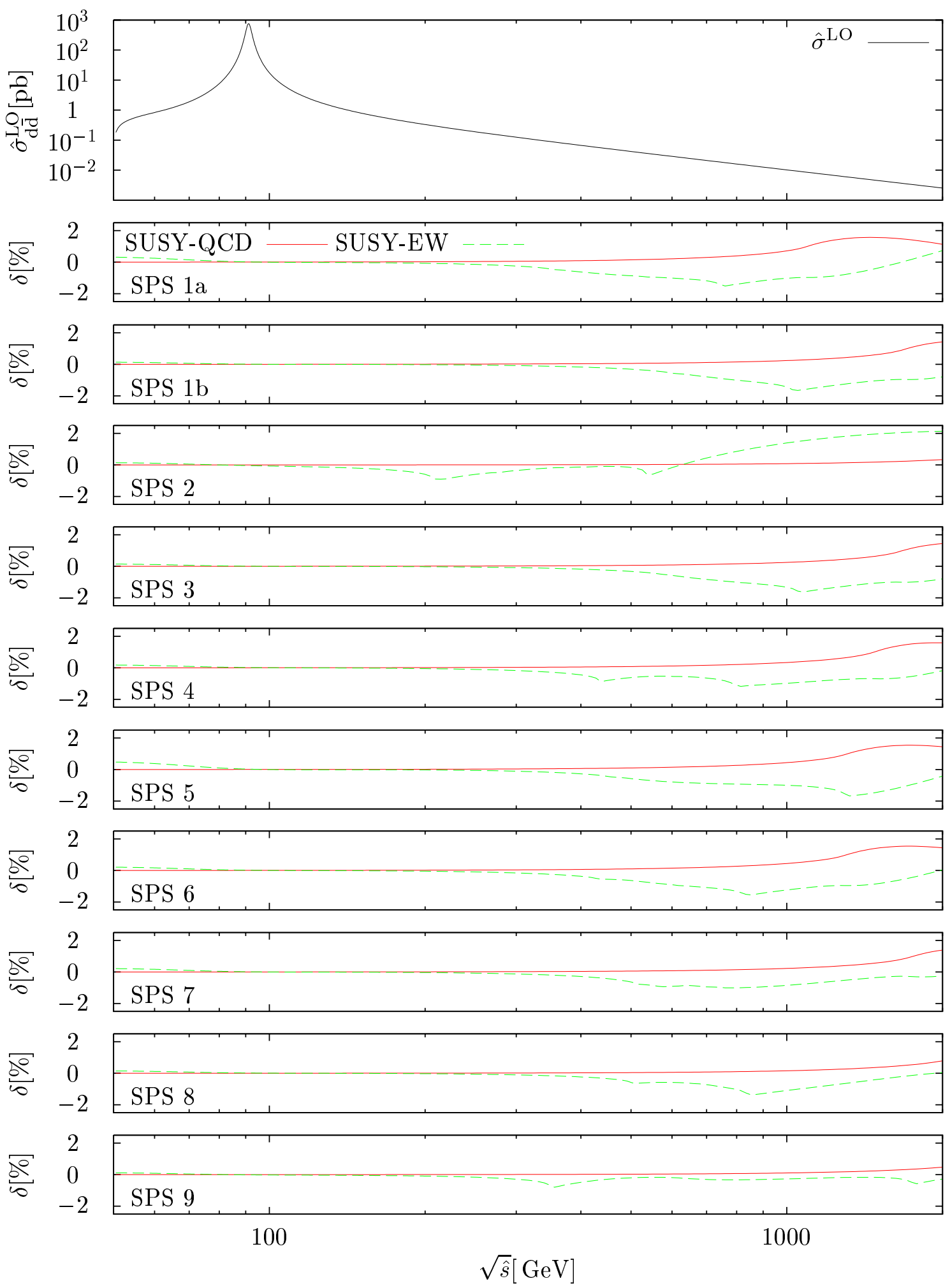

Figure 11. SUSY radiative corrections (MSSM $-\mathrm{SM})$ to the partonic process d $\overline{\mathrm{d}} \rightarrow l^{-} l^{+}$. 
involve large lepton-mass logarithms of the form $\alpha \ln \left(m_{l} / M_{\mathrm{Z}}\right)$. This is due to the fact that photons within a small collinear cone around the momenta of the leptons are not treated inclusively, i.e. the cuts assume perfect isolation of photons from the leptons. While this is (more or less) achievable for muon final states, it is not realistic for electrons. In order to be closer to the experimental situation for electrons, the following photon recombination procedure is applied:

1. Photons with a rapidity $\left|y_{\gamma}\right|>3$, which are close to the beams, are considered part of the proton remnant and are not recombined with the (anti-)lepton.

2. If the photon survived the first step, and if the resolution $R_{l^{ \pm} \gamma}=\sqrt{\left(y_{l^{ \pm}}-y_{\gamma}\right)^{2}+\phi_{l^{ \pm} \gamma}^{2}}$ is smaller than 0.1 (with $\phi_{l^{ \pm} \gamma}$ denoting the angles between the (anti-)lepton and the photon in the transverse plane), then the photon is recombined with the (anti-)lepton, i.e. the momenta of the photon and of the (anti-)lepton $l^{ \pm}$are added and associated with the momentum of $l^{ \pm}$, and the photon is discarded.

3. Finally, all events are discarded in which the resulting momentum of the (anti-)lepton does not pass the cuts given in (5.2).

The same recombination procedure was also used in ref. [42] for single-W production.

While the electroweak corrections differ for final-state electrons and muons without photon recombination, the corrections become universal in the presence of photon recombination, since the lepton-mass logarithms cancel in this case, in accordance with the KLN theorem. Numerical results are presented for photon recombination and for bare muons.

\subsection{SM predictions for cross sections at the LHC and the Tevatron}

In tables 1 and 2 we present the integrated LO cross section together with the electroweak and QCD correction factors $\delta_{a b}$ for the LHC with a centre-of-mass energy $\sqrt{s}=14 \mathrm{TeV}$ and for the Tevatron with $\sqrt{s}=1.96 \mathrm{TeV}$. The subscripts $a, b$ of the correction factors $\delta_{a b}$ denote the various partonic initial states for $\mathrm{pp} / \mathrm{p} \overline{\mathrm{p}}$ collisions. The results are given for six different ranges of the dilepton invariant mass $M_{l l}$.

By definition, our LO cross section $\sigma^{\mathrm{LO}}$ includes only contributions from $q \bar{q}$ initial states, i.e. we consistently treat all effects from photons in the initial state as corrections. To show the effect of the different treatments of the Z-boson resonance (see section 3.3) we give results for the LO cross section in the FS/PS schemes $\left.\sigma^{\mathrm{LO}}\right|_{\mathrm{FS} / \mathrm{PS}}$, which differ from the CMS only in the sub-permille range $(<0.01 \%)$. The $\gamma \gamma$-induced contribution to the LO cross section is given separately by the factor $\delta_{\gamma \gamma, 0}$. Apart from the suppression by the photon PDF, the partonic process $\gamma \gamma \rightarrow l^{-} l^{+}$does not involve a Z-boson resonance and therefore is strongly suppressed for low invariant mass $M_{l l}$. However, at the LHC for higher $M_{l l}$ the $\gamma \gamma$-induced contribution reaches up to $5-6 \%$ in our default setup. The $\mathcal{O}(\alpha)$ corrections $\delta_{\gamma \gamma \text {,phot }}$ and $\delta_{\gamma \gamma \text {,weak }}$ have very small effect on the integrated cross section at both the LHC and the Tevatron. In section 5.5 we shall pay particular attention to the question how an enhancement of effects of incoming photons may be achieved, a question that is interesting for a possible empirical fit of the photon PDF. 


$$
\mathrm{pp} \rightarrow l^{+} l^{-}+X \text { at } \sqrt{s}=14 \mathrm{TeV}
$$

\begin{tabular}{|c|c|c|c|c|c|c|}
\hline$M_{l l} / \mathrm{GeV}$ & $50-\infty$ & $100-\infty$ & $200-\infty$ & $500-\infty$ & $1000-\infty$ & $2000-\infty$ \\
\hline$\sigma_{0} / \mathrm{pb}$ & $738.733(6)$ & $32.7236(3)$ & $1.48479(1)$ & $0.0809420(6)$ & $0.00679953(3)$ & $0.000303744(1)$ \\
\hline$\left.\sigma_{0}\right|_{\mathrm{FS} / \mathrm{PS}} / \mathrm{pb}$ & $738.773(6)$ & $32.7268(3)$ & $1.48492(1)$ & $0.0809489(6)$ & $0.00680008(3)$ & $0.000303767(1)$ \\
\hline$\delta_{\gamma \gamma, 0} / \%$ & 0.17 & 1.15 & 4.30 & 4.92 & 5.21 & 6.17 \\
\hline$\delta_{q \bar{q}, \text { phot }}^{\mathrm{rec}} / \%$ & -1.81 & -4.71 & -2.92 & -3.36 & -4.24 & -5.66 \\
\hline$\delta_{q \bar{q}, \text { phot }}^{\mu^{+} \mu^{-}} / \%$ & -3.34 & -8.85 & -5.72 & -7.05 & -9.02 & -12.08 \\
\hline 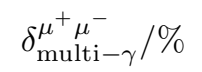 & $0.073_{-0.024}^{+0.027}$ & $0.49_{-0.15}^{+0.18}$ & $0.17_{-0.05}^{+0.06}$ & $0.23_{-0.06}^{+0.07}$ & $0.33_{-0.08}^{+0.09}$ & $0.54_{-0.12}^{+0.13}$ \\
\hline$\delta_{q \bar{q}, \text { weak }} / \%$ & -0.71 & -1.02 & -0.14 & -2.38 & -5.87 & -11.12 \\
\hline$\delta_{\text {h.o.weak }} / \%$ & 0.030 & 0.012 & -0.23 & -0.29 & -0.31 & -0.32 \\
\hline$\delta_{\text {Sudakov }}^{(2)} / \%$ & -0.00046 & -0.0067 & -0.035 & 0.23 & 1.14 & 3.38 \\
\hline$\delta_{q / \bar{q} \gamma, \text { phot }} / \%$ & -0.11 & -0.21 & 0.38 & 1.53 & 1.91 & 2.34 \\
\hline$\delta_{\gamma \gamma, \text { phot }}^{\mathrm{rec}} / \%$ & -0.0060 & -0.032 & -0.11 & -0.14 & -0.16 & -0.23 \\
\hline$\delta_{\gamma \gamma, \text { phot }}^{\mu^{+} \mu^{-}} / \%$ & -0.011 & -0.058 & -0.22 & -0.30 & -0.39 & -0.59 \\
\hline$\delta_{\gamma \gamma, \text { weak }} / \%$ & 0.000045 & 0.00056 & -0.025 & -0.14 & -0.31 & -0.64 \\
\hline$\delta_{\mathrm{QCD}} / \%$ & $4.0(1)$ & $13.90(6)$ & $26.10(3)$ & $21.29(2)$ & $8.65(1)$ & $-11.93(1)$ \\
\hline
\end{tabular}

Table 1. Integrated LO cross section and relative correction factors at the LHC for different values of the invariant mass cut $M_{l l}$.

For the photonic corrections we give results for bare muons $\left(\delta_{q \bar{q}, \text { phot }}^{\mu^{+} \mu^{-}}\right)$and with the recombination procedure described in the previous section $\left(\delta_{q \bar{q}, \text { phot }}^{\text {rec }}\right)$, where large logarithms $\propto \alpha \ln \left(\frac{m_{l}}{M_{\mathrm{Z}}}\right)$ cancel, so that the resulting corrections are smaller. The effect of higherorder final-state bremsstrahlung beyond $\mathcal{O}(\alpha)$, as described in section 3.4.3, is small for the integrated cross section, as $\delta_{\text {multi- }-\gamma}^{\mu^{+} \mu^{-}}$never exceeds the $0.1 \%$ level. However, as discussed below they become relevant for the invariant-mass distribution around the resonance. The correction $\delta_{\text {multi- }-\gamma}^{\mu^{+} \mu^{-}}$is given for the central scale choice $Q=\sqrt{\hat{s}}$ with an uncertainty estimate obtained from varying the scale $Q$ between $Q=3 \sqrt{\hat{s}}$ (upper number) and $Q=\sqrt{\hat{s}} / 3$ (lower number). Although the $q / \bar{q} \gamma$-induced photonic processes can be considered as being part of the $\mathcal{O}(\alpha)$ photonic corrections to the $q \bar{q}$-induced LO process, we do not include them in $\delta_{q \bar{q}, \text { phot }}$, but give them separately by $\delta_{q / \bar{q} \gamma, \text { phot }}$. They are small for all considered $M_{l l}$ ranges 
$\mathrm{p} \overline{\mathrm{p}} \rightarrow l^{+} l^{-}+X$ at $\sqrt{s}=1.96 \mathrm{TeV}$

\begin{tabular}{|c|c|c|c|c|c|c|}
\hline$M_{l l} / \mathrm{GeV}$ & $50-\infty$ & $100-\infty$ & $150-\infty$ & $200-\infty$ & $400-\infty$ & $600-\infty$ \\
\hline$\sigma_{0} / \mathrm{pb}$ & $142.7878(7)$ & $6.62280(3)$ & $0.824114(3)$ & $0.294199(1)$ & $0.01775063(5)$ & $0.001778465(5)$ \\
\hline$\left.\sigma_{0}\right|_{\mathrm{FS} / \mathrm{PS}} / \mathrm{pb}$ & $142.7948(7)$ & $6.62338(3)$ & $0.824183(3)$ & $0.294222(1)$ & $0.01775188(5)$ & $0.001778585(5)$ \\
\hline$\delta_{\gamma \gamma, 0} / \%$ & 0.15 & 0.72 & 1.54 & 1.44 & 0.83 & 0.57 \\
\hline$\delta_{q \bar{q}, \text { phot }}^{\mathrm{rec}} / \%$ & -1.85 & -4.87 & -3.65 & -3.83 & -5.16 & -6.56 \\
\hline$\delta_{q \bar{q}, \text { phot }}^{\mu^{+} \mu^{-}} / \%$ & -3.44 & -8.93 & -6.46 & -6.86 & -9.56 & -12.42 \\
\hline 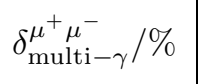 & $0.082_{-0.026}^{+0.032}$ & $0.48_{-0.15}^{+0.18}$ & $0.19_{-0.06}^{+0.07}$ & $0.20_{-0.06}^{+0.07}$ & $0.34_{-0.09}^{+0.10}$ & $0.55_{-0.14}^{+0.15}$ \\
\hline$\delta_{q \bar{q}, \text { weak }} / \%$ & -0.70 & -1.01 & 0.12 & 0.15 & -1.25 & -2.60 \\
\hline$\delta_{\text {h.o.weak }} / \%$ & 0.036 & -0.00094 & -0.23 & -0.29 & -0.35 & -0.36 \\
\hline$\delta_{\text {Sudakov }}^{(2)} / \%$ & -0.00014 & -0.00044 & 0.012 & 0.047 & 0.35 & 0.78 \\
\hline$\delta_{q / \bar{q} \gamma, \text { phot }} / \%$ & -0.070 & -0.14 & -0.16 & -0.063 & 0.090 & 0.15 \\
\hline$\delta_{\gamma \gamma, \text { phot }}^{\text {rec }} / \%$ & -0.0059 & -0.024 & -0.054 & -0.052 & -0.035 & -0.029 \\
\hline$\delta_{\gamma \gamma, \text { phot }}^{\mu^{+} \mu^{-}} / \%$ & -0.010 & -0.043 & -0.098 & -0.098 & -0.072 & -0.061 \\
\hline$\delta_{\gamma \gamma, \text { weak }} / \%$ & 0.000056 & 0.00081 & 0.0023 & -0.0038 & -0.012 & -0.014 \\
\hline$\delta_{\mathrm{QCD}} / \%$ & $14.19(7)$ & $18.07(4)$ & $19.15(1)$ & $17.72(1)$ & $9.47(1)$ & $1.48(1)$ \\
\hline
\end{tabular}

Table 2. Integrated LO cross section and relative correction factors at the Tevatron for different values of the invariant mass cut $M_{l l}$.

in our default setup, as expected from the suppression by a factor $\alpha$ and by the photon PDF.

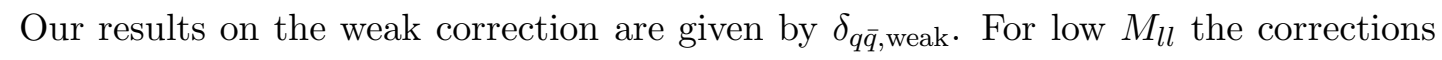
to the integrated cross section are of the order of a per cent. For the LHC at high invariant mass the weak corrections are enhanced due to large Sudakov logarithms, eventually getting of the same order as the photonic and QCD corrections. The smallness of the higher-order weak effects $\delta_{\text {h.o.weak }}$ and the leading two-loop Sudakov logarithms $\delta_{\text {Sudakov }}^{(2)}$, as described in sections 3.4.1 and 3.4.2, points towards the stability of the results concerning higher orders in $\alpha$, especially in the resonance region. Following the attitude of ref. [42] we consider the size of $\delta_{\text {Sudakov }}^{(2)}$ as a measure for the missing EW higher-order effects beyond NLO. For the LHC this estimate indicates a corresponding uncertainty at the level of $1-3 \%$ for invariant masses in the range of $1-2 \mathrm{TeV}$. For the Tevatron $\delta_{\text {Sudakov }}^{(2)}$ does not even reach the percent level up to invariant masses of $\sim 600 \mathrm{GeV}$, which suggests that EW effects beyond NLO 
do not significantly contribute to the theoretical uncertainty for Tevatron measurements.

The NLO QCD corrections $\delta_{\mathrm{QCD}}$ are evaluated for a fixed scale $\mu_{\mathrm{R}}=\mu_{\mathrm{F}}=M_{\mathrm{Z}}$ and vary strongly depending on the size of the cut on the dilepton invariant mass. The statistical error is somewhat larger for the QCD corrections since very large cancellations take place between the $q \bar{q}$ and the $q g / \bar{q} g$ induced channels.

\subsection{SM predictions for distributions at the LHC}

For brevity we restrict our investigation of distributions for the NC Drell-Yan process to the situation at the LHC. Already the results for integrated cross sections indicate that the relative corrections in the $\mathrm{Z}$ resonance region at the LHC and Tevatron are qualitatively very similar.

Figures 12, 13, and 14 show differential distributions and correction factors at the LHC. The uppermost plots show absolute distributions, followed below by the relative NLO corrections (QCD, photonic, and weak corrections) normalized to the $q \bar{q}$ LO result. Note that for some distributions the correction factors are rescaled. The plots at the bottom show the higher-order electroweak and photon-induced corrections. The distributions for $\sigma^{\mathrm{NLO}}$ and $\sigma^{\mathrm{NLO} \text {,rec }}$ are our best estimates for a $\mu^{+} \mu^{-}$final state and a recombined final state, respectively, and include all corrections shown in figures 12, 13, and 14.

In figure 12 we present the dilepton invariant-mass distribution $\mathrm{d} \sigma / \mathrm{d} M_{l l}$ in the resonance and the high-invariant-mass region at the LHC. The $M_{l l}$ distribution on the left shows the well-known large corrections due to the final-state photon bremsstrahlung which significantly change the shape of the resonance peak, since events belonging to the $\mathrm{Z}$ pole are shifted to smaller values of the invariant mass $M_{l l}$. Using photon recombination these corrections are somewhat smaller but still in the range of $40-45 \%$ at maximum. In the high-energy tail of the distribution, where the leptons are an important background for new physics searches, the photonic corrections rise in size up to the order of $-10 \%$. At $2 \mathrm{TeV}$ they are of the same generic size as the QCD and the genuinely weak corrections, which are enhanced due to large Sudakov logarithms at high energies. At the lower end of the invariant-mass distribution the QCD corrections reach $\approx-170 \%$ which demonstrates that the inclusion of QCD corrections to NLO only is insufficient there. In order to properly describe this end of the distribution, which is determined by the phase-space cuts, most probably QCD resummations are necessary - a task that is beyond the scope of this paper. The genuinely weak NLO corrections amount to some per cent in the resonance region and tend to negative values for increasing $M_{l l}$, reaching about $-10 \%$ at $M_{l l}=2 \mathrm{TeV}$. This effect is mainly due to the well-known EW Sudakov logarithms. The multi-photon final-state and photon-induced corrections around the resonance region are in the range of some per cent and thus comparable in size to the weak NLO corrections. In particular, the higher-order multi-photon final-state radiation reduces the effect of bremsstrahlung at resonance. The effect of universal weak corrections beyond NLO is very small over the whole $M_{l l}$ range. The photon-induced corrections are strongly suppressed at the $\mathrm{Z}$ pole, but reach the level of a few per cent away from the pole. As we observed for the integrated cross section, in the high-energy range the $\gamma \gamma$-induced processes contribute with $\sim 5 \%$ to $\sigma^{\mathrm{NLO}}$ in our 

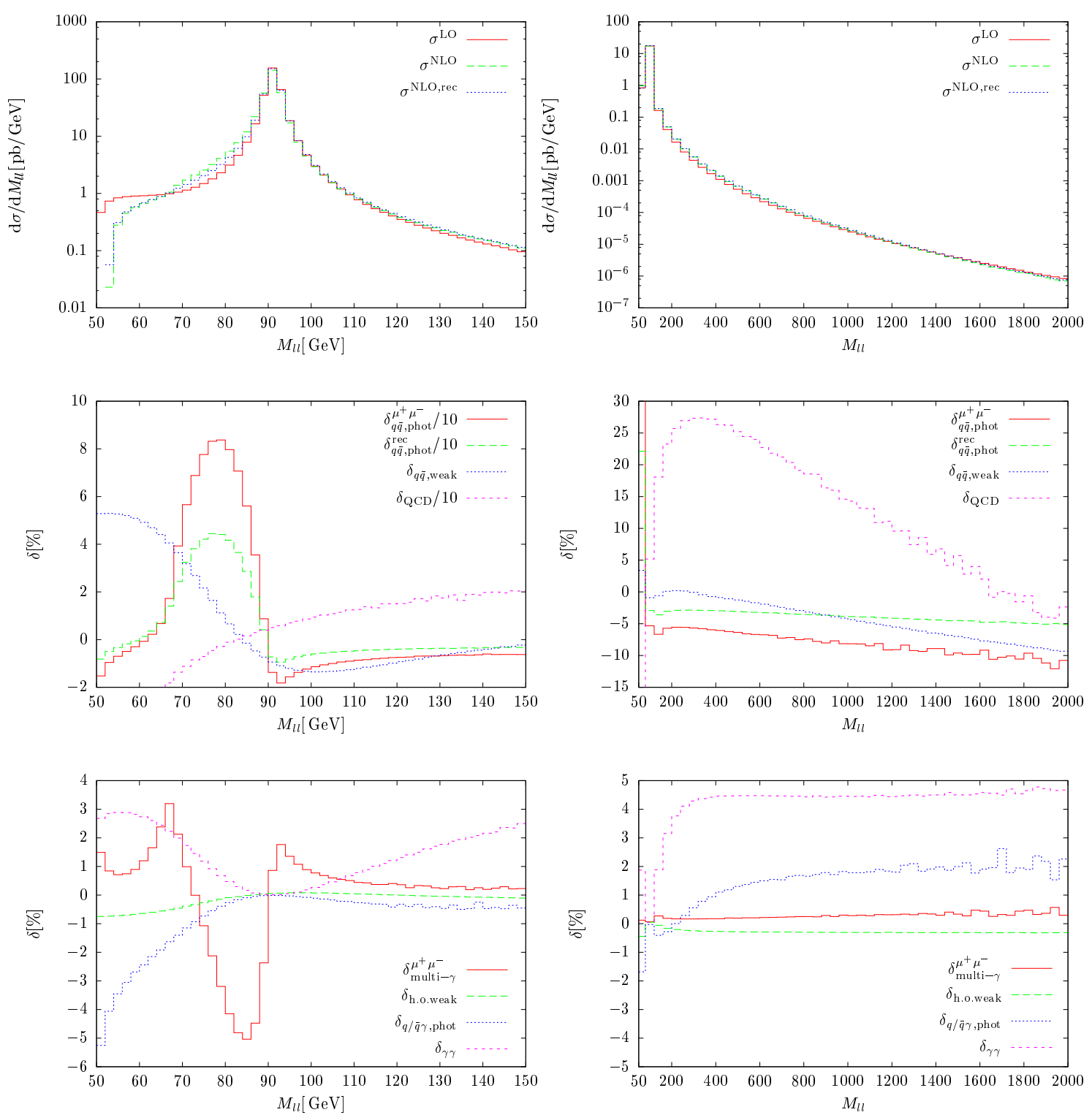

Figure 12. Dilepton invariant-mass distribution and correction factors at the LHC in the resonance region (left) and the high-invariant-mass region (right).

default setup, where not only the $\gamma \gamma$ LO contribution but also the corresponding photonic and weak corrections are included in the plots.

The lepton $p_{\mathrm{T}}$ distribution $\mathrm{d} \sigma / \mathrm{d} p_{\mathrm{T}, l^{-}}$is shown in figure 13 . The distribution has the well-known Jacobian peak at $p_{\mathrm{T}, l^{-}} \approx M_{\mathrm{Z}} / 2$. The EW corrections to the $p_{\mathrm{T}, l^{-}}$distribution are similar in shape to the CC case [27-32]. The photonic corrections, which are dominated by final-state radiation, distort the shape of the peak and are particularly sensitive to the fact whether photons are recombined with the outgoing leptons or not. The weak corrections are qualitatively similar to the $M_{l l}$ distribution, i.e. they are at the per-cent level and grow negative for increasing transverse momenta. As for the dilepton invariant- 

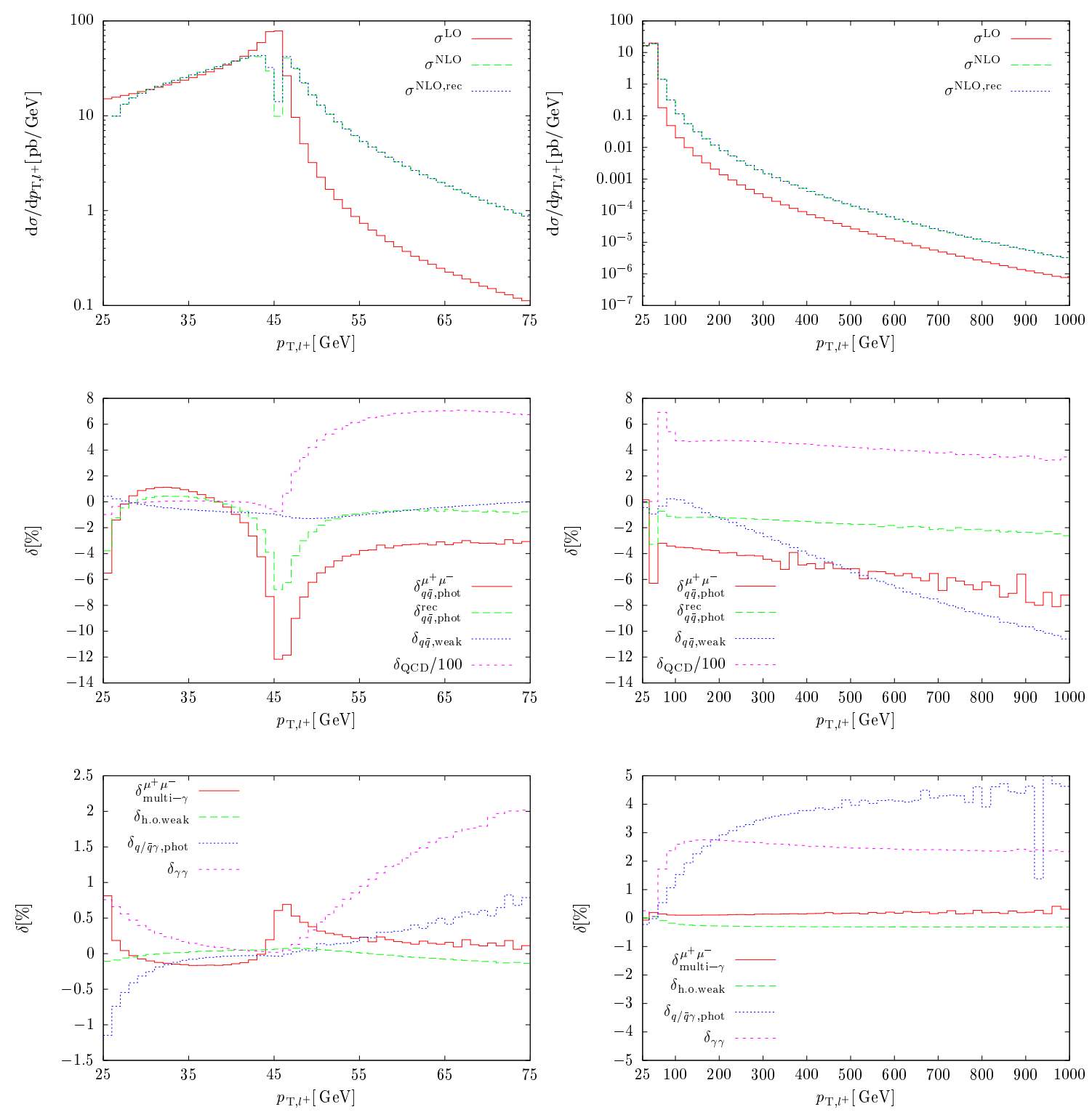

Figure 13. Lepton $p_{\mathrm{T}, l^{-}}$-distribution and correction factors at the LHC in the resonance region (left) and the high- $p_{\mathrm{T}}$ region (right).

mass distribution, close to the lower cut on $p_{\mathrm{T}, l^{-}}$the QCD corrections become negative and very large. In contrast to the $M_{l l}$ distribution, where the NLO QCD corrections show a moderate size away from the lower end point, the NLO QCD corrections to the $p_{\mathrm{T}}$ distribution are insufficient to describe the spectrum at all - an effect that is well-known in the literature. The reason for the dramatic rise of the QCD corrections for $p_{\mathrm{T}, l^{-}} \gtrsim M_{\mathrm{Z}} / 2$ lies in the fact that in $\mathrm{LO}$ the spectrum receives contributions from resonant $\mathrm{Z}$ bosons only for $p_{\mathrm{T}, l^{-}}<M_{\mathrm{Z}} / 2$, but in NLO resonant $\mathrm{Z}$ bosons also feed events into the range of larger $p_{\mathrm{T}}$ via the recoil of the $\mathrm{Z}$ boson against an emitted hard jet in the real corrections. A proper description of the transition between the two regions of $\mathrm{Z}$ and $\mathrm{Z}+$ jet production 

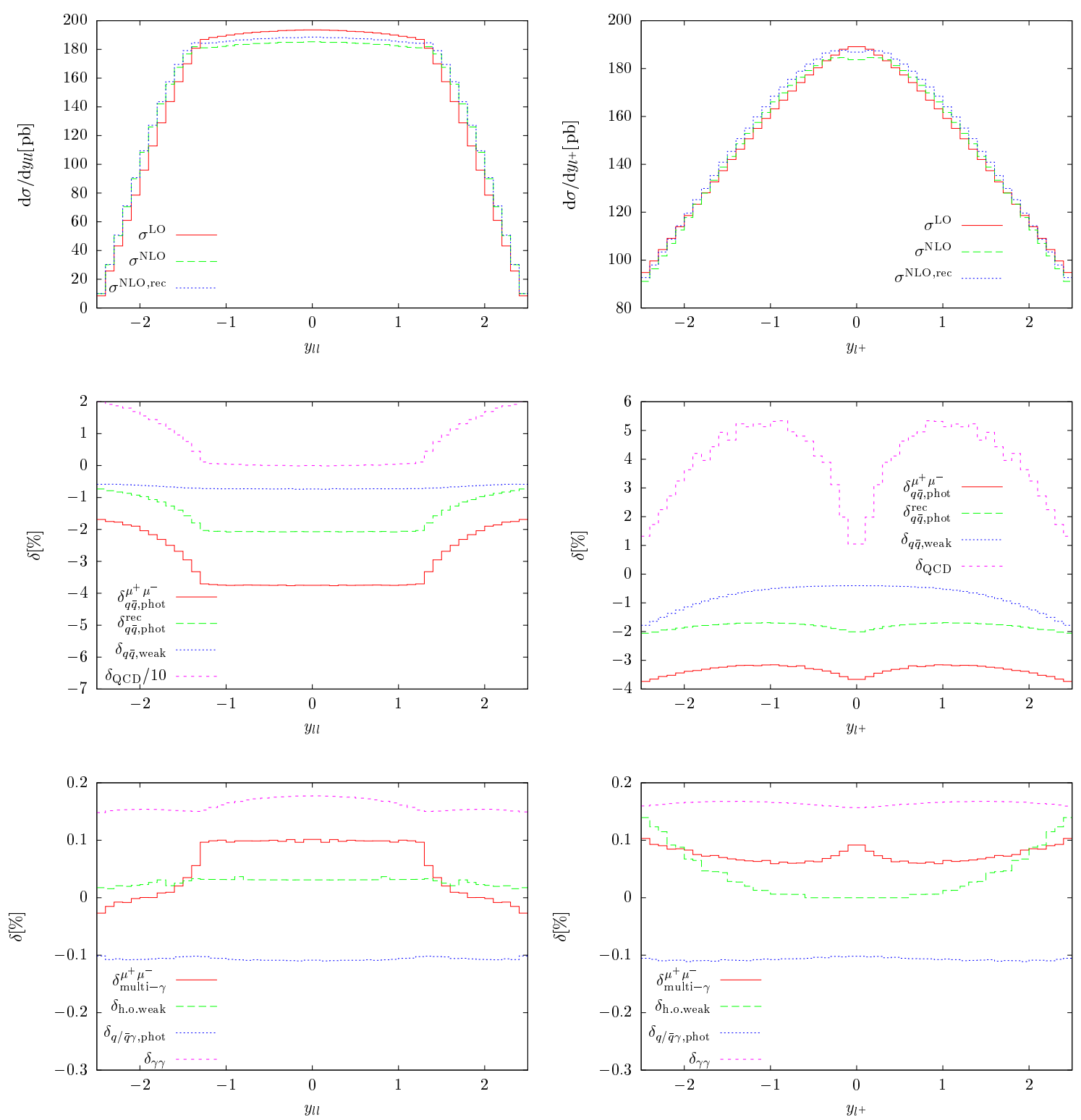

Figure 14. Dilepton (left) and lepton (right) rapidity distributions and correction factors at the LHC.

requires careful QCD resummations [16-26]. At $p_{\mathrm{T}, l^{-}} \approx M_{\mathrm{Z}} / 2$ the QCD corrections are of the order of $-75 \%$ and together with the negative EW corrections this leads to the dip we observe for $\sigma^{\mathrm{NLO}}$, and at high energies grow huge, reaching the level of several hundred per cent. The photon-induced corrections are again small at the peak related to the Z-boson resonance, but reach the level of a few per cent at very low and at high $p_{\mathrm{T}, l^{-}}$. The weak corrections beyond NLO are suppressed in the whole considered $p_{\mathrm{T}}$ region. The photonic corrections beyond $\mathcal{O}(\alpha)$ reduce the size of the NLO photonic corrections for very low $p_{\mathrm{T}, l^{-}}$ and at the resonance peak, but do not exceed $1 \%$ in size.

Figure 14 shows the dilepton and the lepton rapidity distributions $\mathrm{d} \sigma / \mathrm{d} y_{l l}$ and $\mathrm{d} \sigma / \mathrm{d} y_{l^{-}}$, 
respectively. The dilepton rapidity $y_{l l}$ is defined by

$$
y_{l l}=\frac{1}{2} \ln \left(\frac{k_{l l}^{0}+k_{l l}^{3}}{k_{l l}^{0}-k_{l l}^{3}}\right), \quad k_{l l}=k_{1}+k_{2} .
$$

All NLO QCD and EW corrections to the $y_{l l}$ distribution are at the level of few per cent, indicating the perturbative stability of this observable. This is confirmed by the extremely small size (below $0.2 \%$ ) of the higher-order EW effects shown in the lower left plot of figure 14. Qualitatively these statements also hold true for the distribution in the rapidity of the lepton, where the QCD corrections reach the $5 \%$ level.

\subsection{Enhancing effects of incoming photons by cuts}

In this section we study how the effect of photons in the initial state can be further enhanced by choosing different phase-space cuts. If the impact of incoming photons can be significantly extracted, dilepton production lends itself as a possible candidate for fitting the photon PDF. In this discussion, however, it is essential to include also QCD and photonic corrections, which are potentially large and especially sensitive to kinematical cuts. We consider the following three " $\gamma \gamma$ scenarios", which are defined by cuts in addition to our default setup, in order to enhance the effect of incoming photons:

(a) $p_{\mathrm{T}, l^{ \pm}}<M_{l l} / 4$.

This cut is motivated by the consideration that $\gamma \gamma$ fusion involves $t$ - and $u$-channel diagrams, while the $q \bar{q}$ annihilation proceeds via $s$-channel diagrams only, i.e. $\gamma \gamma$ fusion prefers a small value of $\sin \theta^{*}$, with $\theta^{*}$ denoting the scattering angle of the lepton in the partonic centre-of-mass frame, while $q \bar{q}$ annihilation favours intermediate angles. In LO we have $M_{l l}=\sqrt{\hat{s}}$ and $p_{\mathrm{T}, l^{ \pm}}=\frac{1}{2} \sqrt{\hat{s}} \sin \theta^{*}=\frac{1}{2} M_{l l} \sin \theta^{*}$, i.e. the above cut translates into $\sin \theta^{*}<\frac{1}{2}$.

(b) $p_{\mathrm{T}, l^{ \pm}}<50 \mathrm{GeV}$.

Following the same considerations this cut translates into the condition $\sin \theta^{*}<$ $100 \mathrm{GeV} / M_{l l}$ for the LO contribution. For increasing invariant masses $M_{l l}$ only smaller and smaller scattering angles are included.

(c) $p_{\mathrm{T}, l^{ \pm}}<100 \mathrm{GeV}$.

This case is similar to (b), but with the more relaxed LO condition $\sin \theta^{*}<$ $200 \mathrm{GeV} / M_{l l}$.

In table 3 we present the integrated LO cross section and the photon-induced processes as well as the photonic and QCD corrections for the three scenarios. The corresponding dilepton invariant-mass distributions and the same types of corrections are shown in figure 15, where the bands around the central lines correspond to a variation of the renormalization and factorization scale in the range $M_{\mathrm{Z}} / 2<\mu_{\mathrm{R}}=\mu_{\mathrm{F}}<2 M_{\mathrm{Z}}$.

The Z-resonance region is clearly dominated by $q \bar{q}$ annihilation and therefore not suited to access effects from photonic initial states. Scenario (a) excludes this region by the applied cut completely, since the lower cut on $p_{\mathrm{T}, l^{ \pm}}$implies $M_{l l}>100 \mathrm{GeV}$, so that the 
$\gamma \gamma$ scenario (a):

$\mathrm{pp} \rightarrow l^{+} l^{-}+X$ at $\sqrt{s}=14 \mathrm{TeV}$

\begin{tabular}{|c|c|c|c|c|}
\hline$M_{l l} / \mathrm{GeV}$ & $100-\infty$ & $150-\infty$ & $200-\infty$ & $250-\infty$ \\
\hline$\sigma^{\mathrm{LO}} / \mathrm{pb}$ & $0.91070(6)$ & $0.28175(1)$ & $0.116082(5)$ & $0.058598(2)$ \\
\hline$\delta_{\gamma \gamma, 0} / \%$ & 6.90 & 13.45 & 16.93 & 18.48 \\
\hline$\delta_{\gamma \gamma, \text { phot }}^{\text {rec }} / \%$ & -0.39 & -0.63 & -0.71 & -0.74 \\
\hline$\delta_{\gamma \gamma, \text { phot }}^{\mu^{+} \mu^{-}} / \%$ & -0.65 & -1.06 & -1.25 & -1.34 \\
\hline$\delta_{q / \bar{q} \gamma, \text { phot }} / \%$ & $-4.55(1)$ & $-5.79(2)$ & $-4.68(2)$ & $-3.12(3)$ \\
\hline$\delta_{q \bar{q}, \text { phot }}^{\text {rec }} / \%$ & $-10.06(2)$ & -7.64 & $-7.28(1)$ & -7.23 \\
\hline$\delta_{q \bar{q}, \text { phot }}^{\mu^{+} \mu^{-}} / \%$ & $-15.28(2)$ & -11.49 & -11.14 & -11.28 \\
\hline$\delta_{\mathrm{QCD}} / \%$ & $-121.2(5)$ & $-51.0(1)$ & $-35.8(1)$ & $-29.1(1)$ \\
\hline
\end{tabular}

$\gamma \gamma$ scenario $(\mathrm{b})$ :

\begin{tabular}{|l|ccccc|}
\multicolumn{7}{|c|}{$\mathrm{pp} \rightarrow l^{+} l^{-}+X$ at $\sqrt{s}=14 \mathrm{TeV}$} \\
\hline$M_{l l} / \mathrm{GeV}$ & $50-\infty$ & $100-\infty$ & $150-\infty$ & $200-\infty$ & $250-\infty$ \\
\hline$\sigma^{\mathrm{LO}} / \mathrm{pb}$ & $723.28(1)$ & $17.2883(5)$ & $0.37205(2)$ & $0.052388(3)$ & $0.011037(1)$ \\
$\delta_{\gamma \gamma, 0} / \%$ & 0.15 & 1.08 & 9.98 & 20.39 & 31.62 \\
$\delta_{\gamma \gamma, \mathrm{phot}}^{\mathrm{rec}} / \%$ & -0.0059 & -0.046 & -0.46 & -0.99 & -1.60 \\
$\delta_{\gamma \gamma, \mathrm{phot}}^{\mu^{+} \mu^{-}} / \%$ & -0.011 & -0.081 & -0.78 & -1.65 & -2.65 \\
$\delta_{q / \bar{q} \gamma, \mathrm{phot}} / \%$ & -0.13 & -0.66 & $-4.92(2)$ & $-8.86(7)$ & $-12.8(1)$ \\
$\delta_{q \bar{q}, \mathrm{phot}}^{\mathrm{rec}} / \%$ & -1.91 & -6.79 & -6.97 & $-8.09(1)$ & $-9.14(2)$ \\
$\delta_{q \bar{q}, \mathrm{phot}}^{\mu^{+} \mu^{-}} / \%$ & -3.46 & -12.33 & -10.69 & $-11.75(1)$ & $-12.89(2)$ \\
$\delta_{\mathrm{QCD}} / \%$ & $-20.4(1)$ & $-47.9(1)$ & $-45.5(2)$ & $-51.1(2)$ & $-58.2(6)$ \\
\hline
\end{tabular}

$\gamma \gamma$ scenario $(\mathrm{c})$ :

\begin{tabular}{|l|ccccc|}
\multicolumn{7}{|c|}{$\mathrm{pp} \rightarrow l^{+} l^{-}+X$ at $\sqrt{s}=14 \mathrm{TeV}$} \\
\hline$M_{l l} / \mathrm{GeV}$ & $50-\infty$ & $100-\infty$ & $150-\infty$ & $200-\infty$ & $250-\infty$ \\
\hline$\sigma^{\mathrm{LO}} / \mathrm{pb}$ & $737.827(6)$ & $31.8101(3)$ & $2.97905(5)$ & $0.57044(1)$ & $0.130466(6)$ \\
$\delta_{\gamma \gamma, 0} / \%$ & 0.17 & 1.11 & 3.80 & 6.68 & 11.21 \\
$\delta_{\gamma \gamma, \mathrm{phot}}^{\text {rec }} / \%$ & -0.0060 & -0.033 & -0.12 & -0.24 & -0.41 \\
$\delta_{\gamma \gamma, \text { phot }}^{\mu^{+} \mu^{-}} / \%$ & -0.011 & -0.059 & -0.23 & -0.45 & -0.76 \\
$\delta_{q / \bar{q} \gamma, \text { phot }} / \%$ & -0.11 & -0.31 & -0.81 & $-1.27(1)$ & $-1.50(1)$ \\
$\delta_{q \bar{q}, \text { phot }}^{\text {rec }} / \%$ & -1.81 & -4.85 & -3.87 & -5.29 & -5.95 \\
$\delta_{q \bar{q}, \text { phot }}^{\mu^{+} \mu^{-}} / \%$ & -3.34 & -9.11 & -7.30 & -9.67 & -10.03 \\
$\delta_{\mathrm{QCD}} / \%$ & $3.0(1)$ & $9.30(6)$ & $1.46(6)$ & $-19.2(1)$ & $-17.5(1)$ \\
\hline
\end{tabular}

Table 3. LO cross section from $q \bar{q}$ annihilation together with the relative impact from $\gamma \gamma$ and $q / \bar{q} \gamma$ initial states, as well as from photonic and QCD corrections, evaluated in the three different $\gamma \gamma$ scenarios (a), (b), and (c) as described in the text. 
$\gamma \gamma$ scenario (a):
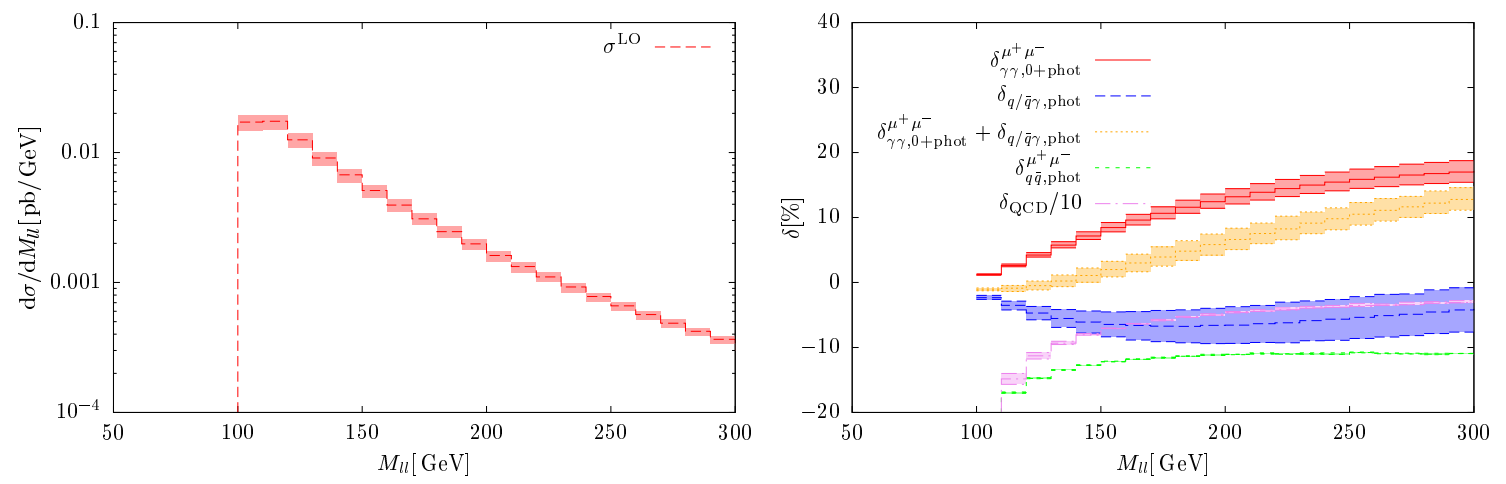

$\gamma \gamma$ scenario (b):
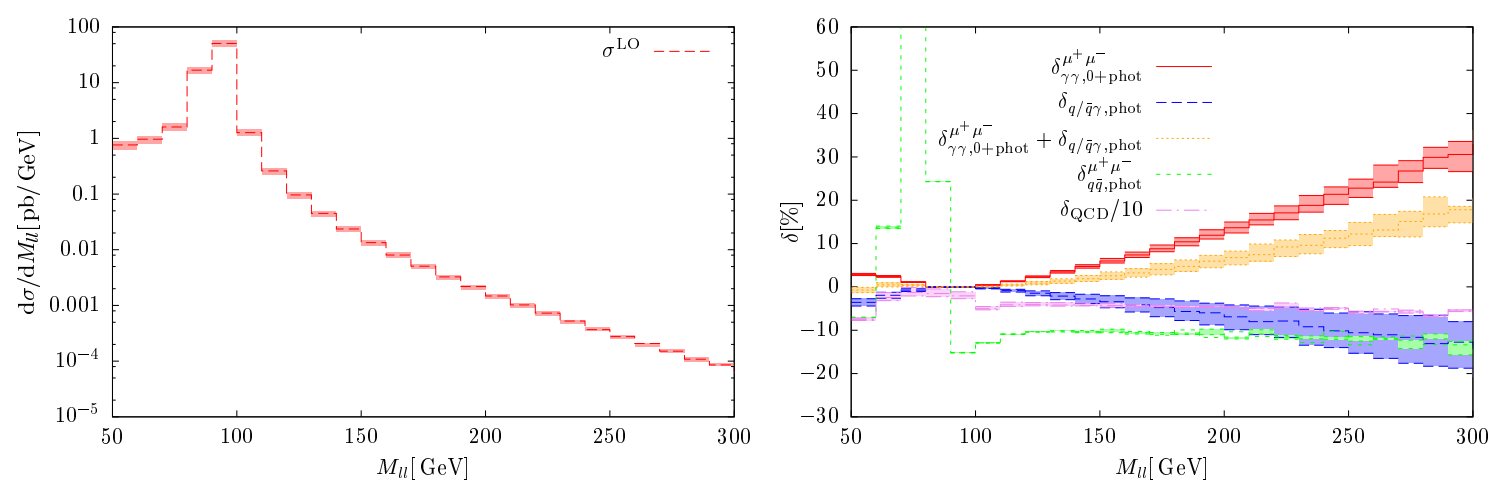

$\gamma \gamma$ scenario $(c)$ :
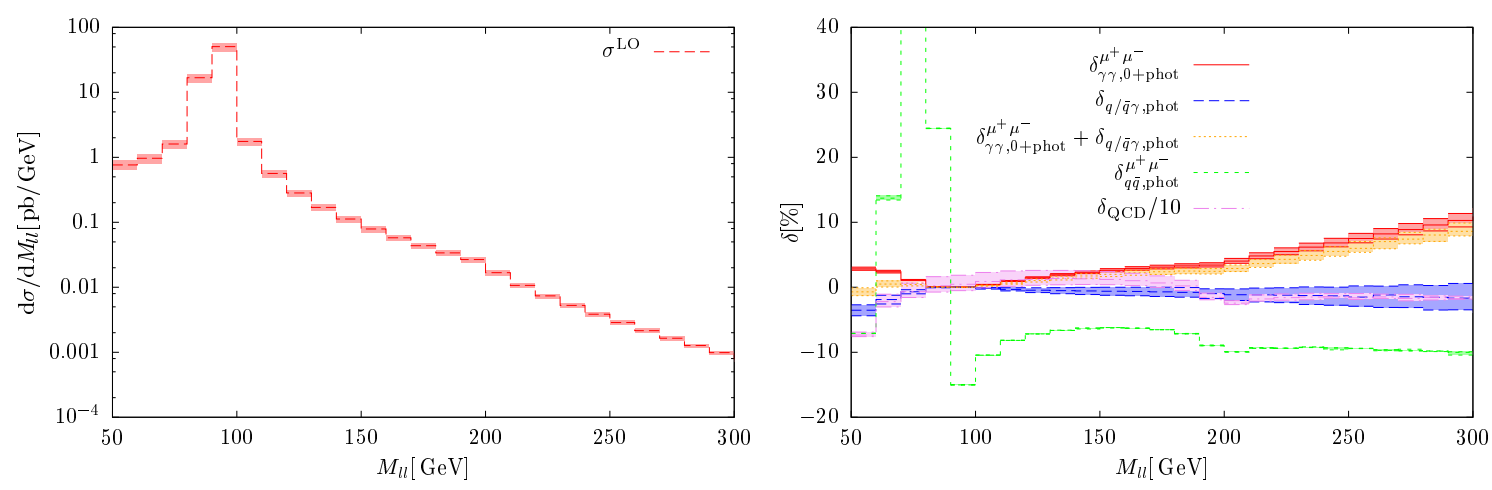

Figure 15. Dilepton invariant-mass distribution and correction factors at the LHC for the various $\gamma \gamma$ scenarios (a), (b), and (c), as described in the text. Within the bands the scale $\mu=\mu_{\mathrm{F}}=\mu_{\mathrm{R}}$ is varied in the range $M_{\mathrm{Z}} / 2<\mu<2 M_{\mathrm{Z}}$.

first two columns of numbers in table 3 are equal. Phenomenologically these two columns are useless, since "edge-of-phase-space effects" render QCD corrections unphysically large there. Scenarios (b) and (c) fully include the $\mathrm{Z}$ peak in the first column of numbers in table 3 , and a significant difference between these two scenarios is visible only in the QCD corrections. This is also trivially visible in figure 15. The interesting region for studying photonic initial states is the one of higher invariant masses $M_{l l}$, viz. $M_{l l} \gtrsim 150 \mathrm{GeV}$. In 
scenarios (a) and (b) already for $M_{l l} \gtrsim 150-200 \mathrm{GeV}$ the impact of $\gamma \gamma$ initial states reaches the order of 10-20\%; in case (c) it is still 4-7\%. In scenarios (a) and (b) it should, however, be realized that the effect of $\gamma \gamma$ initial states is systematically reduced by some per cent by the contribution of $q / \bar{q} \gamma$ initial states. The correlation between $\gamma \gamma$ and $q / \bar{q} \gamma$ initial states is also visible in the fact that the sum of the two shows a somewhat smaller sensitivity to the variation of the factorization scale than the two individual contributions, as seen in the distributions of figure 15. In view of the smallness of the integrated cross sections, which are of the order of $0.01 \mathrm{pb}$ to $1 \mathrm{pb}$ depending on the $M_{l l}$ range and the chosen scenario, it is not clear whether a fit of the photon PDF within a reasonable accuracy will be feasible. This possibility has to be analyzed in a dedicated study that carefully takes into account all experimental and theoretical uncertainties, in particular, from higher-order QCD effects. The QCD corrections, which are given in NLO in table 3 and figure 15, are of the order of $-30 \%$ to $-50 \%$ and thus quite large; the fact that they are negative, however, helps to further enhance the impact of photonic in initial states. The photonic corrections to $q \bar{q}$ initial states are about $-10 \%$ and thus go into the same direction as well, but the main uncertainty will certainly come from QCD corrections. The photonic corrections, which are included in $\delta_{\gamma \gamma, 0+\text { phot }}^{\mu^{+} \mu^{-}}$, and the weak (not explicitly shown here) corrections to the $\gamma \gamma$ initial states will not play a role in this context. In view of the overall size of the cross sections and the sizes of the $\gamma \gamma$ contributions and the QCD corrections, $\gamma \gamma$ scenario (a) seems the most promising to access the photon initial states.

\subsection{Comparison to SM results of other groups}

In order to make contact to results previously presented in the literature [33, 34, 36, 37], we have added our results on integrated cross sections and NLO EW corrections to the tuned comparison shown in table 2 of ref. [39]. In detail, for this comparison we conformed our input to the setup of "bare cuts" described there. Table 4, which shows our results together with the ones obtained with the HORACE [36], SANC [37], and ZGRAD2 [33, 34] programs, reveals good agreement between the various calculations. The remaining differences, which are at the $0.1 \%$ level, are phenomenologically irrelevant and should be due to slightly different settings in the programs, such as the treatment of small fermion masses.

In order to demonstrate the agreement of our results on distributions with previously published results, a comparison of the genuine NLO EW and multi-photon corrections for various distributions to results obtained with HORACE is shown in figures 16, 17, and 18.

In this comparison the HORACE results and the complete numerical setup and input are taken over from ref. [36]. There is very good agreement for the genuine $\mathcal{O}(\alpha)$ corrections, as it should be, because these corrections are defined exactly in the same way in the two calculations. Even the multi-photon corrections perfectly agree, although HORACE employs a parton-shower approach for their modelling, while we use structure functions for collinear multi-photon radiation. The band defining our result in figure 16 indicates the effect from varying the QED scale (3.58) by a factor 3 up and down. A similar comparison in the case of the CC Drell-Yan process was performed in ref. [42], revealing agreement between the two approaches at a somewhat lower level of accuracy. 


\begin{tabular}{|c|c|c|c|}
\hline \multicolumn{4}{|c|}{ LHC, $\mathrm{p} \overline{\mathrm{p}} \rightarrow Z, \gamma \rightarrow e^{+} e^{-}$} \\
\hline & $\mathrm{LO}[\mathrm{pb}]$ & NLO [pb] & $\delta[\%]$ \\
\hline HORACE & $739.34(3)$ & $742.29(4)$ & $0.40(1)$ \\
\hline $\mathrm{SANC}$ & $739.3408(3)$ & $743.072(7)$ & $0.504(1)$ \\
\hline ZGRAD2 & $737.8(7)$ & $743.0(7)$ & $0.71(9)$ \\
\hline our results & $739.343(1)$ & $742.68(1)$ & $0.451(1)$ \\
\hline \multicolumn{4}{|c|}{ LHC, $\mathrm{p} \overline{\mathrm{p}} \rightarrow Z, \gamma \rightarrow \mu^{+} \mu^{-}$} \\
\hline & $\mathrm{LO}[\mathrm{pb}]$ & NLO [pb] & $\delta[\%]$ \\
\hline HORACE & $739.33(3)$ & $762.20(3)$ & $3.09(1)$ \\
\hline SANC & $739.3355(3)$ & $762.645(3)$ & $3.1527(4)$ \\
\hline ZGRAD2 & $740(1)$ & $764(1)$ & $3.2(2)$ \\
\hline our results & $739.343(1)$ & $762.21(1)$ & $3.092(1)$ \\
\hline
\end{tabular}

Table 4. Extension of the tuned comparison shown in table 2 of ref. [39] for "bare cuts".

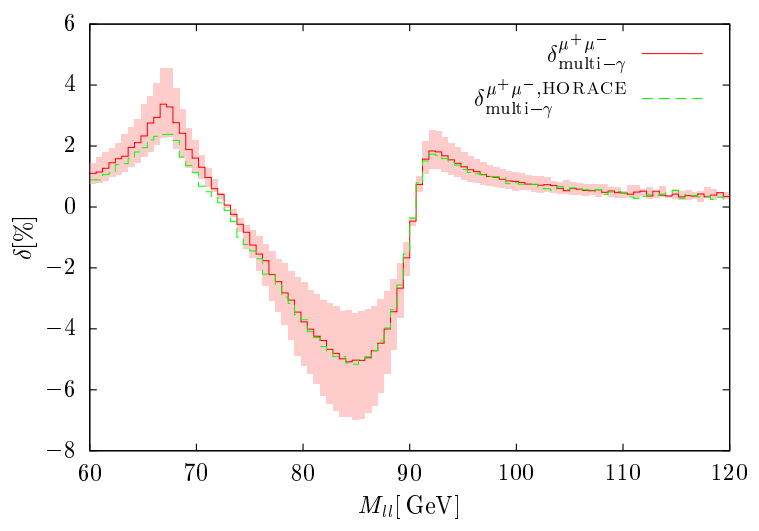

Figure 16. Comparison of the multi-photon corrections for the dilepton invariant-mass distribution to results obtained with HORACE.

\subsection{Numerical results on supersymmetric corrections in the MSSM}

Our discussion closely follows the one presented in ref. [42] for the CC case of single-W production. We choose the SM input parameters and the setup of the calculation (input parameter scheme, PDF, cuts, etc.) as described in section 5.1 and study the dependence of the corrections on the SUSY breaking parameters by considering all the SPS benchmark scenarios [119]. Both for the CC and NC case, the generic suppression of the genuine SUSY corrections is insensitive to a specific scenario. We therefore refrain from further restricting the SPS coverage by taking into account recent experimental bounds in favour of a broader scope in the SUSY parameter space. The SPS points are defined by the lowenergy SUSY breaking parameters which determine the spectrum and the couplings. For the ten benchmark scenarios under consideration in ref. [42] and in this work, this input ${ }^{5}$ is also tabulated in appendix $\mathrm{C}$.

\footnotetext{
${ }^{5}$ See The Snowmass Points and Slopes (SPS) homepage, http://www.cpt.dur.ac.uk/ georg/sps/.
} 

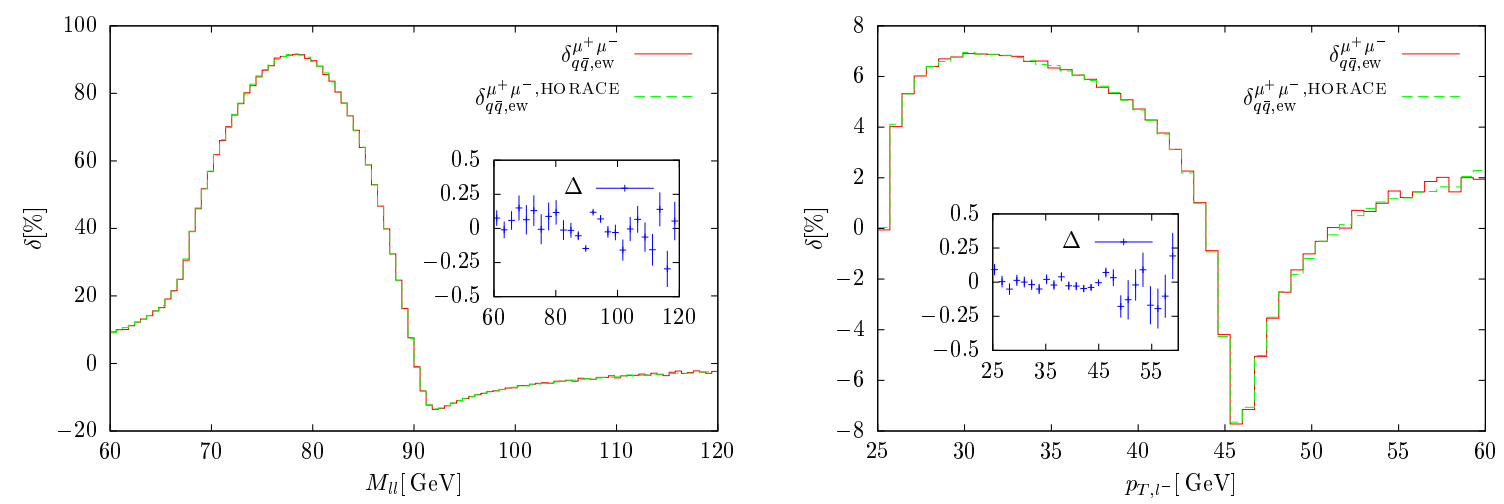

Figure 17. Comparison of the NLO EW corrections for the dilepton invariant-mass (left) and the $p_{\mathrm{T}, l^{-}}$(right) distributions to results obtained with HORACE, with $\Delta$ showing the difference of the two results on the relative correction $\delta$ in the inset, where the HORACE integration error defines the error bars.
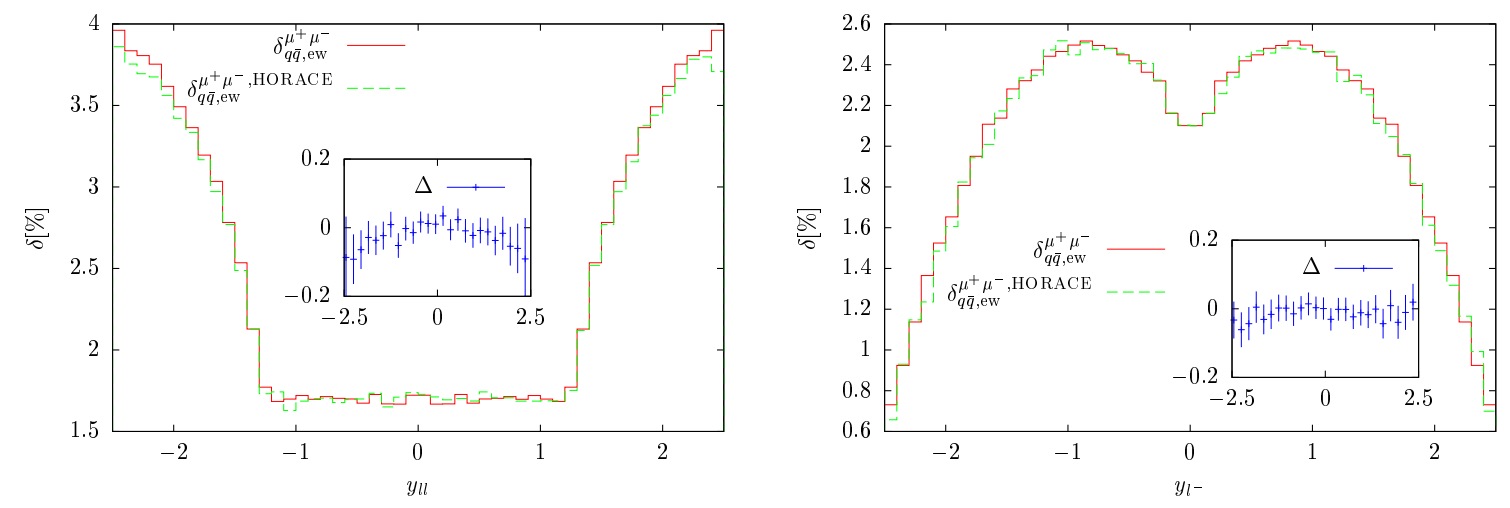

Figure 18. As in figure 17, but for the dilepton (left) and lepton rapidity (right) distributions.

Dependent SUSY parameters, such as Higgs, chargino, neutralino, or sfermion masses, are calculated from the SPS input using tree-level relations. Since the impact of the fermion masses of the first two generations is negligible, these masses are set to zero in the calculation of the corresponding sfermion mass matrices. Following this approach the SUSY corrections do not depend on the lepton generations in the partonic process $q \bar{q} \rightarrow l^{-} l^{+}$, i.e. the SUSY corrections presented below are valid both for outgoing electrons and muons.

In table 5 we list our results for the SUSY corrections within the MSSM at the LHC. The corresponding LO cross sections can be found in table 1. We give results for SUSYQCD and SUSY-EW corrections separately as described in section 4. As expected and similar to the CC case [42], the corrections for low invariant dilepton mass $M_{l l}$ are negligible at the level of $0.1 \%$ or below. Only for very high $M_{l l}$ and only for a few scenarios the corrections reach the level of $1-2 \%$. Similar to the CC case the maximum is reached for the SPS2 scenario where the gauginos are particularly light and the squarks and sleptons are so heavy that their negative contribution becomes effective only at higher invariant mass. Table 6 essentially shows the same features for the situation at Tevatron (for LO numbers see table 2), where the SUSY corrections for the highest reachable invariant masses 


$$
\mathrm{pp} \rightarrow l^{+} l^{-}+X \text { at } \sqrt{s}=14 \mathrm{TeV}
$$

\begin{tabular}{|cc|cccccc|}
\hline \multicolumn{2}{|c}{$M_{\text {ll }} / \mathrm{GeV}$} & $50-\infty$ & $100-\infty$ & $200-\infty$ & $500-\infty$ & $1000-\infty$ & $2000-\infty$ \\
\hline SPS1a & $\delta_{\text {SUSY-EW }} / \%$ & $0.0094(3)$ & $-0.0041(1)$ & -0.053 & -0.43 & -0.33 & 0.73 \\
SPS1a & $\delta_{\text {SUSY-QCD }} / \%$ & 0.0060 & 0.012 & 0.062 & 0.34 & 1.19 & 0.61 \\
\hline SPS1b & $\delta_{\text {SUSY-EW }} / \%$ & $0.0076(1)$ & 0.0021 & -0.018 & -0.31 & -0.67 & -0.20 \\
SPS1b & $\delta_{\text {SUSY-QCD }} / \%$ & 0.0025 & 0.0049 & 0.025 & 0.13 & 0.48 & 1.38 \\
\hline SPS2 & $\delta_{\text {SUSY-EW }} / \%$ & -0.046 & -0.12 & -0.40 & 0.30 & 1.60 & 1.94 \\
SPS2 & $\delta_{\text {SUSY-QCD }} / \%$ & 0.00093 & 0.0018 & 0.0091 & 0.045 & 0.15 & 0.58 \\
\hline SPS3 & $\delta_{\text {SUSY-EW }} / \%$ & $0.0046(1)$ & $-0.00072(7)$ & -0.021 & -0.32 & -0.66 & -0.20 \\
SPS3 & $\delta_{\text {SUSY-QCD }} / \%$ & 0.0026 & 0.0050 & 0.026 & 0.14 & 0.50 & 1.37 \\
\hline SPS4 & $\delta_{\text {SUSY-EW }} / \%$ & 0.013 & $0.0005(1)$ & -0.061 & -0.24 & -0.24 & 0.27 \\
SPS4 & $\delta_{\text {SUSY-QCD }} / \%$ & 0.0034 & 0.0066 & 0.035 & 0.18 & 0.68 & 1.30 \\
\hline SPS5 & $\delta_{\text {SUSY-EW }} / \%$ & 0.025 & 0.013 & 0.063 & -0.23 & -0.51 & 0.11 \\
SPS5 & $\delta_{\text {SUSY-QCD }} / \%$ & 0.0042 & 0.0083 & 0.043 & 0.23 & 0.85 & 1.05 \\
\hline SPS6 & $\delta_{\text {SUSY-EW }} / \%$ & 0.010 & $0.0004(2)$ & -0.042 & -0.42 & -0.44 & 0.32 \\
SPS6 & $\delta_{\text {SUSY-QCD }} / \%$ & 0.0042 & 0.0082 & 0.043 & 0.23 & 0.85 & 1.05 \\
\hline SPS7 & $\delta_{\text {SUSY-EW }} / \%$ & $0.0094(2)$ & $0.0002(2)$ & -0.049 & -0.42 & -0.032 & 0.23 \\
SPS7 & $\delta_{\text {SUSY-QCD }} / \%$ & 0.0023 & 0.0046 & 0.024 & 0.12 & 0.45 & 1.40 \\
\hline SPS8 & $\delta_{\text {SUSY-EW }} / \%$ & 0.012 & 0.0039 & -0.035 & -0.34 & -0.17 & 0.22 \\
SPS8 & $\delta_{\text {SUSY-QCD }} / \%$ & 0.0017 & 0.0033 & 0.017 & 0.088 & 0.31 & 1.26 \\
\hline SPS9 & $\delta_{\text {SUSY-EW }} / \%$ & -0.015 & -0.026 & -0.11 & 0.029 & 0.095 & $-0.0059(1)$ \\
SPS9 & $\delta_{\text {SUSY-QCD }} / \%$ & 0.0012 & 0.0023 & 0.012 & 0.059 & 0.20 & 0.82 \\
\hline
\end{tabular}

Table 5. Relative SUSY-QCD and SUSY-EW correction factors at the LHC for different values of the invariant-mass cut $M_{l l}$ for the SPS benchmark scenarios. The corresponding LO cross sections can be found in table 1.

are even smaller compared to the LHC.

In figure 19 we show the invariant-mass distribution $\mathrm{d} \sigma / \mathrm{d} M_{l l}$ for the LHC. As already observed for the integrated cross section, the distributions receive per-cent corrections only for an invariant mass in the $\mathrm{TeV}$ range. The maximum correction is again found for SPS2 


\begin{tabular}{|cc|cccccc|}
\multicolumn{7}{c}{$\mathrm{p} \overline{\mathrm{p}} \rightarrow l^{+} l^{-}+X$ at $\sqrt{s}=1.96 \mathrm{TeV}$} \\
\hline \multicolumn{2}{|c|}{$M_{\text {ll }} / \mathrm{GeV}$} & $50-\infty$ & $100-\infty$ & $150-\infty$ & $200-\infty$ & $400-\infty$ & $600-\infty$ \\
\hline SPS1a & $\delta_{\text {SUSY-EW }} / \%$ & $0.0022(2)$ & $0.0034(1)$ & 0.052 & 0.032 & -0.14 & -0.26 \\
SPS1a & $\delta_{\text {SUSY-QCD }} / \%$ & 0.0059 & 0.011 & 0.029 & 0.047 & 0.15 & 0.30 \\
\hline SPS1b & $\delta_{\text {SUSY-EW }} / \%$ & $0.0043(1)$ & 0.0050 & 0.027 & 0.021 & -0.064 & -0.21 \\
SPS1b & $\delta_{\text {SUSY-QCD }} / \%$ & 0.0025 & 0.0046 & 0.012 & 0.019 & 0.059 & 0.11 \\
\hline SPS2 & $\delta_{\text {SUSY-EW }} / \%$ & -0.051 & -0.12 & -0.30 & -0.40 & 0.048 & 0.41 \\
SPS2 & $\delta_{\text {SUSY-QCD }} / \%$ & 0.00092 & 0.0017 & 0.0043 & 0.0070 & 0.021 & 0.040 \\
\hline SPS3 & $\delta_{\text {SUSY-EW }} / \%$ & $0.0017(1)$ & 0.0027 & 0.026 & 0.018 & -0.074 & -0.20 \\
SPS3 & $\delta_{\text {SUSY-QCD }} / \%$ & 0.0026 & 0.0048 & 0.012 & 0.020 & 0.061 & 0.12 \\
\hline SPS4 & $\delta_{\text {SUSY-EW }} / \%$ & $0.0076(1)$ & 0.0030 & 0.015 & -0.010 & -0.21 & -0.059 \\
SPS4 & $\delta_{\text {SUSY-QCD }} / \%$ & 0.0034 & 0.0063 & 0.016 & 0.026 & 0.081 & 0.16 \\
\hline SPS5 & $\delta_{\text {SUSY-EW }} / \%$ & 0.020 & 0.024 & 0.13 & 0.14 & 0.037 & $-0.0027(1)$ \\
SPS5 & $\delta_{\text {SUSY-QCD }} / \%$ & 0.0042 & 0.0078 & 0.020 & 0.033 & 0.10 & 0.20 \\
\hline SPS6 & $\delta_{\text {SUSY-EW }} / \%$ & $0.0048(1)$ & 0.0044 & 0.034 & 0.019 & -0.14 & -0.27 \\
SPS6 & $\delta_{\text {SUSY-QCD }} / \%$ & 0.0042 & 0.0078 & 0.020 & 0.033 & 0.10 & 0.20 \\
\hline SPS7 & $\delta_{\text {SUSY-EW }} / \%$ & $0.0054(1)$ & 0.0040 & 0.025 & -0.0017 & -0.21 & -0.30 \\
SPS7 & $\delta_{\text {SUSY-QCD }} / \%$ & 0.0024 & 0.0044 & 0.011 & 0.018 & 0.056 & 0.11 \\
\hline SPS8 & $\delta_{\text {SUSY-EW }} / \%$ & $0.0084(1)$ & 0.0067 & 0.021 & 0.0059 & -0.15 & -0.19 \\
SPS8 & $\delta_{\text {SUSY-QCD }} / \%$ & 0.0017 & 0.0032 & 0.0082 & 0.013 & 0.040 & 0.077 \\
\hline SPS9 & $\delta_{\text {SUSY-EW }} / \%$ & -0.017 & -0.022 & -0.028 & -0.067 & -0.014 & 0.11 \\
SPS9 & $\delta_{\text {SUSY-QCD }} / \%$ & 0.0012 & 0.0022 & 0.0056 & 0.0090 & 0.027 & 0.052 \\
\hline
\end{tabular}

Table 6. Relative SUSY-QCD and SUSY-EW correction factors at the Tevatron for different values of the invariant-mass cut $M_{l l}$ for the SPS benchmark scenarios. The corresponding LO cross sections can be found in table 2 .

scenario where the SUSY-EW corrections reach the $2 \%$ level. The SUSY-QCD corrections reach $1 \%$ for all but the SPS2, SPS8 and SPS9 scenarios but never exceed $2 \%$ for all scenarios in the considered $M_{l l}$ range. 


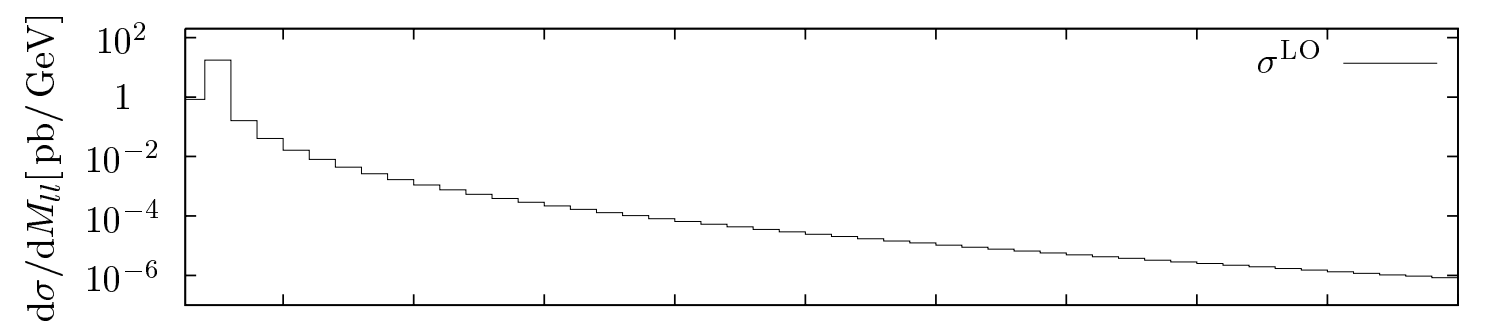

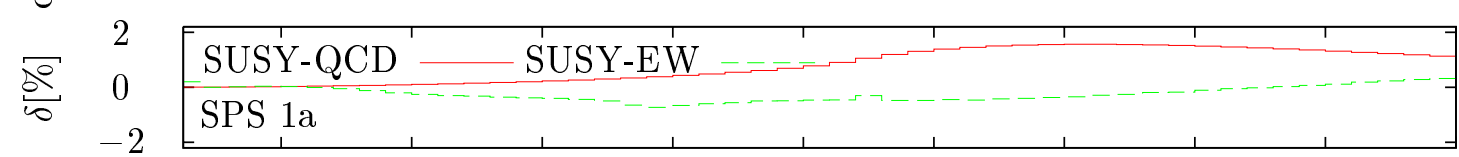

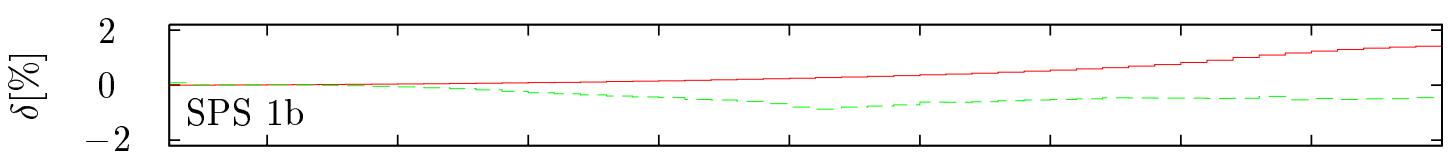

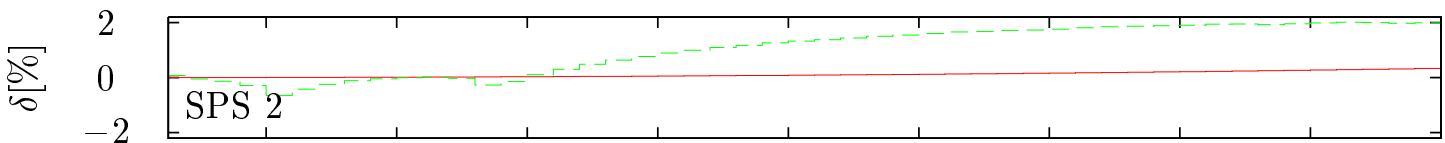
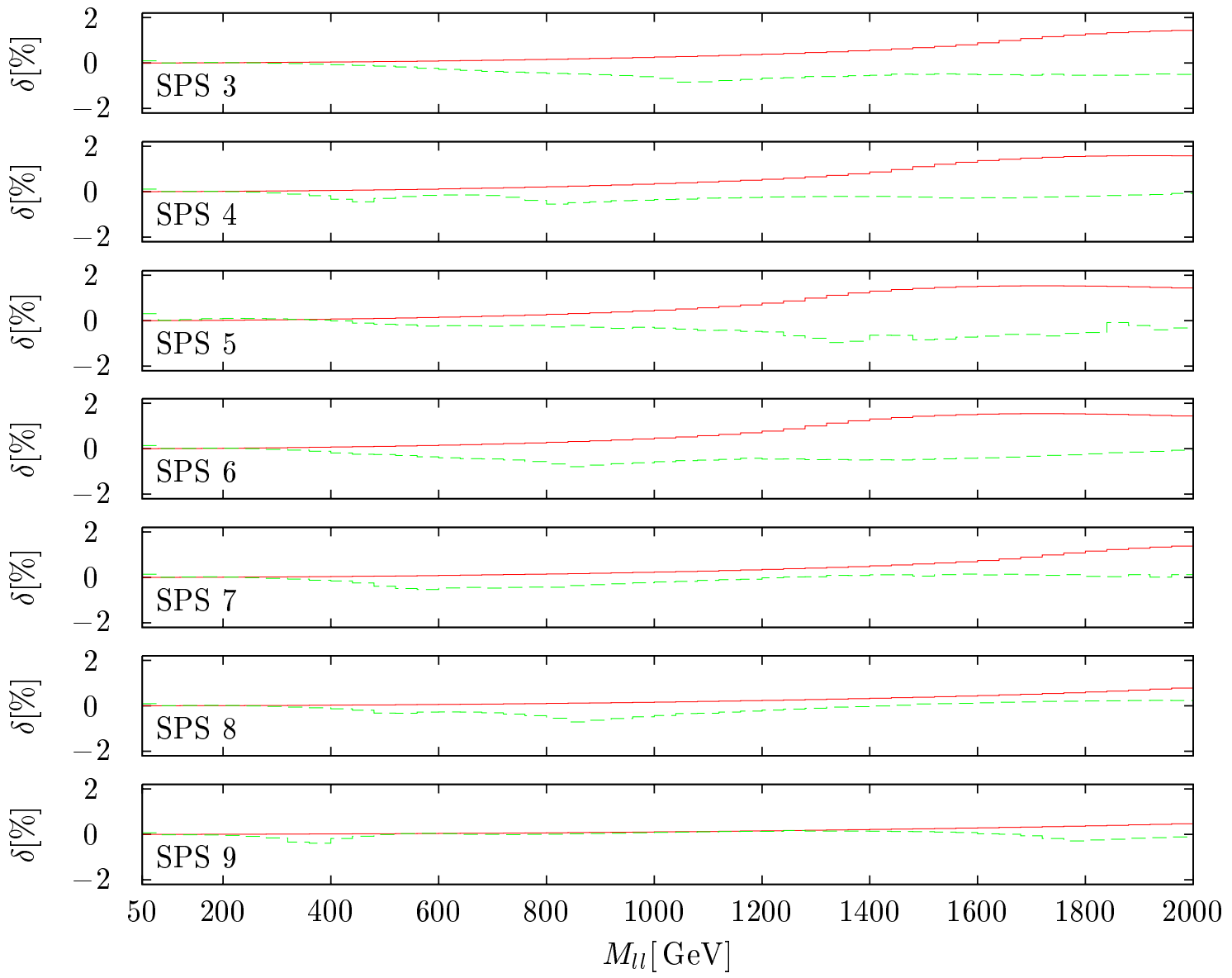

Figure 19. Invariant-dilepton-mass distribution and relative SUSY-QCD and SUSY-EW correction factors at the LHC for the SPS benchmark scenarios. 


\section{Conclusions}

Neutral-current dilepton production represents one of the most important processes at hadron colliders, such as the Tevatron and the LHC. On the one hand, the process acts as a standard candle that is, e.g, indispensable for detector calibration and sensitive to the parton distribution functions of the proton; on the other, it delivers background to many new-physics searches, such as for new $\mathrm{Z}^{\prime}$ bosons. Predictions for this process, thus, ask for the highest possible precision, i.e. both QCD and electroweak corrections have to be included as far as possible.

In this paper we have recalculated and further discussed the next-to-leading order corrections in the Standard Model, where we have compared different methods to include radiative corrections to the Z-boson resonance in a gauge-invariant way. This discussion goes beyond previous work, but our numerical results confirm results existing in the literature. The relevant formulas are listed explicitly and can be used by other groups. We consistently include channels with incoming photons, which starts already with the leadingorder contribution of the $\gamma \gamma \rightarrow l^{-} l^{+}$process. We also include the electroweak corrections to this process and channels induced by $\gamma q / \gamma \bar{q}$ initial states, i.e. all contributions that are formally of electroweak next-to-leading order.

Beyond next-to-leading order we consider multi-photon radiation off the final-state leptons in the structure-function approach, universal electroweak effects, and two-loop electroweak Sudakov logarithms at high energies, so that our predictions are of state-ofthe-art precision in view of electroweak corrections. On the side of the QCD corrections, we include next-to-leading order corrections only, so that further improvements via QCD resummations or interfacing parton showers are desirable.

Our discussion of numerical results comprises a survey of corrections to integrated and differential cross sections, which shows the impact of the various types of corrections in detail. In this context we pay particular attention to effects from incoming photons, because it is not yet clear from results in the literature whether these effects are phenomenologically important or swamped by other uncertainties as, e.g., originating from QCD effects. Our results suggest that effects from $\gamma \gamma$ initial states should be significant in the invariant-mass distribution of the dilepton pair and that these effects can be enhanced to the level of 10-20\% by appropriate cuts. This study is, thus, particularly interesting for a possible future fit of the photon distribution function that is part of the DGLAP evolution in the presence of electroweak corrections.

Finally, we have presented results on radiative corrections within the minimal supersymmetric extension of the Standard Model, which have not yet been known in the literature. Similar to known results on charged-current W-boson production, the difference to the pure Standard Model corrections is small for viable supersymmetry scenarios. In detail, the supersymmetry corrections are below the $0.1 \%$ level and thus irrelevant near the Z-boson resonance and grow only to about $1-2 \%$ in the TeV range for the dilepton invariant mass. These results confirm the role of dilepton production as a standard candle at the Tevatron and the LHC. 


\section{Acknowledgments}

We would like to thank Ansgar Denner, Tobias Kasprzik and Alexander Mück for many interesting and helpful discussions. Moreover, we are grateful to the HORACE team for sending us explicit numbers for the comparison of differential distributions shown in section 5.6. This work is supported in part by the European Community's Marie-Curie Research Training Network HEPTOOLS under contract MRTN-CT-2006-035505.

\section{A Factorization of the photon PDF}

Since the issue of redefining the photon PDF in order to absorb the collinear divergence arising from the splitting $q \rightarrow q \gamma^{*}$ of incoming quarks (or anti-quarks) seems not to be completely settled in the literature yet, we here give a brief account on the analytical structure of this collinear singularity both in dimensional and mass regularization and derive the corresponding factorization formula for the photon PDF in the $\overline{\mathrm{MS}}$ and DIS factorization schemes. To this end, we can make use of existing results, e.g., available in papers on dipole subtraction to deal with this collinear singularity.

In mass regularization this type of singularity is treated in section 5 of ref. [96] in detail. After extracting the singularity from the full phase-space integral by simple subtraction, the singular integration is performed analytically with a small quark mass $m_{q}$ and added back to the result for the cross section. By construction this procedure can be performed with a "spectator" particle in the initial or final state, where its choice is arbitrary for this type of splitting. Writing the (unpolarized) partonic cross-section part that is to be re-added generically as

$$
\sigma_{q a \rightarrow q X}^{\text {sub }}\left(p_{q}, p_{a}\right)=\frac{Q_{q}^{2} \alpha}{2 \pi} \int_{0}^{1} \mathrm{~d} x \mathcal{H}\left(Q^{2}, x\right) \sigma_{\gamma a \rightarrow X}\left(k_{\gamma}=x p_{q}, p_{a}\right),
$$

where $\sigma_{\gamma a \rightarrow X}$ is the partonic cross section of the related process with an incoming photon instead of the $q \rightarrow q \gamma^{*}$ splitting. The momenta $p_{q}$ and $p_{a}$ correspond to the incoming quark $q$ and the other massless particle $a$, respectively, and the functions $\mathcal{H}$ read (cf. eqs. (5.19) and (5.27) of ref. [96])

$$
\begin{aligned}
& \mathcal{H}^{q q, a}(s, x)=\ln \left(\frac{s(1-x)^{2}}{x^{2} m_{q}^{2}}\right) P_{\gamma f}(x)-2 \frac{1-x}{x}, \quad s=\left(p_{q}+p_{a}\right)^{2}, \\
& \mathcal{H}_{j}^{q q}\left(P^{2}, x\right)=\ln \left(\frac{-P^{2}(1-x)}{x^{3} m_{q}^{2}}\right) P_{\gamma f}(x)-2 \frac{1-x}{x}, \quad P^{2}=\left(\tilde{p}_{j}-k_{\gamma}\right)^{2},
\end{aligned}
$$

where $\mathcal{H}^{q q, a}$ refers to the situation with the incoming particle $a$ as spectator and $\mathcal{H}_{j}^{q q}$ to the case with a massless final-state spectator $j$ of momentum $\tilde{p}_{j}$.

The respective results in dimensional regularization can be deduced from the original Catani-Seymour paper [120], where the dipole subtraction method was introduced for massless QCD. The case with an initial-state spectator is described in section 5.5 there, and the case with a final-state spectator in section 5.3. Starting from eq. (5.152) in the 
former case and from eq. (5.73) in the latter, and identifying the QCD coupling factor $C_{\mathrm{F}} \alpha_{\mathrm{S}}$ with the QED factor $Q_{q}^{2} \alpha$, we find

$$
\begin{aligned}
& \mathcal{H}^{q q, a}(s, x)=\frac{1}{\Gamma(1-\epsilon)}\left(\frac{4 \pi \mu^{2}}{s}\right)^{\epsilon}\left[-\frac{1}{\epsilon} P_{\gamma f}(x)+2 P_{\gamma f}(x) \ln (1-x)+x\right], \\
& \mathcal{H}_{j}^{q q}\left(P^{2}, x\right)=\frac{1}{\Gamma(1-\epsilon)}\left(\frac{4 \pi \mu^{2}}{-P^{2}}\right)^{\epsilon}\left[-\frac{1}{\epsilon} P_{\gamma f}(x)+P_{\gamma f}(x) \ln \left(\frac{1-x}{x}\right)+x\right]
\end{aligned}
$$

in $D=4-2 \epsilon$ dimensions. Note that in the last relation we had to translate the kinematical variable $\left(\tilde{p}_{k} p_{a}\right)$ of ref. [120] into our kinematics, which is accomplished by the replacement $\left(\tilde{p}_{k} p_{a}\right) \rightarrow\left(\tilde{p}_{j} p_{q}\right)=\left(\tilde{p}_{j} k_{\gamma}\right) / x=-P^{2} /(2 x)$. Comparing eq. (A.2) with eq. (A.4), or eq. (A.3) with eq. (A.5), we see the following correspondence between the collinear divergence in mass and dimensional regularization,

$$
\frac{\left(4 \pi \mu^{2}\right)^{\epsilon}}{\Gamma(1-\epsilon)} \frac{1}{\epsilon} P_{\gamma f}(x) \leftrightarrow \quad\left(\ln m_{q}^{2}+2 \ln x+1\right) P_{\gamma f}(x)
$$

which is obtained in either case, i.e. with a spectator in the initial or final state, as it should be. The correspondence (A.6) can be used to translate a result for the collinear singularity of the initial-state splitting $q \rightarrow q \gamma^{*}$ from dimensional to mass regularization, or vice versa, in any procedure to treat the collinear divergence, i.e. it is universally valid also in other subtraction procedures or in methods employing phase-space slicing. Applying this correspondence to the photon PDF redefinition in dimensional regularization,

$$
f_{\gamma}^{(h)}(x) \rightarrow f_{\gamma}^{(h)}\left(x, \mu_{\mathrm{F}}^{2}\right)-\frac{\alpha Q_{q}^{2}}{2 \pi} \sum_{a=q, \bar{q}} \int_{x}^{1} \frac{\mathrm{d} z}{z} f_{a}^{(h)}\left(\frac{x}{z}, \mu_{\mathrm{F}}^{2}\right)\left\{\frac{(4 \pi)^{\epsilon}}{\Gamma(1-\epsilon)}\left(\frac{\mu^{2}}{\mu_{\mathrm{F}}^{2}}\right)^{\epsilon} \frac{1}{\epsilon} P_{\gamma f}(z)+C_{\gamma f}^{\mathrm{FS}}(z)\right\}
$$

we obtain the result (3.20) for this redefinition in the mass regularization scheme, where the coefficient function $C_{\gamma f}^{\mathrm{FS}}(z)$ is given by eq. (3.25) for the the two considered factorization schemes (FS), independent of the chosen regularization. Since the $\overline{\mathrm{MS}}$ scheme merely rearranges the divergent terms in dimensional regularization (plus some trivial universal constants), the coefficient function obviously vanishes, $C_{\gamma f}^{\overline{\mathrm{MS}}}(z) \equiv 0$. The fixation of $C_{\gamma f}^{\mathrm{DIS}}(z)$ in the DIS scheme is less trivial. Similar to the gluon PDF redefinition in the DIS scheme for NLO QCD corrections, the choice (3.25) ensures that the whole PDF redefinition does not change the proton momentum (see e.g. eq. (6.9) of ref. [120]).

Finally, we mention a subtle point in the fixation of the coefficient functions in the DIS scheme. The DIS factorization scheme is defined in such a way that the DIS structure function $F_{2}$ from electron-proton scattering does not receive any corrections. This condition uniquely fixes the (anti-)quark PDF redefinition. The gluon PDF redefinition is performed in such a way that the total proton momentum remains unchanged after the PDF redefinition. The simplest choice obviously is to subtract the same $z$-dependent finite part from the gluon PDF that was added to the (anti-)quark PDFs; this is expressed by the analogon of eq. (3.25) in NLO QCD. In NLO QED the role of the gluon is taken over by the photon, which finally leads to eq. (3.25). Obviously, employing the sum rule alone as criterion to fix $C_{\gamma f}^{\mathrm{DIS}}(z)$ is not unique, but involves some convention, since an integral over $z$ 
does not fix the $z$ dependence. A different choice would result in mass regularization if the coefficient functions $C_{a b}^{\mathrm{DIS}}(z)$ were just defined to quantify the finite parts in addition to the divergent contributions that are proportional to $\ln m_{q}^{2} P_{a b}(z)$. The result for the redefined photon PDF would still satisfy the proton momentum sum rule, but the $z$ dependence of the cross section with the incoming photon would be somewhat different. In the first preprint version of this paper such a choice was in fact made, and we found results on the contributions from $q \gamma$ scattering that differ from the results of this paper at the level of up to a few per cent in the off-shell tail of the Z-boson resonance; for observables that are dominated by resonant $\mathrm{Z}$ bosons effects due to $q \gamma$ scattering are negligible in either scheme. The "correct" choice, i.e. the one that is in line with the standard definitions made in NLO QCD, is obtained upon first translating the mass-regularized divergence from the $q \rightarrow q \gamma^{*}$ splitting into dimensional regularization via the correspondence (A.6) and defining the remaining part as coefficient functions, as expressed in eq. (3.20).

\section{B Vertex and box corrections}

In this section we state explicitly the expressions for the vertex form factors $F_{f f V \text {,weak }}^{\sigma}, V=$ $\gamma, \mathrm{Z}$ (3.29), and the photonic (3.7) and weak box diagrams (3.37). The occuring scalar integrals $B_{0}, C_{0}$, and $D_{0}$ depend on their arguments as follows,

$$
\begin{aligned}
& B_{0}\left(p_{1}^{2}, m_{0}, m_{1}\right)=\frac{(2 \pi \mu)^{4-D}}{\mathrm{i} \pi^{2}} \int \mathrm{d}^{D} q \frac{1}{\left[q^{2}-m_{0}^{2}+\mathrm{i} 0\right]\left[\left(q+p_{1}\right)^{2}-m_{1}^{2}+\mathrm{i} 0\right]}, \\
& C_{0}\left(p_{1}^{2},\left(p_{2}-p_{1}\right)^{2}, p_{2}^{2}, m_{0}, m_{1}, m_{2}\right)=\frac{(2 \pi \mu)^{4-D}}{\mathrm{i} \pi^{2}} \int \mathrm{d}^{D} q \\
& \quad \times \frac{1}{\left[q^{2}-m_{0}^{2}+\mathrm{i} 0\right]\left[\left(q+p_{1}\right)^{2}-m_{1}^{2}+\mathrm{i} 0\right]\left[\left(q+p_{2}\right)^{2}-m_{2}^{2}+\mathrm{i} 0\right]}, \\
& D_{0}\left(p_{1}^{2},\left(p_{2}-p_{1}\right)^{2},\left(p_{3}-p_{2}\right)^{2}, p_{3}^{2}, p_{2}^{2},\left(p_{3}-p_{1}\right)^{2}, m_{0}, m_{1}, m_{2}, m_{3}\right)=\frac{(2 \pi \mu)^{4-D}}{\mathrm{i} \pi^{2}} \int \mathrm{d}^{D} q \\
& \quad \times \frac{1}{\left[q^{2}-m_{0}^{2}+\mathrm{i} 0\right]\left[\left(q+p_{1}\right)^{2}-m_{1}^{2}+\mathrm{i} 0\right]\left[\left(q+p_{2}\right)^{2}-m_{2}^{2}+\mathrm{i} 0\right]\left[\left(q+p_{3}\right)^{2}-m_{3}^{2}+\mathrm{i} 0\right]},
\end{aligned}
$$

with $D$ denoting the number of space-time dimensions. The evaluation of these scalar integrals with real or complex masses has been briefly described in section 3 . For the form factors for the weak vertex corrections we obtain

$$
\begin{aligned}
& F_{f f \gamma / \mathrm{Z}, \text { weak }}^{+}(\hat{s})=-\frac{\alpha Q_{f}^{2} s_{\mathrm{W}}^{2}}{4 \pi c_{\mathrm{W}}^{2}}\left\{2-2 \frac{\left(\mu_{\mathrm{Z}}^{2}+2 \hat{s}\right)}{\hat{s}} B_{0}\left(0,0, \mu_{\mathrm{Z}}\right)+\frac{3 \hat{s}+2 \mu_{\mathrm{Z}}^{2}}{\hat{s}} B_{0}(\hat{s}, 0,0)\right. \\
& \left.+\frac{2\left(\mu_{\mathrm{Z}}^{2}+\hat{s}\right)^{2}}{\hat{s}} C_{0}\left(0,0, \hat{s}, 0, \mu_{\mathrm{Z}}, 0\right)\right\}, \\
& F_{f f \gamma, \text { weak }}^{-}(\hat{s})=+\frac{\alpha}{8 \pi}\left\{\frac { 1 } { \hat { s } s _ { \mathrm { W } } ^ { 2 } Q _ { f } } \left[2 Q_{f}\left(2 \hat{s}+\mu_{\mathrm{W}}^{2}\right) B_{0}\left(0,0, \mu_{\mathrm{W}}\right)\right.\right. \\
& +\left(2 I_{\mathrm{w}, f}^{3}-Q_{f}\right)\left(2 \hat{s}+\left(3 \hat{s}+2 \mu_{\mathrm{W}}^{2}\right) B_{0}(\hat{s}, 0,0)\right.
\end{aligned}
$$




$$
\begin{aligned}
& \left.+2\left(\hat{s}+\mu_{\mathrm{W}}^{2}\right)^{2} C_{0}\left(0,0, \hat{s}, 0, \mu_{\mathrm{W}}, 0\right)\right) \\
& -2 I_{\mathrm{w}, f}^{3}\left(\left(\hat{s}+2 \mu_{\mathrm{W}}^{2}\right) B_{0}\left(\hat{s}, \mu_{\mathrm{W}}, \mu_{\mathrm{W}}\right)\right. \\
& \left.\left.-2 \mu_{\mathrm{W}}^{2}\left(2 \hat{s}+\mu_{\mathrm{W}}^{2}\right) C_{0}\left(0,0, \hat{s}, \mu_{\mathrm{W}}, 0, \mu_{\mathrm{W}}\right)\right)\right] \\
& +\frac{\left(I_{\mathrm{w}, f}^{3}-Q_{f} s_{\mathrm{W}}^{2}\right)^{2}}{\hat{s} c_{\mathrm{W}}^{2} s_{\mathrm{W}}^{2}}\left[-4 \hat{s}+4\left(2 \hat{s}+\mu_{\mathrm{Z}}^{2}\right) B_{0}\left(0,0, \mu_{\mathrm{Z}}\right)\right. \\
& -2\left(3 \hat{s}+2 \mu_{\mathrm{Z}}^{2}\right) B_{0}(\hat{s}, 0,0) \\
& \left.\left.-4\left(\hat{s}+\mu_{\mathrm{Z}}^{2}\right)^{2} C_{0}\left(0,0, \hat{s}, 0, \mu_{\mathrm{Z}}, 0\right)\right]\right\} \text {, } \\
& F_{f f \text { Z,weak }}^{-}(\hat{s})=+\frac{\alpha}{8 \pi}\left\{\frac { 1 } { \hat { s } s _ { \mathrm { W } } ^ { 2 } ( I _ { \mathrm { w } , f } ^ { 3 } - Q _ { f } s _ { \mathrm { W } } ^ { 2 } ) } \left[2\left(I_{\mathrm{w}, f}^{3}-Q_{f} s_{\mathrm{W}}^{2}\right)\left(2 \hat{s}+\mu_{\mathrm{W}}^{2}\right) B_{0}\left(0,0, \mu_{\mathrm{W}}\right)\right.\right. \\
& +\left(I_{\mathrm{w}, f}^{3} c_{\mathrm{W}}^{2}-I_{\mathrm{w}, f}^{3} s_{\mathrm{W}}^{2}+Q_{f} s_{\mathrm{W}}^{2}\right)\left(2 \hat{s}+\left(3 \hat{s}+2 \mu_{\mathrm{W}}^{2}\right) B_{0}(\hat{s}, 0,0)\right. \\
& \left.+2\left(\hat{s}+\mu_{\mathrm{W}}^{2}\right)^{2} C_{0}\left(0,0, \hat{s}, 0, \mu_{\mathrm{W}}, 0\right)\right) \\
& -2 c_{\mathrm{W}}^{2} I_{\mathrm{w}, f}^{3}\left(\left(\hat{s}+2 \mu_{\mathrm{W}}^{2}\right) B_{0}\left(\hat{s}, \mu_{\mathrm{W}}, \mu_{\mathrm{W}}\right)\right. \\
& \left.\left.-2 \mu_{\mathrm{W}}^{2}\left(2 \hat{s}+\mu_{\mathrm{W}}^{2}\right) C_{0}\left(0,0, \hat{s}, \mu_{\mathrm{W}}, 0, \mu_{\mathrm{W}}\right)\right)\right] \\
& +\frac{\left(I_{\mathrm{w}, f}^{3}-Q_{f} s_{\mathrm{W}}^{2}\right)^{2}}{\hat{s} c_{\mathrm{W}}^{2} s_{\mathrm{W}}^{2}}\left[-4 \hat{s}+4\left(2 \hat{s}+\mu_{\mathrm{Z}}^{2}\right) B_{0}\left(0,0, \mu_{\mathrm{Z}}\right)\right. \\
& -2\left(3 \hat{s}+2 \mu_{\mathrm{Z}}^{2}\right) B_{0}(\hat{s}, 0,0) \\
& \left.\left.-4\left(\hat{s}+\mu_{\mathrm{Z}}^{2}\right)^{2} C_{0}\left(0,0, \hat{s}, 0, \mu_{\mathrm{Z}}, 0\right)\right]\right\} \text {. }
\end{aligned}
$$

Due to the heavy mass of the top quark the weak corrections for incoming b-quarks differ from those for partonic processes with incoming d/s- quarks by $\delta F_{\mathrm{bb} \gamma / \mathrm{Z} \text {, weak }}^{\sigma}=F_{\mathrm{bb} \gamma / \mathrm{Z} \text {,weak }}^{\sigma}-$ $F_{\mathrm{dd} / \mathrm{ss} \gamma / \mathrm{Z} \text {, weak }}^{\sigma}$, where

$$
\begin{aligned}
\delta F_{\mathrm{bb} \gamma, \text { weak }}^{+}(\hat{s})= & \delta F_{\mathrm{bbZ}, \text { weak }}^{+}(\hat{s})=0 \\
\delta F_{\mathrm{bb} \gamma, \text { weak }}^{-}(\hat{s})=+ & \frac{\alpha}{8 \pi}\left\{-\frac{2}{\hat{s} s_{\mathrm{W}}^{2}}\left[\left(2 \hat{s}+\mu_{\mathrm{W}}^{2}\right)\left(B_{0}\left(0,0, \mu_{\mathrm{W}}\right)-B_{0}\left(0, m_{\mathrm{t}}, \mu_{\mathrm{W}}\right)\right)\right.\right. \\
& +\left(3 \hat{s}+2 \mu_{\mathrm{W}}^{2}\right)\left(B_{0}(\hat{s}, 0,0)-B_{0}\left(\hat{s}, m_{\mathrm{t}}, m_{\mathrm{t}}\right)\right) \\
& +2\left(\hat{s}+\mu_{\mathrm{W}}^{2}\right)^{2}\left(C_{0}\left(0,0, \hat{s}, 0, \mu_{\mathrm{W}}, 0\right)-C_{0}\left(0,0, \hat{s}, m_{\mathrm{t}}, \mu_{\mathrm{W}}, m_{\mathrm{t}}\right)\right) \\
& \left.+3 \mu_{\mathrm{W}}^{2}\left(2 \hat{s}+\mu_{\mathrm{W}}^{2}\right)\left(C_{0}\left(0,0, \hat{s}, \mu_{\mathrm{W}}, 0, \mu_{\mathrm{W}}\right)-C_{0}\left(0,0, \hat{s}, \mu_{\mathrm{W}}, m_{\mathrm{t}}, \mu_{\mathrm{W}}\right)\right)\right] \\
& -\frac{m_{\mathrm{t}}^{2}}{\hat{s} s_{\mathrm{W}}^{2} \mu_{\mathrm{W}}^{2}}\left[\left(m_{\mathrm{t}}^{2}+\mu_{\mathrm{W}}^{2}\right)\right. \\
& \quad \times\left(B_{0}\left(0, m_{\mathrm{t}}, \mu_{\mathrm{W}}\right)+2 B_{0}\left(\hat{s}, m_{\mathrm{t}}, m_{\mathrm{t}}\right)-3 B_{0}\left(\hat{s}, \mu_{\mathrm{W}}, \mu_{\mathrm{W}}\right)\right) \\
& \quad+\hat{s}\left(B_{0}\left(\hat{s}, m_{\mathrm{t}}, m_{\mathrm{t}}\right)-\frac{3}{2} B_{0}\left(\hat{s}, \mu_{\mathrm{W}}, \mu_{\mathrm{W}}\right)-\frac{5}{2}\right)
\end{aligned}
$$




$$
\begin{aligned}
& +\left(\mu_{\mathrm{W}}^{2}\left(2 \hat{s}+3 \mu_{\mathrm{W}}^{2}\right)-m_{\mathrm{t}}^{2}\left(m_{\mathrm{t}}^{2}+\hat{s}\right)\right) \\
& \left.\times\left(2 C_{0}\left(0,0, \hat{s}, m_{\mathrm{t}}, \mu_{\mathrm{W}}, m_{\mathrm{t}}\right)+3 C_{0}\left(0,0, \hat{s}, \mu_{\mathrm{W}}, m_{\mathrm{t}}, \mu_{\mathrm{W}}\right)\right]\right\},(\mathrm{B} \text {. } \\
& \delta F_{\mathrm{bbZ}, \text { weak }}^{-}(\hat{s})=+\frac{\alpha}{8 \pi}\left\{-\frac{1}{\left(2 s_{\mathrm{W}}^{2}-3\right) \hat{s} s_{\mathrm{W}}^{2}}\left[2\left(2 \hat{s}+\mu_{\mathrm{W}}^{2}\right)\left(2 s_{\mathrm{W}}^{2}-3\right)\right.\right. \\
& \times\left(B_{0}\left(0,0, \mu_{\mathrm{W}}\right)-B_{0}\left(0, m_{\mathrm{t}}, \mu_{\mathrm{W}}\right)\right) \\
& +\left(3 \hat{s}+2 \mu_{\mathrm{W}}^{2}\right)\left(4 s_{\mathrm{W}}^{2}-3\right)\left(B_{0}(\hat{s}, 0,0)-B_{0}\left(\hat{s}, m_{\mathrm{t}}, m_{\mathrm{t}}\right)\right) \\
& -12 c_{\mathrm{W}}^{2} \mu_{\mathrm{W}}^{2}\left(2 \hat{s}+\mu_{\mathrm{W}}^{2}\right) \\
& \times\left(C_{0}\left(0,0, \hat{s}, \mu_{\mathrm{W}}, 0, \mu_{\mathrm{W}}\right)-C_{0}\left(0,0, \hat{s}, \mu_{\mathrm{W}}, m_{\mathrm{t}}, \mu_{\mathrm{W}}\right)\right) \\
& +2\left(4 s_{\mathrm{W}}^{2}-3\right)\left(\hat{s}+\mu_{\mathrm{W}}^{2}\right)^{2} \\
& \left.\times\left(C_{0}\left(0,0, \hat{s}, 0, \mu_{\mathrm{W}}, 0\right)-C_{0}\left(0,0, \hat{s}, m_{\mathrm{t}}, \mu_{\mathrm{W}}, m_{\mathrm{t}}\right)\right)\right] \\
& -\frac{m_{\mathrm{t}}^{2}}{\left(2 s_{\mathrm{W}}^{2}-3\right) \hat{s} s_{\mathrm{W}}^{2} \mu_{\mathrm{W}}^{2}}\left[( m _ { \mathrm { t } } ^ { 2 } + \mu _ { \mathrm { W } } ^ { 2 } ) \left(4 B_{0}\left(\hat{s}, m_{\mathrm{t}}, m_{\mathrm{t}}\right) s_{\mathrm{W}}^{2}\right.\right. \\
& -3 B_{0}\left(\hat{s}, \mu_{\mathrm{W}}, \mu_{\mathrm{W}}\right)\left(s_{\mathrm{W}}^{2}-c_{\mathrm{W}}^{2}\right) \\
& \left.+B_{0}\left(0, m_{\mathrm{t}}, \mu_{\mathrm{W}}\right)\left(2 s_{\mathrm{W}}^{2}-3\right)\right)+\left(2 \hat{s} s_{\mathrm{W}}^{2}-6 \mu_{\mathrm{W}}^{2}\right) B_{0}\left(\hat{s}, m_{\mathrm{t}}, m_{\mathrm{t}}\right) \\
& +\hat{s}\left(\frac{3}{2}-5 s_{\mathrm{W}}^{2}\right) \\
& +\left(6 \mu_{\mathrm{W}}^{2}-\frac{3}{2} \hat{s}\left(s_{\mathrm{W}}^{2}-c_{\mathrm{W}}^{2}\right)\right) B_{0}\left(\hat{s}, \mu_{\mathrm{W}}, \mu_{\mathrm{W}}\right) \\
& -12 \hat{s} \mu_{\mathrm{W}}^{2} C_{0}\left(0,0, \hat{s}, m_{\mathrm{t}}, \mu_{\mathrm{W}}, m_{\mathrm{t}}\right) \\
& -3\left(4 \mu_{\mathrm{W}}^{4}-2 m_{\mathrm{t}}^{2} \mu_{\mathrm{W}}^{2}-m_{\mathrm{t}}^{2} \hat{s}\right) \\
& \times\left(C_{0}\left(0,0, \hat{s}, m_{\mathrm{t}}, \mu_{\mathrm{W}}, m_{\mathrm{t}}\right)+C_{0}\left(0,0, \hat{s}, \mu_{\mathrm{W}}, m_{\mathrm{t}}, \mu_{\mathrm{W}}\right)\right) \\
& -3\left(3 \mu_{\mathrm{W}}^{4}-m_{\mathrm{t}}^{4}\right) C_{0}\left(0,0, \hat{s}, \mu_{\mathrm{W}}, m_{\mathrm{t}}, \mu_{\mathrm{W}}\right) \\
& +2 s_{\mathrm{W}}^{2}\left(\mu_{\mathrm{W}}^{2}\left(2 \hat{s}+3 \mu_{\mathrm{W}}^{2}\right)-m_{\mathrm{t}}^{2}\left(m_{\mathrm{t}}^{2}+\hat{s}\right)\right) \\
& \left.\left.\times\left(2 C_{0}\left(0,0, \hat{s}, m_{\mathrm{t}}, \mu_{\mathrm{W}}, m_{\mathrm{t}}\right)+3 C_{0}\left(0,0, \hat{s}, \mu_{\mathrm{W}}, m_{\mathrm{t}}, \mu_{\mathrm{W}}\right)\right)\right]\right\} .
\end{aligned}
$$

As mentioned in section 3.1, the calculation of the box diagrams leads to additional Dirac chains. However, these can be reduced to the Dirac structure (2.5) appearing in the LO matrix element,

$$
\mathcal{A}^{\sigma \tau}=\left[\bar{v}_{q} \gamma^{\mu} \omega_{\sigma} u_{q}\right]\left[\bar{u}_{l} \gamma_{\mu} \omega_{\tau} v_{l}\right]
$$

We used the identities [51]

$$
\begin{aligned}
{\left[\bar{v}_{q} \gamma^{\alpha} \gamma^{\beta} \gamma^{\delta} \omega_{ \pm} u_{q}\right]\left[\bar{u}_{l} \gamma_{\alpha} \gamma^{\beta^{\prime}} \gamma_{\delta} \omega_{ \pm} v_{l}\right] } & =4 g^{\beta \beta^{\prime}} \mathcal{A}^{ \pm \pm} \\
{\left[\bar{v}_{q} \gamma^{\alpha} \gamma^{\beta} \gamma^{\delta} \omega_{ \pm} u_{q}\right]\left[\bar{u}_{l} \gamma_{\alpha} \gamma^{\beta^{\prime}} \gamma_{\delta} \omega_{\mp} v_{l}\right] } & =4\left[\bar{v}_{q} \gamma^{\beta^{\prime}} \omega_{ \pm} u_{q}\right]\left[\bar{u}_{l} \gamma^{\beta} \omega_{\mp} v_{l}\right] \\
{\left[\bar{v}_{q} \not \ell_{1} \omega_{ \pm} u_{q}\right]\left[\bar{u}_{l} \not p_{2} \omega_{ \pm} v_{l}\right] } & =+\hat{u} \mathcal{A}^{ \pm \pm} / 2 \\
{\left[\bar{v}_{q} \not \ell_{1} \omega_{ \pm} u_{q}\right]\left[\bar{u}_{l} \not p_{2} \omega_{\mp} v_{l}\right] } & =-\hat{t} \mathcal{A}^{ \pm \mp} / 2
\end{aligned}
$$


which are valid in four space-time dimensions, to reduce the Dirac structures of the box diagrams. The electroweak corrections due to box diagrams can be written in terms of the functions

$$
\begin{aligned}
& b_{t}^{ \pm \mp}\left(M_{V}, M_{V^{\prime}}, m_{Q}, m_{q}, m_{l}\right)=-2\left\{C_{0}\left(m_{l}^{2}, m_{l}^{2}, \hat{s}, M_{V}, m_{l}, M_{V^{\prime}}\right)+C_{0}\left(m_{q}^{2}, m_{q}^{2}, \hat{s}, M_{V}, m_{Q}, M_{V^{\prime}}\right)\right. \\
& \left.\quad-\left(\hat{t}-m_{Q}^{2}\right) D_{0}\left(m_{q}^{2}, m_{q}^{2}, m_{l}^{2}, m_{l}^{2}, \hat{s}, \hat{t}, M_{V}, m_{Q}, M_{V^{\prime}}, m_{l}\right)\right\} \\
& b_{t}^{ \pm \pm}\left(M_{V}, M_{V^{\prime}}, m_{Q}, m_{q}, m_{l}\right)=\frac{1}{\hat{u}^{2}}\left\{2 \hat{u}\left(B_{0}\left(\hat{s}, M_{V}, M_{V^{\prime}}\right)-B_{0}\left(\hat{t}, m_{Q}, 0\right)\right)\right. \\
& \quad-\hat{t}\left(m_{Q}^{2}-M_{V}^{2}-M_{V^{\prime}}^{2}-\hat{t}+\hat{u}\right)\left(C_{0}\left(m_{l}^{2}, m_{q}^{2}, \hat{t}, 0, M_{V}, m_{Q}\right)+C_{0}\left(m_{l}^{2}, m_{q}^{2}, \hat{t}, 0, M_{V^{\prime}}, m_{Q}\right)\right) \\
& \quad-\left(\hat{t}^{2}+\hat{u}^{2}+\hat{s}\left(m_{Q}^{2}-M_{V}^{2}-M_{V^{\prime}}^{2}\right)\right)\left(C_{0}\left(m_{l}^{2}, m_{l}^{2}, \hat{s}, M_{V}, m_{l}, M_{V^{\prime}}\right)\right. \\
& \left.\quad+C_{0}\left(m_{q}^{2}, m_{q}^{2}, \hat{s}, M_{V}, m_{Q}, M_{V^{\prime}}\right)\right)+\left(\hat{t}\left(M_{V}^{2}+M_{V^{\prime}}^{2}-m_{Q}^{2}-2 \hat{s}\right)^{2}\right. \\
& \left.\quad+\left(\hat{u}\left(2 \hat{u}-m_{Q}^{2}\right)-2 \hat{s}^{2}\right)\left(M_{V}^{2}+M_{V^{\prime}}^{2}-m_{Q}^{2}-2 \hat{s}\right)-2 \hat{u}\left(\hat{u}^{2}-M_{V}^{2} M_{V^{\prime}}^{2}\right)+\hat{u} \hat{s}\left(\hat{s}-m_{Q}^{2}\right)-\hat{s}^{3}\right) \\
& \left.\quad \times D_{0}\left(m_{q}^{2}, m_{q}^{2}, m_{l}^{2}, m_{l}^{2}, \hat{s}, \hat{t}, M_{V}, m_{Q}, M_{V^{\prime}}, m_{l}\right)\right\}, \\
& b_{u}^{ \pm \pm}\left(M_{V}, M_{V^{\prime}}, m_{Q}, m_{q}, m_{l}\right)=-\left.b_{t}^{ \pm \mp}\left(M_{V}, M_{V^{\prime}}, m_{Q}, m_{q}, m_{l}\right)\right|_{\hat{t} \leftrightarrow \hat{u}}, \\
& b_{u}^{ \pm \mp}\left(M_{V}, M_{V^{\prime}}, m_{Q}, m_{q}, m_{l}\right)=-\left.b_{t}^{ \pm \pm}\left(M_{V}, M_{V^{\prime}}, m_{Q}, m_{q}, m_{l}\right)\right|_{\hat{t} \leftrightarrow \hat{u}} .
\end{aligned}
$$

Here the subscripts $q$ and $l$ refer to light quarks and leptons. The heavy mass $m_{Q}$ of the weak isospin partner of down-type quarks is present only in the case of WW box diagrams for incoming b-quarks. In terms of these functions the electroweak box correction factors read

$$
f_{q \bar{q}}^{\mathrm{ZZ}, \sigma \tau}(\hat{s}, \hat{t})=\alpha^{2}\left(g_{q q Z}^{\sigma} g_{l l Z}^{\tau}\right)^{2}\left(b_{t}^{\sigma \tau}\left(\mu_{\mathrm{Z}}, \mu_{\mathrm{Z}}, 0,0,0\right)+b_{u}^{\sigma \tau}\left(\mu_{\mathrm{Z}}, \mu_{\mathrm{Z}}, 0,0,0\right)\right)
$$

for the ZZ box-diagrams, and

$$
\begin{aligned}
f_{q \bar{q}}^{\mathrm{WW},++}(\hat{s}, \hat{t}) & =f_{q \bar{q}}^{\mathrm{WW}, \pm \mp}(\hat{s}, \hat{t})=0, \\
f_{\mathrm{u} \overline{\mathrm{u}}}^{\mathrm{WW},--}(\hat{s}, \hat{t}) & =\frac{\alpha^{2}}{4 s_{\mathrm{W}}^{4}} b_{u}^{--}\left(\mu_{\mathrm{W}}, \mu_{\mathrm{W}}, 0,0,0\right), \\
f_{\mathrm{d} \overline{\mathrm{d}}}^{\mathrm{WW},--}(\hat{s}, \hat{t}) & =\frac{\alpha^{2}}{4 s_{\mathrm{W}}^{4}} b_{t}^{--}\left(\mu_{\mathrm{W}}, \mu_{\mathrm{W}}, 0,0,0\right), \\
f_{\mathrm{b} \overline{\mathrm{b}}}^{\mathrm{WW},--}(\hat{s}, \hat{t}) & =\frac{\alpha^{2}}{4 s_{\mathrm{W}}^{4}} b_{t}^{--}\left(\mu_{\mathrm{W}}, \mu_{\mathrm{W}}, m_{\mathrm{t}}, 0,0\right)
\end{aligned}
$$

for the WW box-diagrams. The photonic box corrections are given by

$$
\begin{aligned}
& f_{q \bar{q}}^{\gamma \gamma, \sigma \tau}=\alpha^{2} Q_{q}^{2} Q_{l}^{2}\left(b_{t}^{\sigma \tau}\left(m_{\gamma}, m_{\gamma}, 0, m_{q}, m_{l}\right)+b_{u}^{\sigma \tau}\left(m_{\gamma}, m_{\gamma}, 0, m_{q}, m_{l}\right)\right), \\
& f_{q \bar{q}}^{Z \gamma, \sigma \tau}=2 \alpha^{2} Q_{q} Q_{l} g_{q q Z}^{\sigma} g_{l l Z}^{\tau}\left(b_{t}^{\sigma \tau}\left(\mu_{\mathrm{Z}}, m_{\gamma}, 0, m_{q}, m_{l}\right)+b_{u}^{\sigma \tau}\left(\mu_{\mathrm{Z}}, m_{\gamma}, 0, m_{q}, m_{l}\right)\right) .
\end{aligned}
$$

\section{SPS benchmark scenarios}

For the SPS benchmark [119] scenarios discussed in this work we use the low-energy input specified in table 7 . The input variables are the ratio $t_{\beta}$ of the vacuum expectation values 
of the Higgs bosons giving rise to up- and down-type fermion masses, the mass of the CP-odd Higgs boson, $M_{\mathrm{A}^{0}}$, the supersymmetric Higgs mass parameter $\mu$, the electroweak gaugino mass parameters $M_{1,2}$, the gluino mass $m_{\tilde{g}}$, the trilinear couplings $A_{\tau, \mathrm{t}, \mathrm{b}}$, the scale at which the $\overline{\mathrm{DR}}$-input values are defined, $\mu_{\mathrm{R}}(\overline{\mathrm{DR}})$, the soft SUSY-breaking parameters in the diagonal entries of the squark and slepton mass matrices of the first and second generations $M_{f i}$ (where $i=L, R$ refers to the left- and right-handed sfermions, $f=q, l$ to quarks and leptons, and $f=\mathrm{u}, \mathrm{d}$, e to up and down quarks and electrons, respectively), and the analogous soft SUSY-breaking parameters for the third generation $M_{f i}^{3 G}$. 


\begin{tabular}{|r|r|r|r|r|r|r|r|r|r|r|}
\hline & SPS 1a & SPS 1b & SPS 2 & SPS 3 & SPS 4 & SPS 5 & SPS 6 & SPS 7 & SPS 8 & SPS 9 \\
\hline$t_{\beta}$ & 10 & 30 & 10 & 10 & 50 & 5 & 10 & 15 & 15 & 10 \\
$M_{\mathrm{A}^{0}}[\mathrm{GeV}]$ & 393.6 & 525.5 & 1443.0 & 572.4 & 404.4 & 693.9 & 463.0 & 377.9 & 514.5 & 911.7 \\
$\mu[\mathrm{GeV}]$ & 352.4 & 495.6 & 124.8 & 508.6 & 377.0 & 639.8 & 393.9 & 300.0 & 398.3 & 869.9 \\
$M_{1}[\mathrm{GeV}]$ & 99.1 & 162.8 & 120.4 & 162.8 & 120.8 & 121.4 & 195.9 & 168.6 & 140.0 & -550.6 \\
$M_{2}[\mathrm{GeV}]$ & 192.7 & 310.9 & 234.1 & 311.4 & 233.2 & 234.6 & 232.1 & 326.8 & 271.8 & -175.5 \\
$m_{\tilde{g}}[\mathrm{GeV}]$ & 595.2 & 916.1 & 784.4 & 914.3 & 721.0 & 710.3 & 708.5 & 926.0 & 820.5 & 1275.2 \\
$A_{\tau}[\mathrm{GeV}]$ & -254.2 & -195.8 & -187.8 & -246.1 & -102.3 & -1179.3 & -213.4 & -39.0 & -36.7 & 1162.4 \\
$A_{\mathrm{t}}[\mathrm{GeV}]$ & -510.0 & -729.3 & -563.7 & -733.5 & -552.2 & -905.6 & -570.0 & -319.4 & -296.7 & -350.3 \\
$A_{\mathrm{b}}[\mathrm{GeV}]$ & -772.7 & -987.4 & -797.2 & -1042.2 & -729.5 & -1671.4 & -811.3 & -350.5 & -330.3 & 216.4 \\
$\mu_{\mathrm{R}}(\overline{\mathrm{DR}})[\mathrm{GeV}]$ & 454.7 & 706.9 & 1077.1 & 703.8 & 571.3 & 449.8 & 548.3 & 839.6 & 987.8 & 1076.1 \\
$M_{q L}[\mathrm{GeV}]$ & 539.9 & 836.2 & 1533.6 & 818.3 & 732.2 & 643.9 & 641.3 & 861.3 & 1081.6 & 1219.2 \\
$M_{\mathrm{d} R}[\mathrm{GeV}]$ & 519.5 & 803.9 & 1530.3 & 788.9 & 713.9 & 622.9 & 621.8 & 828.6 & 1029.0 & 1237.6 \\
$M_{\mathrm{u} R}[\mathrm{GeV}]$ & 521.7 & 807.5 & 1530.5 & 792.6 & 716.0 & 625.4 & 629.3 & 831.3 & 1033.8 & 1227.9 \\
$M_{l L}[\mathrm{GeV}]$ & 196.6 & 334.0 & 1455.6 & 283.3 & 445.9 & 252.2 & 260.7 & 257.2 & 353.5 & 316.2 \\
$M_{e R}[\mathrm{GeV}]$ & 136.2 & 248.3 & 1451.0 & 173.0 & 414.2 & 186.8 & 232.8 & 119.7 & 170.4 & 300.0 \\
$M_{q L}^{3 G}[\mathrm{GeV}]$ & 495.9 & 762.5 & 1295.3 & 760.7 & 640.1 & 535.2 & 591.2 & 836.3 & 1042.7 & 1111.6 \\
$M_{\mathrm{d} R}^{3 G}[\mathrm{GeV}]$ & 516.9 & 780.3 & 1519.9 & 785.6 & 673.4 & 620.5 & 619.0 & 826.9 & 1025.5 & 1231.7 \\
$M_{\mathrm{u} R}^{3 G}[\mathrm{GeV}]$ & 424.8 & 670.7 & 998.5 & 661.2 & 556.8 & 360.5 & 517.0 & 780.1 & 952.7 & 1003.2 \\
$M_{l L}^{3 G}[\mathrm{GeV}]$ & 195.8 & 323.8 & 1449.6 & 282.4 & 394.7 & 250.1 & 259.7 & 256.8 & 352.8 & 307.4 \\
$M_{\mathrm{e} R}^{3 G}[\mathrm{GeV}]$ & 133.6 & 218.6 & 1438.9 & 170.0 & 289.5 & 180.9 & 230.5 & 117.6 & 167.2 & 281.2 \\
\hline
\end{tabular}

Table 7. The low-energy input for the SPS scenarios. See text for details. 
Open Access. This article is distributed under the terms of the Creative Commons Attribution Noncommercial License which permits any noncommercial use, distribution, and reproduction in any medium, provided the original author(s) and source are credited.

\section{References}

[1] TeV4LhC-Top and Electroweak Working Group collaboration, C.E. Gerber et al., Tevatron-for-LHC report: top and electroweak physics, arXiv:0705.3251 [SPIRES].

[2] TeV4LHC Working Group collaboration, S. Abdullin et al., Tevatron-for-LHC report: preparations for discoveries, hep-ph/0608322 [SPIRES].

[3] S. Haywood et al., Electroweak physics, hep-ph/0003275 [SPIRES].

[4] R. Hamberg, W.L. van Neerven and T. Matsuura, A complete calculation of the order $\alpha_{s}^{2}$ correction to the Drell-Yan K factor, Nucl. Phys. B 359 (1991) 343 [Erratum ibid. B 644 (2002) 403] [SPIRES].

[5] W.L. van Neerven and E.B. Zijlstra, The $O\left(\alpha_{s}^{2}\right)$ corrected Drell-Yan $K$ factor in the DIS and MS scheme, Nucl. Phys. B 382 (1992) 11 [Erratum ibid. B 680 (2004) 513] [SPIRES].

[6] R.V. Harlander and W.B. Kilgore, Next-to-next-to-leading order Higgs production at hadron colliders, Phys. Rev. Lett. 88 (2002) 201801 [hep-ph/0201206] [SPIRES].

[7] C. Anastasiou, L.J. Dixon, K. Melnikov and F. Petriello, Dilepton rapidity distribution in the Drell-Yan process at NNLO in QCD, Phys. Rev. Lett. 91 (2003) 182002 [hep-ph/0306192] [SPIRES].

[8] C. Anastasiou, L.J. Dixon, K. Melnikov and F. Petriello, High precision QCD at hadron colliders: electroweak gauge boson rapidity distributions at $N N L O$, Phys. Rev. D 69 (2004) 094008 [hep-ph/0312266] [SPIRES].

[9] K. Melnikov and F. Petriello, The $W$ boson production cross section at the LHC through $O\left(\alpha_{s}^{2}\right)$, Phys. Rev. Lett. 96 (2006) 231803 [hep-ph/0603182] [SPIRES]; Electroweak gauge boson production at hadron colliders through $O\left(\alpha_{s}^{2}\right)$, Phys. Rev. D 74 (2006) 114017 [hep-ph/0609070] [SPIRES].

[10] S. Catani, L. Cieri, G. Ferrera, D. de Florian and M. Grazzini, Vector boson production at hadron colliders: a fully exclusive QCD calculation at NNLO,

Phys. Rev. Lett. 103 (2009) 082001 [arXiv:0903.2120] [SPIRES].

[11] S. Moch and A. Vogt, Higher-order soft corrections to lepton pair and Higgs boson production, Phys. Lett. B 631 (2005) 48 [hep-ph/0508265] [SPIRES].

[12] E. Laenen and L. Magnea, Threshold resummation for electroweak annihilation from DIS data, Phys. Lett. B 632 (2006) 270 [hep-ph/0508284] [SPIRES].

[13] A. Idilbi, X.-D. Ji, J.-P. Ma and F. Yuan, Threshold resummation for Higgs production in effective field theory, Phys. Rev. D 73 (2006) 077501 [hep-ph/0509294] [SPIRES].

[14] V. Ravindran and J. Smith, Threshold corrections to rapidity distributions of $Z$ and $W^{ \pm}$ bosons beyond $N^{2}$ LO at hadron colliders, Phys. Rev. D 76 (2007) 114004 [arXiv:0708.1689] [SPIRES].

[15] S. Frixione and B.R. Webber, The MCNLO 3.3 event generator, hep-ph/0612272 [SPIRES]. 
[16] P.B. Arnold and R.P. Kauffman, $W$ and $Z$ production at next-to-leading order: from large q(t) to small, Nucl. Phys. B 349 (1991) 381 [SPIRES].

[17] C. Balázs, J.-W. Qiu and C.P. Yuan, Effects of QCD resummation on distributions of leptons from the decay of electroweak vector bosons, Phys. Lett. B 355 (1995) 548 [hep-ph/9505203] [SPIRES].

[18] C. Balázs and C.P. Yuan, Soft gluon effects on lepton pairs at hadron colliders, Phys. Rev. D 56 (1997) 5558 [hep-ph/9704258] [SPIRES].

[19] R.K. Ellis, D.A. Ross and S. Veseli, Vector boson production in hadronic collisions, Nucl. Phys. B 503 (1997) 309 [hep-ph/9704239] [SPIRES].

[20] R.K. Ellis and S. Veseli, $W$ and $Z$ transverse momentum distributions: resummation in $q_{T}$ space, Nucl. Phys. B 511 (1998) 649 [hep-ph/9706526] [SPIRES].

[21] J.-W. Qiu and X.-F. Zhang, QCD prediction for heavy boson transverse momentum distributions, Phys. Rev. Lett. 86 (2001) 2724 [hep-ph/0012058] [SPIRES]; Role of the nonperturbative input in QCD resummed Drell-Yan $q_{T}$ distributions, Phys. Rev. D 63 (2001) 114011 [hep-ph/0012348] [SPIRES].

[22] A. Kulesza and W.J. Stirling, Soft gluon resummation in transverse momentum space for electroweak boson production at hadron colliders, Eur. Phys. J. C 20 (2001) 349 [hep-ph/0103089] [SPIRES].

[23] A. Kulesza, G. Sterman and W. Vogelsang, Joint resummation in electroweak boson production, Phys. Rev. D 66 (2002) 014011 [hep-ph/0202251] [SPIRES].

[24] F. Landry, R. Brock, P.M. Nadolsky and C.P. Yuan, Tevatron Run-1 Z boson data and Collins-Soper-Sterman resummation formalism, Phys. Rev. D 67 (2003) 073016 [hep-ph/0212159] [SPIRES].

[25] S. Berge, P.M. Nadolsky and F.I. Olness, Heavy-flavor effects in soft gluon resummation for electroweak boson production at hadron colliders, Phys. Rev. D 73 (2006) 013002 [hep-ph/0509023] [SPIRES].

[26] G. Bozzi, S. Catani, G. Ferrera, D. de Florian and M. Grazzini, Transverse-momentum resummation: a perturbative study of $Z$ production at the Tevatron, Nucl. Phys. B $\mathbf{8 1 5}$ (2009) 174 [arXiv:0812.2862] [SPIRES].

[27] U. Baur, S. Keller and D. Wackeroth, Electroweak radiative corrections to $W$ boson production in hadronic collisions, Phys. Rev. D 59 (1999) 013002 [hep-ph/9807417] [SPIRES].

[28] V.A. Zykunov, Electroweak corrections to the observables of W-boson production at RHIC, Eur. Phys. J. Direct C 3 (2001) 9 [hep-ph/0107059] [SPIRES].

[29] U. Baur and D. Wackeroth, Electroweak radiative corrections to $p \bar{p} \rightarrow W^{ \pm} \rightarrow \ell^{ \pm} \nu$ beyond the pole approximation, Phys. Rev. D 70 (2004) 073015 [hep-ph/0405191] [SPIRES].

[30] A. Arbuzov et al., One-loop corrections to the Drell-Yan process in SANC. I: the charged current case, Eur. Phys. J. C 46 (2006) 407 [Erratum ibid. C 50 (2007) 505] [hep-ph/0506110] [SPIRES].

[31] S. Dittmaier and M. Krämer, Electroweak radiative corrections to W-boson production at hadron colliders, Phys. Rev. D 65 (2002) 073007 [hep-ph/0109062] [SPIRES]. 
[32] C.M. Carloni Calame, G. Montagna, O. Nicrosini and A. Vicini, Precision electroweak calculation of the charged current Drell-Yan process, JHEP 12 (2006) 016 [hep-ph/0609170] [SPIRES].

[33] U. Baur, S. Keller and W.K. Sakumoto, QED radiative corrections to $Z$ boson production and the forward backward asymmetry at hadron colliders, Phys. Rev. D 57 (1998) 199 [hep-ph/9707301] [SPIRES].

[34] U. Baur, O. Brein, W. Hollik, C. Schappacher and D. Wackeroth, Electroweak radiative corrections to neutral current Drell-Yan processes at hadron colliders, Phys. Rev. D 65 (2002) 033007 [hep-ph/0108274] [SPIRES].

[35] V.A. Zykunov, Weak radiative corrections to the Drell-Yan process for large invariant mass of a dilepton pair, Phys. Rev. D 75 (2007) 073019 [hep-ph/0509315] [SPIRES].

[36] C.M. Carloni Calame, G. Montagna, O. Nicrosini and A. Vicini, Precision electroweak calculation of the production of a high transverse-momentum lepton pair at hadron colliders, JHEP 10 (2007) 109 [arXiv:0710.1722] [SPIRES].

[37] A. Arbuzov et al., One-loop corrections to the Drell-Yan process in SANC. (II). The neutral current case, Eur. Phys. J. C 54 (2008) 451 [arXiv:0711.0625] [SPIRES].

[38] C. Buttar et al., Les Houches physics at TeV colliders 2005, Standard Model and Higgs working group: summary report, hep-ph/0604120 [SPIRES].

[39] C. Buttar et al., Standard Model handles and Candles working group: tools and jets summary report, arXiv: 0803.0678 [SPIRES].

[40] S. Dittmaier and M. Krämer, Tuned comparison of electroweak corrections to Drell-Yan-like $W$ - and Z-boson production - a status report, in Les Houches physics at TeV colliders 2005, Standard Model and Higgs working group: summary report, section 10 hep-ph/0604120 [SPIRES].

[41] A.B. Arbuzov and R.R. Sadykov, Inverse Bremsstrahlung contributions to Drell-Yan like processes, J. Exp. Theor. Phys. 106 (2008) 488 [arXiv:0707.0423] [SPIRES].

[42] S. Brensing, S. Dittmaier, M. Krämer and A. Mück, Radiative corrections to $W^{-}$boson hadroproduction: higher-order electroweak and supersymmetric effects, Phys. Rev. D 77 (2008) 073006 [arXiv:0710.3309] [SPIRES].

[43] W. Placzek and S. Jadach, Multiphoton radiation in leptonic W-boson decays, Eur. Phys. J. C 29 (2003) 325 [hep-ph/0302065] [SPIRES].

[44] C.M. Carloni Calame, G. Montagna, O. Nicrosini and M. Treccani, Higher-order QED corrections to $W$-boson mass determination at hadron colliders, Phys. Rev. D 69 (2004) 037301 [hep-ph/0303102] [SPIRES].

[45] C.M. Carloni Calame, S. Jadach, G. Montagna, O. Nicrosini and W. Placzek, Comparisons of the Monte Carlo programs HORACE and WINHAC for single W-boson production at hadron colliders, Acta Phys. Polon. B 35 (2004) 1643 [hep-ph/0402235] [SPIRES].

[46] C.M. Carloni Calame, G. Montagna, O. Nicrosini and M. Treccani, Multiple photon corrections to the neutral-current Drell-Yan process, JHEP 05 (2005) 019 [hep-ph/0502218] [SPIRES].

[47] G. Balossini et al., Electroweak \&3 QCD corrections to Drell-Yan processes, Acta Phys. Polon. B 39 (2008) 1675 [arXiv:0805.1129] [SPIRES]. 
[48] G. Balossini et al., Combination of electroweak and QCD corrections to single $W$ production at the Fermilab Tevatron and the CERN LHC, arXiv:0907.0276 [SPIRES].

[49] A. Kotikov, J.H. Kühn and O. Veretin, Two-loop formfactors in theories with mass gap and Z-boson production, Nucl. Phys. B 788 (2008) 47 [hep-ph/0703013] [SPIRES].

[50] A. Denner, S. Dittmaier, M. Roth and D. Wackeroth, Predictions for all processes $e^{+} e^{-} \rightarrow 4$ fermions $+\gamma$, Nucl. Phys. B 560 (1999) 33 [hep-ph/9904472] [SPIRES].

[51] A. Denner, S. Dittmaier, M. Roth and L.H. Wieders, Electroweak corrections to charged-current $e^{+} e^{-} \rightarrow 4$ fermion processes: technical details and further results, Nucl. Phys. B 724 (2005) 247 [hep-ph/0505042] [SPIRES].

[52] R.G. Stuart, Gauge invariance, analyticity and physical observables at the $Z^{0}$ resonance, Phys. Lett. B 262 (1991) 113 [SPIRES].

[53] A. Aeppli, F. Cuypers and G.J. van Oldenborgh, $O(\gamma)$ corrections to $W$ pair production in $e^{+} e^{-}$and $\gamma \gamma$ collisions, Phys. Lett. B 314 (1993) 413 [hep-ph/9303236] [SPIRES].

[54] A. Aeppli, G.J. van Oldenborgh and D. Wyler, Unstable particles in one loop calculations, Nucl. Phys. B 428 (1994) 126 [hep-ph/9312212] [SPIRES].

[55] H.G.J. Veltman, Mass and width of unstable gauge bosons, Z. Phys. C 62 (1994) 35 [SPIRES].

[56] A. Denner and S. Dittmaier, Production of light fermion antifermion pairs in $\gamma \gamma$ collisions, Eur. Phys. J. C 9 (1999) 425 [hep-ph/9812411] [SPIRES].

[57] E.A. Kuraev and V.S. Fadin, On radiative corrections to $e^{+} e^{-}$single photon annihilation at high-energy, Sov. J. Nucl. Phys. 41 (1985) 466 [Yad. Fiz. 41 (1985) 733] [SPIRES].

[58] G. Altarelli and G. Martinelli, Radiative corrections to the $Z^{0}$ line shape at LEP, in Physics at LEP, volume 1, J. Ellis and R.D. Peccei eds., (1986) pg. 47 [SPIRES].

[59] O. Nicrosini and L. Trentadue, Soft photons and second order radiative corrections to $e^{+} e^{-} \rightarrow Z^{0}$, Phys. Lett. B 196 (1987) 551 [SPIRES].

[60] O. Nicrosini and L. Trentadue, Second order electromagnetic radiative corrections to $e^{+} e^{-} \rightarrow \gamma^{*}, Z^{0} \rightarrow \mu^{+} \mu^{-}$, Z. Phys. C 39 (1988) 479 [SPIRES].

[61] F.A. Berends, W.L. van Neerven and G.J.H. Burgers, Higher order radiative corrections at LEP energies, Nucl. Phys. B 297 (1988) 429 [Erratum ibid. B 304 (1988) 921] [SPIRES].

[62] A.B. Arbuzov, Non-singlet splitting functions in QED, Phys. Lett. B 470 (1999) 252 [hep-ph/9908361] [SPIRES].

[63] A. Denner and S. Dittmaier, The complex-mass scheme for perturbative calculations with unstable particles, Nucl. Phys. (Proc. Suppl.) 160 (2006) 22 [hep-ph/0605312] [SPIRES].

[64] M.W. Grunewald et al., Four-fermion production in electron positron collisions, in Reports of the working groups on precision calculations for LEP2 physics,S. Jadach, G. Passarino and R. Pittau eds., CERN 2000-009, Geneva Switzerland (2000), pg. 1 [hep-ph/0005309] [SPIRES].

[65] S. Dittmaier and A. Kaiser, Photonic and QCD radiative corrections to Higgs boson production in $\mu^{+} \mu^{-} \rightarrow f \bar{f}$, Phys. Rev. D 65 (2002) 113003 [hep-ph/0203120] [SPIRES].

[66] A. Denner, Techniques for calculation of electroweak radiative corrections at the one loop level and results for W physics at LEP-200, Fortschr. Phys. 41 (1993) 307 [arXiv:0709.1075] [SPIRES]. 
[67] D.Y. Bardin, A. Leike, T. Riemann and M. Sachwitz, Energy dependent width effects in $e^{+} e^{-}$annihilation near the $Z$ pole, Phys. Lett. B 206 (1988) 539 [SPIRES].

[68] W. Beenakker et al., The fermion loop scheme for finite width effects in $e^{+} e^{-}$annihilation into four fermions, Nucl. Phys. B 500 (1997) 255 [hep-ph/9612260] [SPIRES].

[69] A. Sirlin, Theoretical considerations concerning the $Z^{0}$ mass, Phys. Rev. Lett. 67 (1991) 2127 [SPIRES].

[70] A. Sirlin, Observations concerning mass renormalization in the electroweak theory, Phys. Lett. B 267 (1991) 240 [SPIRES].

[71] R.G. Stuart, The structure of the $Z^{0}$ resonance and the physical properties of the $Z^{0}$ boson, Phys. Rev. Lett. 70 (1993) 3193 [SPIRES].

[72] P. Gambino and P.A. Grassi, The Nielsen identities of the SM and the definition of mass, Phys. Rev. D 62 (2000) 076002 [hep-ph/9907254] [SPIRES].

[73] P.A. Grassi, B.A. Kniehl and A. Sirlin, Width and partial widths of unstable particles in the light of the Nielsen identities, Phys. Rev. D 65 (2002) 085001 [hep-ph/0109228] [SPIRES].

[74] A. Sirlin, Radiative corrections in the $\mathrm{SU}(2)_{L} \times \mathrm{U}(1)$ theory: a simple renormalization framework, Phys. Rev. D 22 (1980) 971 [SPIRES].

[75] W.J. Marciano and A. Sirlin, Radiative corrections to neutrino induced neutral current phenomena in the $\mathrm{SU}(2)_{L} \times \mathrm{U}(1)$ theory, Phys. Rev. D 22 (1980) 2695 [Erratum ibid. D 31 (1980) 213] [SPIRES].

[76] A. Sirlin and W.J. Marciano, Radiative corrections to muon-neutrino $N \rightarrow \mu^{-} X$ and their effect on the determination of $\rho^{2}$ and $\sin ^{2} \theta_{W}$, Nucl. Phys. B 189 (1981) 442 [SPIRES].

[77] G. Altarelli, R. Kleiss and C. Verzegnassi eds., Z physics at LEP1, volume 1, CERN 89-08, Geneva Switzerland (1989).

[78] D.Y. Bardin, M. Grunewald and G. Passarino, Precision calculation project report, hep-ph/9902452 [SPIRES].

[79] J. Küblbeck, M. Böhm and A. Denner, FeynArts: computer algebraic generation of Feynman graphs and amplitudes, Comput. Phys. Commun. 60 (1990) 165 [SPIRES].

[80] H. Eck and J. Küblbeck, Guide to FeynArts 1.0, University of Würzburg, Würzburg Germany (1992).

[81] T. Hahn, Generating Feynman diagrams and amplitudes with FeynArts 3, Comput. Phys. Commun. 140 (2001) 418 [hep-ph/0012260] [SPIRES].

[82] T. Hahn and M. Pérez-Victoria, Automatized one-loop calculations in four and D dimensions, Comput. Phys. Commun. 118 (1999) 153 [hep-ph/9807565] [SPIRES].

[83] T. Hahn, Automatic loop calculations with FeynArts, FormCalc and LoopTools, Nucl. Phys. (Proc. Suppl.) 89 (2000) 231 [hep-ph/0005029] [SPIRES].

[84] R. Mertig, M. Böhm and A. Denner, FEYN CALC: computer algebraic calculation of Feynman amplitudes, Comput. Phys. Commun. 64 (1991) 345 [SPIRES].

[85] R. Mertig, Guide to FeynCalc 1.0, University of Würzburg, Würzburg Germany (1992).

[86] G. Passarino and M.J.G. Veltman, One loop corrections for $e^{+} e^{-}$annihilation into $\mu^{+} \mu^{-}$in the Weinberg model, Nucl. Phys. B 160 (1979) 151 [SPIRES]. 
[87] G. 't Hooft and M.J.G. Veltman, Scalar one loop integrals, Nucl. Phys. B 153 (1979) 365 [SPIRES].

[88] W. Beenakker and A. Denner, Infrared divergent scalar box integrals with applications in the electroweak Standard Model, Nucl. Phys. B 338 (1990) 349 [SPIRES].

[89] A. Denner, U. Nierste and R. Scharf, A compact expression for the scalar one loop four point function, Nucl. Phys. B 367 (1991) 637 [SPIRES].

[90] A. Denner and S. Dittmaier, Reduction schemes for one-loop tensor integrals, Nucl. Phys. B 734 (2006) 62 [hep-ph/0509141] [SPIRES].

[91] A. Denner and S. Dittmaier, The scalar four-point function, in preparation.

[92] S. Dittmaier, Separation of soft and collinear singularities from one-loop N-point integrals, Nucl. Phys. B 675 (2003) 447 [hep-ph/0308246] [SPIRES].

[93] A. Bredenstein, A. Denner, S. Dittmaier and S. Pozzorini, NLO QCD corrections to top anti-top bottom anti-bottom production at the LHC: 1. quark-antiquark annihilation, JHEP 08 (2008) 108 [arXiv:0807.1248] [SPIRES].

[94] S. Dittmaier, Weyl-van-der-Waerden formalism for helicity amplitudes of massive particles, Phys. Rev. D 59 (1999) 016007 [hep-ph/9805445] [SPIRES].

[95] S. Dittmaier, A general approach to photon radiation off fermions, Nucl. Phys. B 565 (2000) 69 [hep-ph/9904440] [SPIRES].

[96] S. Dittmaier, A. Kabelschacht and T. Kasprzik, Polarized QED splittings of massive fermions and dipole subtraction for non-collinear-safe observables, Nucl. Phys. B 800 (2008) 146 [arXiv:0802.1405] [SPIRES].

[97] K.P.O. Diener, S. Dittmaier and W. Hollik, Electroweak higher-order effects and theoretical uncertainties in deep-inelastic neutrino scattering, Phys. Rev. D 72 (2005) 093002 [hep-ph/0509084] [SPIRES].

[98] A.D. Martin, R.G. Roberts, W.J. Stirling and R.S. Thorne, Parton distributions incorporating QED contributions, Eur. Phys. J. C 39 (2005) 155 [hep-ph/0411040] [SPIRES].

[99] H. Burkhardt and B. Pietrzyk, Update of the hadronic contribution to the QED vacuum polarization, Phys. Lett. B 356 (1995) 398 [SPIRES].

[100] S. Eidelman and F. Jegerlehner, Hadronic contributions to $g-2$ of the leptons and to the effective fine structure constant $\alpha\left(M_{z}^{2}\right), Z$. Phys. C 67 (1995) 585 [hep-ph/9502298] [SPIRES].

[101] M. Consoli, W. Hollik and F. Jegerlehner, Electroweak radiative corrections for Z physics, presented at Workshop on Z Physics at LEP, CERN-TH-5527-89, Cern, Geneva Switzerland (1989) [SPIRES].

[102] M. Consoli, W. Hollik and F. Jegerlehner, The effect of the top quark on the $M(W)-M(Z)$ interdependence and possible decoupling of heavy fermions from low-energy physics, Phys. Lett. B 227 (1989) 167 [SPIRES].

[103] J. Fleischer, O.V. Tarasov and F. Jegerlehner, Two loop heavy top corrections to the rho parameter: A Simple formula valid for arbitrary Higgs mass, Phys. Lett. B 319 (1993) 249 [SPIRES]. 
[104] V.S. Fadin, L.N. Lipatov, A.D. Martin and M. Melles, Resummation of double logarithms in electroweak high energy processes, Phys. Rev. D 61 (2000) 094002 [hep-ph/9910338] [SPIRES].

[105] M. Ciafaloni, P. Ciafaloni and D. Comelli, Bloch-Nordsieck violating electroweak corrections to inclusive TeV scale hard processes, Phys. Rev. Lett. 84 (2000) 4810 [hep-ph/0001142] [SPIRES].

[106] M. Hori, H. Kawamura and J. Kodaira, Electroweak Sudakov at two loop level, Phys. Lett. B 491 (2000) 275 [hep-ph/0007329] [SPIRES].

[107] M. Melles, Resummation of angular dependent corrections in spontaneously broken gauge theories, Eur. Phys. J. C 24 (2002) 193 [hep-ph/0108221] [SPIRES].

[108] W. Beenakker and A. Werthenbach, Electroweak two-loop Sudakov logarithms for on-shell fermions and bosons, Nucl. Phys. B 630 (2002) 3 [hep-ph/0112030] [SPIRES].

[109] A. Denner, M. Melles and S. Pozzorini, Two-loop electroweak angular-dependent logarithms at high energies, Nucl. Phys. B 662 (2003) 299 [hep-ph/0301241] [SPIRES].

[110] B. Jantzen, J.H. Kühn, A.A. Penin and V.A. Smirnov, Two-loop electroweak logarithms, Phys. Rev. D 72 (2005) 051301 [Erratum ibid. D 74 (2006) 019901] [hep-ph/0504111] [SPIRES].

[111] B. Jantzen, J.H. Kühn, A.A. Penin and V.A. Smirnov, Two-loop electroweak logarithms in four-fermion processes at high energy, Nucl. Phys. B 731 (2005) 188 [Erratum ibid. B 752 (2006) 327] [hep-ph/0509157] [SPIRES].

[112] A. Denner, B. Jantzen and S. Pozzorini, Two-loop electroweak next-to-leading logarithmic corrections to massless fermionic processes, Nucl. Phys. B 761 (2007) 1 [hep-ph/0608326] [SPIRES].

[113] P. Ciafaloni and D. Comelli, The importance of weak bosons emission at LHC, JHEP 09 (2006) 055 [hep-ph/0604070] [SPIRES].

[114] U. Baur, Weak boson emission in hadron collider processes, Phys. Rev. D 75 (2007) 013005 [hep-ph/0611241] [SPIRES].

[115] T. Kinoshita, Mass singularities of Feynman amplitudes, J. Math. Phys. 3 (1962) 650 [SPIRES].

[116] T.D. Lee and M. Nauenberg, Degenerate systems and mass singularities, Phys. Rev. 133 (1964) B1549 [SPIRES].

[117] Particle Data Group collaboration, W.M. Yao et al., Review of particle physics, J. Phys. G 33 (2006) 1 [SPIRES].

[118] F. Jegerlehner, The effective fine structure constant at TESLA energies, DESY 01-029 [LC-TH-2001-035] [hep-ph/0105283] [SPIRES].

[119] B.C. Allanach et al., The Snowmass points and slopes: benchmarks for SUSY searches, Eur. Phys. J. C 25 (2002) 113 [eConf C 010630 (2001) P125] [hep-ph/0202233] [SPIRES].

[120] S. Catani and M.H. Seymour, A general algorithm for calculating jet cross sections in NLO QCD, Nucl. Phys. B 485 (1997) 291 [Erratum ibid. B 510 (1998) 503] [hep-ph/9605323] [SPIRES]. 